\title{
Kinematic Calibration of a Serial Robotic Arm Using a Linear Movement Constraint
}

\author{
Katie DiCola, B. Eng. \\ A thesis submitted to the \\ Faculty of Graduate Studies and Research \\ in partial fulfillment of the requirements for the degree of
}

Master of Applied Science in Mechanical Engineering

Ottawa-Carleton Institute for Mechanical and Aerospace Engineering

Department of Mechanical and Aerospace Engineering

Carleton University

Ottawa, Ontario

August 12, 2016

(C) Copyright

Katie DiCola, 2016 
The undersigned hereby recommends to the

Faculty of Graduate Studies and Research acceptance of the thesis

\title{
Kinematic Calibration of a Serial Robotic Arm Using a Linear Movement Constraint
}

\author{
submitted by Katie DiCola, B. Eng. \\ in partial fulfillment of the requirements for the degree of \\ Master of Applied Science in Mechanical Engineering
}

Professor M. J. D. Hayes, Thesis Supervisor

Professor Metin Yaras, Chair,

Department of Mechanical and Aerospace Engineering

Ottawa-Carleton Institute for Mechanical and Aerospace Engineering

Department of Mechanical and Aerospace Engineering

Carleton University

January, 2016 


\begin{abstract}
Kinematic calibration of a robot arm is necessary for the performance of many tasks. The calibration process is frequently a tradeoff between affordability and accuracy. In this thesis, a method is developed to calibrate a robotic arm using relatively inexpensive optical sensors. A simulation of the calibration process is developed that shows accurate determination of angular offsets in the robot model is possible. These offsets account for the majority of error in robot movement. This method was performed on two industrial robotic arms: a Fanuc S-420iF and a Motoman MH-180. The joint angle offsets were determined in each case, though accuracy was lower in practice than in simulation. The method was deemed to be potentially viable for identifying joint angle offsets. With some modifications, it may also be viable for identifying link twist offsets.
\end{abstract}




\section{Acknowledgments}

I would like to thank my supervisor, Prof. John Hayes, for his help and patience with all the work I've done on my thesis. He has been instrumental in all the work I've done so far, and in giving me the opportunity to work on this project in the first place. I'd also like to thank everyone at EDI (Enclosures Direct Inc.) for allowing me to spend so much time in their offices and factory floor, working with the robots. I'd particularly like to thank Steve Christie for the opportunity to work with these robots, and Jim Robinson, who helped me figure out how to use them.

I'd also like to thank the staff and faculty at Carleton, specifically the Mechanical and Aerospace Engineering department, for the support and opportunities I received both in my undergraduate and graduate studies.

Lastly I would like to thank my parents for the fantastic education I've received, and for always encouraging me to continue with it. 


\section{Table of Contents}

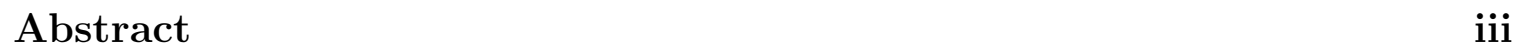

Acknowledgments iv

Table of Contents $\quad \mathrm{v}$

$\begin{array}{ll}\text { List of Tables } & \text { ix }\end{array}$

List of Figures $\quad x$

\begin{tabular}{lll}
\hline & Introduction & 1
\end{tabular}

1.1 Motivation . . . . . . . . . . . . . . . . . . . . 1

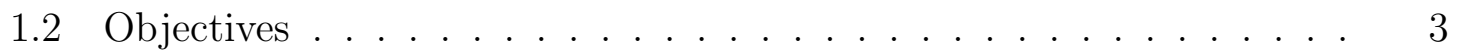

1.3 Statement of Originality $\ldots \ldots \ldots \ldots \ldots$

1.4 Thesis Overview . . . . . . . . . . . . . . . . . . . . . . . 4

\begin{tabular}{|lll}
\hline 2 & Theory and Literature Review & 6
\end{tabular}

$2.1 \quad$ Manipulator Kinematics $\ldots \ldots \ldots \ldots \ldots$

2.1 .1 Overview of Manipulator Kinematics . . . . . . . . . . 6

2.1 .2 Types of Robotic Manipulators $\ldots \ldots \ldots \ldots$

2.1 .3 Joints . . . . . . . . . . . . . . . . . . . . . . 11

2.1.4 Attachment of Reference Frames to Links . . . . . . . . . 13

2.1 .5 Denavit-Hartenberg Parameters . . . . . . . . . . . . . 13

2.1 .6 Variation in DH Parametrizations . . . . . . . . . . . . . 16

2.1 .7 Forward Kinematics $\ldots \ldots \ldots$

2.1 .8 Inverse Kinematics . . . . . . . . . . . . . . . . . . . . . . 19

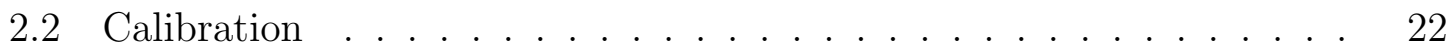

2.2 .1 Effect of Calibration . . . . . . . . . . . . . . 22 
2.2 .2 Joint Angle Errors and Link Errors _. . . . . . . . . . . . . 23

2.2 .3 Repeatability and Accuracy . . . . . . . . . . . . . . . 23

2.2 .4 Nongeometric Error . . . . . . . . . . . . . . . . . . . 24

2.3 Methods of Calibration . . . . . . . . . . . . . . . . . . . . . 25

$2.3 .1 \quad$ Manual Joint Mastering with Indicators _. . . . . . . . . 26

$2.3 .2 \quad$ Manual Joint Mastering with Precise Measurement . . . . . . 26

2.3 .3 Full Pose Measurement . . . . . . . . . . . . . . . . . 27

2.3 .4 Relative Measurements . . . . . . . . . . . . . . . . . . 28

2.3 .5 Measurement using Theodolites . . . . . . . . . . . . . . . 30

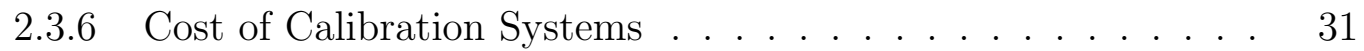

2.3 .7 Fixed Pose Closed Loop Methods . . . . . . . . . . . . . . . 32

2.3 .8 Closed Loop Methods with Fewer Constraints . . . . . . . . . 33

2.3 .9 Closed Loop Methods with Laser Line . . . . . . . . . . . 33

\begin{tabular}{|ll|}
\hline 3 & Experimental Method
\end{tabular} 35

3.1 Robots . . . . . . . . . . . . . . . . . . . . . 35

3.2 Movement of Robot . . . . . . . . . . . . . . . . . . . . . . . . 38

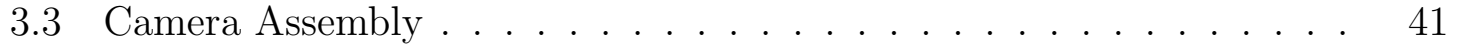

3.3 .1 Geometry of Camera Assembly . . . . . . . . . . . . . 41

3.3 .2 Physical Setup of Camera Assembly . . . . . . . . . . . . 42

3.4 Image Analysis $\ldots \ldots \ldots \ldots \ldots$

$3.4 .1 \quad$ Initial Processing $\ldots \ldots \ldots \ldots \ldots$

3.4 .2 Initial Estimate Using Hough Matrix . . . . . . . . . . . 47

$3.4 .3 \quad$ Finding Regions of Interest (ROI) $\ldots \ldots \ldots \ldots$

3.4.4 Using the Moment Algorithm to Find the Centre and Angle to Sub-Pixel Precision . . . . . . . . . . . . . . . . . . 50

4 Simulation of Calibration Procedure 53

4.1 Purpose of Simulation $\ldots \ldots \ldots \ldots$

4.2 Basic Simulation . . . . . . . . . . . . . . . . . . . . . 53

4.2 .1 Creating a Simulated Data Set _... . . . . . . . 53

4.2 .2 Determining Joint Angle offsets $\ldots \ldots \ldots \ldots \ldots$

4.2 .3 Determining Link Twist offsets $\ldots \ldots \ldots \ldots$

4.2 .4 Results of the Simulated Calibration Procedure . . . . . . 57

4.3 Simulation of Data Acquisition Process _ . . . . . . . . . . 57 
4.3.1 Position of Camera Plane and Obtaining Camera Feedback . . 58

4.3 .2 Adjusting Pose Using Translation in the Tool Frame . . . . . 59

4.3 .3 Adjusting Pose Using Rotation in the Tool Frame . . . . . . . 60

$4.3 .4 \quad$ Full Adjustment at any Pose . . . . . . . . . . . . . . . . . 60

4.3.5 Results of Calibration Using Data Generated with Simulation 61

\begin{tabular}{|lll}
\hline 5 & Experiment Method Refinement & 62
\end{tabular}

5.1 Physical Setup and Experiment $\ldots \ldots \ldots \ldots$

5.1 .1 Adjustment Process . . . . . . . . . . . . . . . . . . . 62

5.1.2 Choosing Adjustment Threshold by Analyzing Repeatability . 63

5.1 .3 Backlash Adjustment . . . . . . . . . . . . . . . 66

5.1 .4 Calibration Time . . . . . . . . . . . . . . . . . . . 66

5.1 .5 Laser Offset Parameters . . . . . . . . . . . . . . . . 67

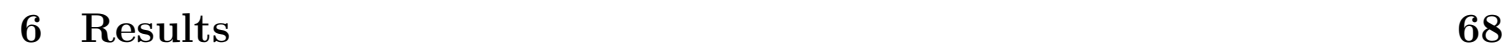

6.1 Simulation Results . . . . . . . . . . . . . . . . . . . . . . 68

6.2 Discussion of Simulation Results . . . . . . . . . . . . . . . . . . 68

6.3 Experimental Results . . . . . . . . . . . . . . . . . . . . . . . . . . . . . 69

6.3 .1 Experiment Using FANUC S-420iF Robot Arm . . . . . . 70

6.3 .2 Experiment Using Motoman MH-180 Robot . . . . . . . . . . 71

6.3 .3 Discussion of Experimental Results . . . . . . . . . . . . . . 73

6.3 .4 Relationship Between Joint 6 Angle Offset and Laser Y Angle Offset . . . . . . . . . . . . . . . . . . . 74

\begin{tabular}{|lll}
\hline 7 & Concluding Remarks & $\mathbf{7 6}$
\end{tabular}

7.1 Summary and Conclusions $\ldots \ldots \ldots \ldots \ldots$

7.2 Recommendations . . . . . . . . . . . . . . . . . . . . 77

\begin{tabular}{ll}
\hline List of References & 79
\end{tabular}

Appendix A Possible Method for Determining Error in Link Length $\begin{array}{lc}\text { and Link Offset } & 84\end{array}$

\begin{tabular}{|ll}
\hline Appendix B MATLAB Code used & 86
\end{tabular}

B.0.1 Data Analysis . . . . . . . . . . . . . . . . . . 86

B.0.2 Main Adjustment Process to Determine Data . . . . . . . . 87 
B.0.3 Rotation Adjustment Code (Y Rotation) . . . . . . . . . . 89

B.0.4 Translation Adjustment Code (Z Translation) . . . . . . . . . 90 


\section{List of Tables}

3.1 Table of robot properties. $\ldots \ldots \ldots \ldots$

4.1 Properties of simulation line. . . . . . . . . . . . . . . . . . 54

4.2 Synthetic error added to DH parameters. . . . . . . . . . . . 54

$4.3 \quad$ Joint angle offset determination. . . . . . . . . . . . . . 57

4.4 Joint twist offset determination. . . . . . . . . . . . . . . . . 57

4.5 Refined joint angle offset determination. $\ldots \ldots \ldots \ldots \ldots$

$4.6 \quad$ Joint angle offset determination for adjustment simulation. . . . . . . 61

6.1 Result of the initial determination of joint angle offsets. . . . . . . . 68

6.2 Result of the modified determination of joint angle offsets. . . . . . 69

6.3 Result of the determination of link twist offsets. . . . . . . . . . . 69

$6.4 \quad$ Joint angle offset result of calibration process using FANUC s-420iF

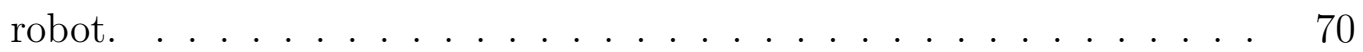

6.5 Laser offset result of calibration process using FANUC s-420iF robot. 70

6.6 Average of results of calibration of FANUC s-420iF robot. . . . . . 71

6.7 Joint angle result of calibration process using Motoman MH-180 robot. 72

6.8 Laser offset result of calibration process using Motoman MH-180 robot. 72

6.9 Average of results of calibration of Motoman MH-180 robot. . . . . . 73

6.10 Difference between Joint 6 angle offset and laser Y angle offset for FANUC s-420iF. . . . . . . . . . . . . . . . . . . . . . . 75

6.11 Difference between joint 6 angle offset and laser $\mathrm{Y}$ angle offset for Motoman MH-180. . . . . . . . . . . . . . . . . . . . 75 


\section{List of Figures}

$2.1 \quad$ A Delta Flex Picker robot. . . . . . . . . . . . . . . . . . . 8

2.2 The 6 degree-of-freedom Gough tire-test platform from 1956. . . . . . 9

2.3 The Kuka KR-15/2. $\ldots \ldots \ldots \ldots$

2.4 A six-axis wrist-partitioned robot. . . . . . . . . . . . . . 11

$2.5 \quad$ A hybrid robot comprised of four algebraic screw pairs. . . . . . . . 12

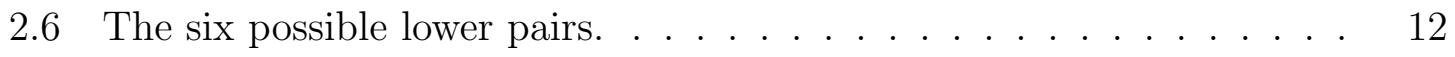

2.7 DH parameters and reference frame attachment on a link. . . . . . 14

2.8 DH parameters shown on Kuka KR-15/2. . . . . . . . . . . . 15

3.1 Fanuc S-420iF robot arm. . . . . . . . . . . . . . . . 36

3.2 Motoman MH-180 robot arm. . . . . . . . . . . . . . . 37

3.3 Flowchart describing calibration process. . . . . . . . . . . . . . 39

$3.4 \quad$ Flowchart describing single adjustment. . . . . . . . . . . . . . 40

3.5 Use of beam splitter and two cameras. . . . . . . . . . . . . . 42

3.6 Constructed camera assembly. . . . . . . . . . . . . . . . . . . . . 43

3.7 Photo of camera assembly. . . . . . . . . . . . . . . . . . . . 44

3.8 Input image. $\ldots \ldots \ldots \ldots \ldots \ldots$

3.9 Input image converted to black and white. . . . . . . . . 46

3.10 Result of hough transform on cross image. . . . . . . . . . . . 48

3.11 Regions of interest on cross image. $\ldots \ldots \ldots \ldots . \ldots . \ldots 49$

3.12 Final cross centre determination. . . . . . . . . . . . . . . . . 52

5.1 Point spread on Camera 1 of repeatability test. . . . . . . . . . . 64

5.2 Point spread on Camera 2 of repeatability test. . . . . . . . . . 65 


\section{Chapter 1}

\section{Introduction}

\subsection{Motivation}

Calibration drastically increases the accuracy of a robot, allowing it to autonomously perform tasks that would otherwise be impossible. Calibration is a process in which measurements taken by a specific device are compared to known measurements, in order to determine the accuracy of the measurements taken by the device, and increase this accuracy by compensating for these errors. Calibration can reduce the error in a robot's movement without physically modifying the robot. Very high accuracy is becoming more important for robotic manipulators. Many high precision tasks are now being accomplished with robotic arms [1]. Robotic manipulators are frequently used for surgical procedures, in which minor errors can severely affect patients [2]. It would be impossible to perform at this level of precision without calibration. Precision manufacturing (such as small electronics) can also be accomplished with a robotic manipulator. In order to operate with the required precision with a robot, calibration must be performed to identify and compensate for errors in the kinematic model.

When manufacturing robots were first intruduced in factories in the 1960s, they were used for simple pick-and-place tasks, with poses that were taught manually. An operator would specify the exact motion the robot would perform, and then that motion would be performed repeatedly. This type of task is easily accomplished without calibration because it relies on the robot's repeatability. Repeatability is a measure of a robot's ability to return to a known position, whereas accuracy is a measure of a robot's ability to move to a position that was not previously taught [3]. The difference between these two values is caused by errors in the robot's kinematic model. For a taught position, the values of the robot's joint angles, as measured 
by the controller, are stored and the joints simply return to these exact positions. When moving to a new position, the joint angle values must be calculated. This calculation depends on the kinematic model of the robot: the measured values of its geometry. The error in these values will cause inaccuracy when moving to this new position. For this reason, the value of repeatability will always be better than the value of accuracy. Typically, repeatability will be an order of magnitude better than accuracy. However, accuracy varies highly between robots. Repeatability tends to scale with the overall size of a robot [4], but remains quite small. For instance, a Kuka KR 15/2 robot arm has a forward reach of $1570 \mathrm{~mm}$, and a repeatability of $\pm 0.1 \mathrm{~mm}$ [5]. The smaller IRB 120 from ABB has a forward reach of $580 \mathrm{~mm}$, and a repeatability of $\pm 0.01 \mathrm{~mm}[6]$. The theoretical best possible accuracy that can be achieved is the repeatability, because if the robot's kinematic model is exactly correct, it will be able to move to new positions as accurately as previously taught positions.

As robotics became more ubiquitous in factories, more complex and exact motions were required. Manipulator calibration is essential for tasks that require a robot to move to a pose that has not been manually taught, such as any task that requires sensing a location and moving to that location. Calibration is a process by which the accuracy of the robot can be substantially increased by modifying only the software controlling the robot's movement. The goal of a calibration routine is to identify the small differences between the robot's geometry as it is recorded in the controller software and the actual physical dimensions of the robot.

There is typically a balance between expense and accuracy in calibration routines. The necessary measurement tools can never provide a perfectly accurate picture of the real robot geometry. Some error will always exist. The precision of measurements will affect the success of the calibration routine, and the accuracy of its results. A very high accuracy measurement, of any length, angle, or other geometric feature of a robot, can be very expensive due to the cost of highly accurate sensing equipment. Calibration routines typically employ complex mathematics to reduce the necessary amount of measurements without substantially decreasing the accuracy of the calibration routine.

The calibration method presented in this thesis uses a novel measurement system and error identification method using a small number of inexpensive sensors. The robots to be calibrated were located at Enclosures Direct Inc., a local business which manufactures electrical enclosures. The manufacturing process involves punching a 
shape from a sheet of metal, bending the metal into the desired shape, welding the seams, and applying a foam gasket at the edges of the door of the enclosure. The welding and the foam gasket application are both performed by robotic arms. These electrical enclosures are all manufactured to custom specifications from clients. As such, a different set of positions is required for the manufacture of each individual enclosure, so using manually taught positions to accomplish these tasks is impossible. The welding task relies heavily on an operator. The operator places the enclosure with the seam in a specific position, and the robot, using a laser vision system, determines the location and length of this seam and welds it. The operator must then reposition the entire enclosure to weld another seam. This limits the speed at which the welding can be performed, as the enclosure must be moved back and forth manually several times during welding. The size of enclosures is also limited, as each enclosure must not be too heavy to be lifted by a single operator. With calibration, a better welding process could be developed that would allow the robot to move to several different positions and weld each seam without the operator having to reposition the enclosure.

Additional challenges for calibration are presented by the environment in which the calibration must be performed. An active factory floor presents many difficulties to sensing equipment. The most challenging of these is the heavy machine vibrations. The sheet metal punching was performed by a punch press. These vibrations cause problems for any sort of sensing equipment. Additionally, a large amount of dirt and dust causes problem for cameras, which are used in the calibration process presented in this thesis. Measures to compensate for these problems are discussed in Chapter 5 .

The calibration method presented herein is designed to identify the errors in only part of the kinematic model: the joint angle error and the link twist offset. These account for the majority of the error in a robot's position and orientation (pose) [7. However, additional error can be caused by many factors, such as differences in temperature [8].

\subsection{Objectives}

There are three main objectives in this thesis:

1. Develop a simulation to verify the calibration methodology, which is a modified form of the virtual linear constraint [9] (discussed in Subsection 2.3.9) with an additional constraint on rotation about this line (discussed in Chapter 3). 
2. Develop a physical setup and measurement system that will allow for the virtual constraints to be applied. In order to constrain rotational motion about the virtual line, this rotational motion must be measured. This is described in Chapter 3 .

3. Use this setup to calibrate a physical robot.

\subsection{Statement of Originality}

To the best of the knowledge of the author the following contributions presented in this thesis stem from original ideas and results and are presented herein for the first time.

1. Constraint of rotation about the laser line during calibration using a cross-shape laser line optics in place of a typical straight laser line.

2. Design of a two-camera assembly to read changes in a robot's pose.

3. Design of an image processing algorithm allowing for precise measurement of small changes in position and orientation of a cross shape on an image.

4. Design of a motion pattern to establish robot poses that can be attained and such that the virtual constraints are satisfied.

\subsection{Thesis Overview}

- In Chapter 2, the theory behind robotic arm movement and kinematic calibration are discussed. Methods of calibration are discussed, from simple to complex. The methods upon which part of this thesis is based are identified and described.

- In Chapter 3, the overall experimental method that will be used in a simulation is described. This includes the way in which data will be gathered from the robots, as well as the ways in which camera feedback will be analyzed.

- In Chapter 4, two calibration simulations are described. In the first simplified simulation, sample data is generated automatically to match the desired movement pattern of the robot, and the calibration is performed with this sample 
data. In the second, more complex simulation, the full process of data gathering is simulated, including each robot movement, and the camera feedback.

- In Chapter 5, modifications to the experimental method given in Chapter 3 are discussed. These are modifications that are not included in the computer simulation. The physical experiment required several modifications to the overall process in order to function.

- In Chapter 6, results are shown. This includes results of the simple and complex simulations, as well as experimental results. A discussion of these results is provided.

- Chapter 7 presents a summary and conclusions, as well as recommendations. 


\section{Chapter 2}

\section{Theory and Literature Review}

This chapter provides the necessary background. A review of existing literature on calibration methods is also included. Original contributions are mentioned in context of existing literature, and literature contribution to original work is discussed.

\subsection{Manipulator Kinematics}

In order to calibrate a serial robot, the movement of the robot must be modeled. In this section, the basic kinematics used to represent robot movement will be discussed for the specific robot type on which the calibration is performed. Later in this chapter, the descriptions presented here will be developed in detail.

\subsubsection{Overview of Manipulator Kinematics}

Kinematics is defined as the science of motion without reference to the forces which cause that motion. In order for a robot's motion to be analyzed, it must first be fully described. This is done by representing the position and orientation (or "pose") of each part of the robot. For a serial robotic manipulator like the ones used in this thesis, the position and orientation of each link must be described. These poses are described using a coordinate system, which must include a reference frame. Each pose is expressed relative to this reference frame. In the process of describing an entire robot, many different reference frames are used. As such, it is important to describe the way to take a pose in one reference frame and determine what that pose would be in a different reference frame. This is referred to as "transforming" the coordinates.

The parts of the robot which are being described are the links. Joints connect 
these links, which allow relative motion of adjacent links in specific ways. This motion may be a rotation, in the case of a revolute joint, or a translation, in the case of a prismatic joint. These, and other joints types are described in Subsection 2.1.3.

Forward kinematics describes the process by which the pose of the end effector (the end of the final link) is found relative to the base of the robot, using the robot's geometry and the angle, or offset of each joint. This is done by assigning a reference frame to both the end effector and the base of the robot (the "tool frame" and the "base frame", or "world frame," as the base remains stationary). In order to do this, an individual reference frame is attached to each link of the robot, and a transformation is performed from one reference frame to the next for each link. This process is described in Subsection 2.1.7.

Inverse kinematics is the opposite process. Given a desired pose, the necessary joint values are calculated. For a serial robot such as the ones used in this thesis, a general solution to the inverse kinematics can be mathematically very complex. Depending on the desired pose, there are likely multiple solutions to the inverse kinematics problem. However, depending on the geometry of the desired robot, the solution can be simplified. This is described in Subsection 2.1.8.

\subsubsection{Types of Robotic Manipulators}

There are a wide variety of robotic manipulators. Several will be discussed in this subsection. While some resemble the structure of a human arm and hand, others may have substantially different structure. Parallel manipulators are supported by multiple kinematic chains [10], for example, the Delta Flex Picker robot shown in Figure 2.1. A well-known example of a parallel manipulator is the Gough-Stewart Platform [11,12], a platform supported by six parallel active prismatic joints, shown in Figure 2.2. These may offer higher accuracy [13], but have a smaller workspace and a more complex kinematic model. A serial manipulator has a more conventional form, usually with a shape resembling a human arm, such as the Kuka KR 15/2 shown in Figure 2.3. These offer a larger workspace but overall lower accuracy, and lower stiffness [13.

A common type of serial manipulator has six revolute joints, though there are many configurations with different numbers and types of joints. The two robots used in this thesis, a Fanuc S-420iF [14], and Motoman MH-180 [15] are both wristpartitioned serial manipulators with six revolute joints. A wrist-partitioned robot 
has its last three joints orient the end-effector and have revolute joint axes that intersect at the "wrist point", while the first three joints position the wrist point [3]. Figure 2.4 shows a six-axis wrist-partitioned serial robot. The Kuka KR-15/2 in Figure 2.3 is also a wrist-partitioned robot. Some manipulators use a hybrid serialparallel configuration [16:17] in order to keep the advantages of a parallel manipulator but extend the available workspace. Figure 2.5 shows a hybrid robot with "algebraic screw pair" joints - single degree-of-freedom joints which rotate and translate in a coupled way $[18$.

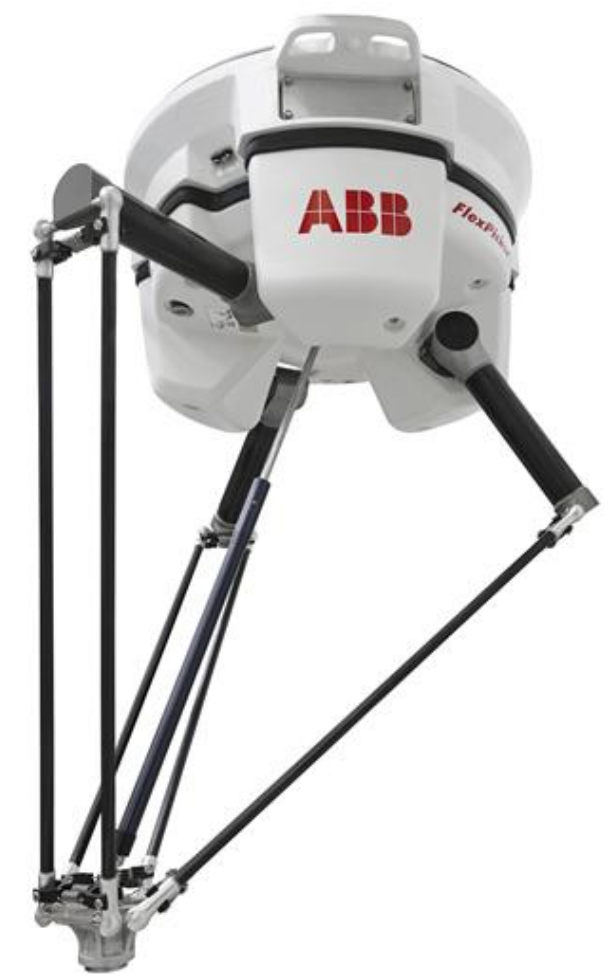

Figure 2.1: A Delta Flex Picker robot. 


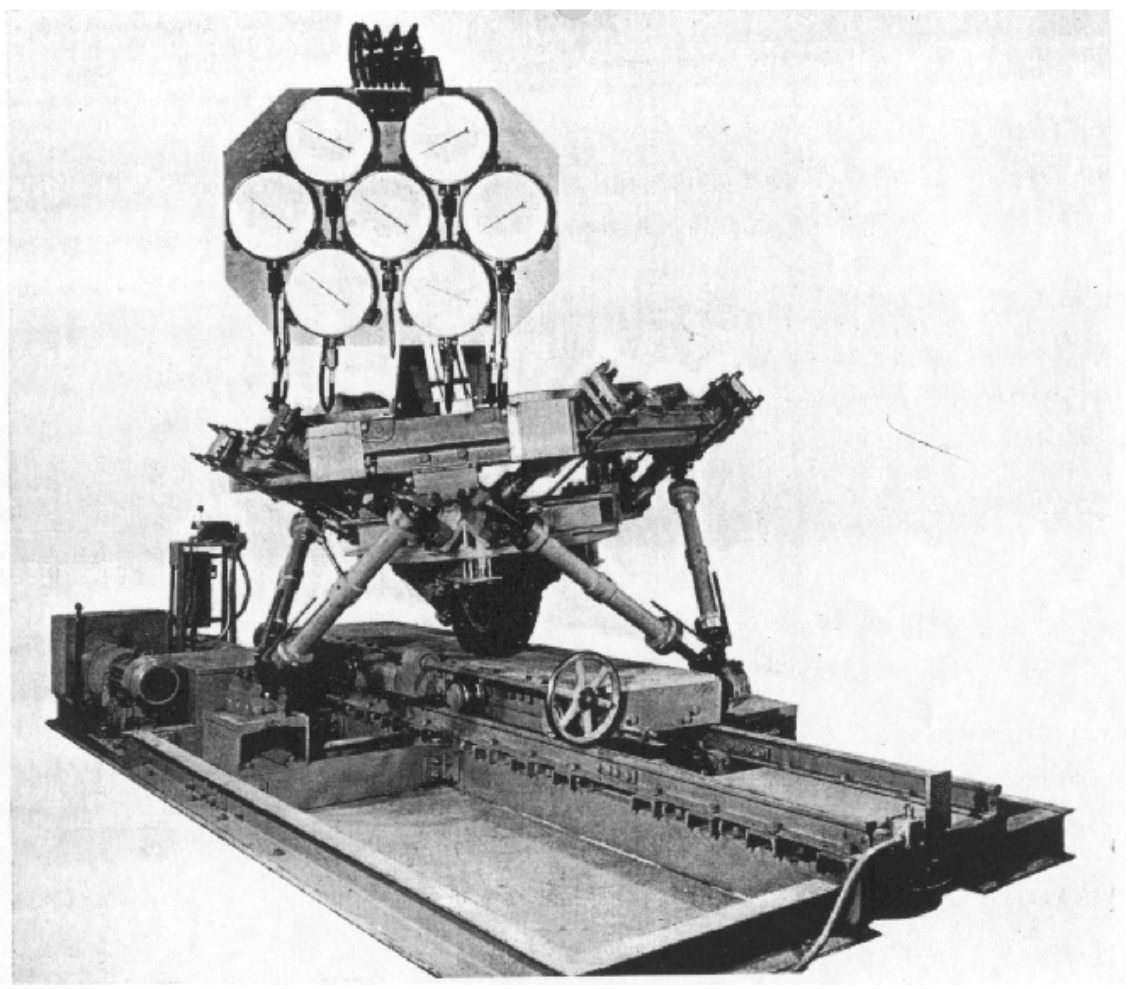

Figure 2.2: The 6 degree-of-freedom Gough tire-test platform from 1956. 


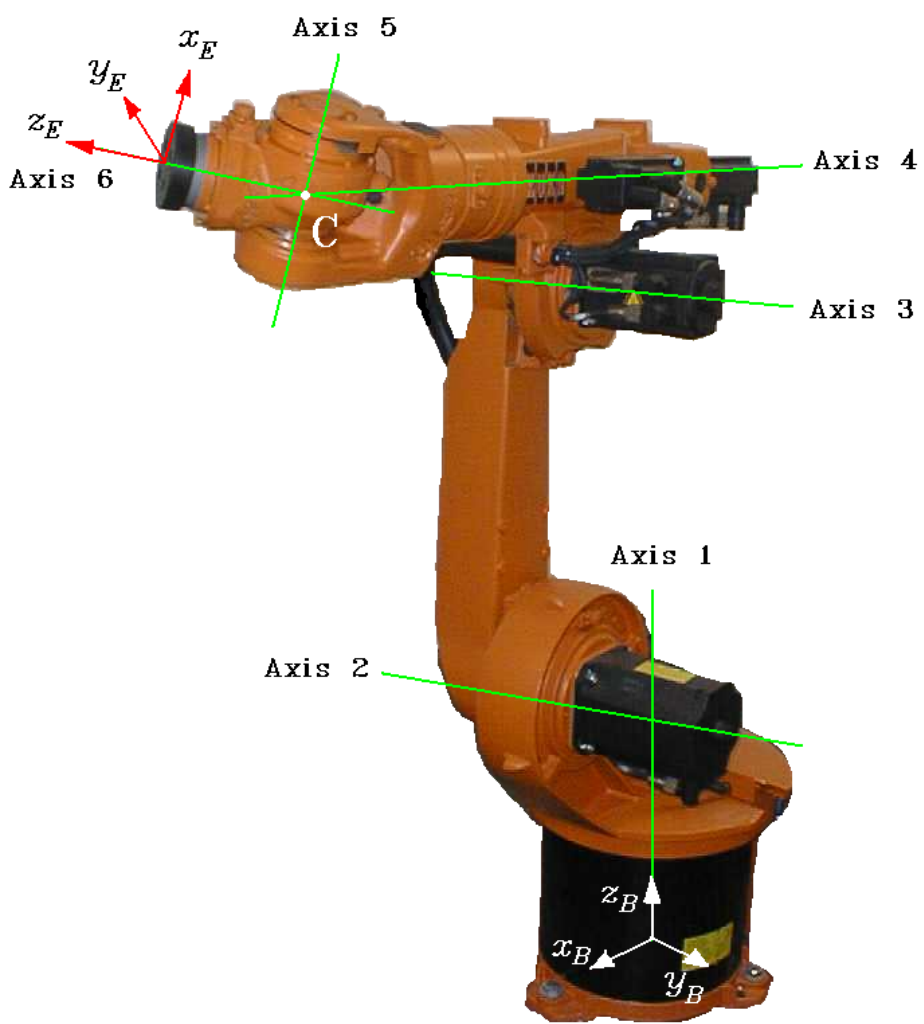

Figure 2.3: The Kuka KR-15/2. 


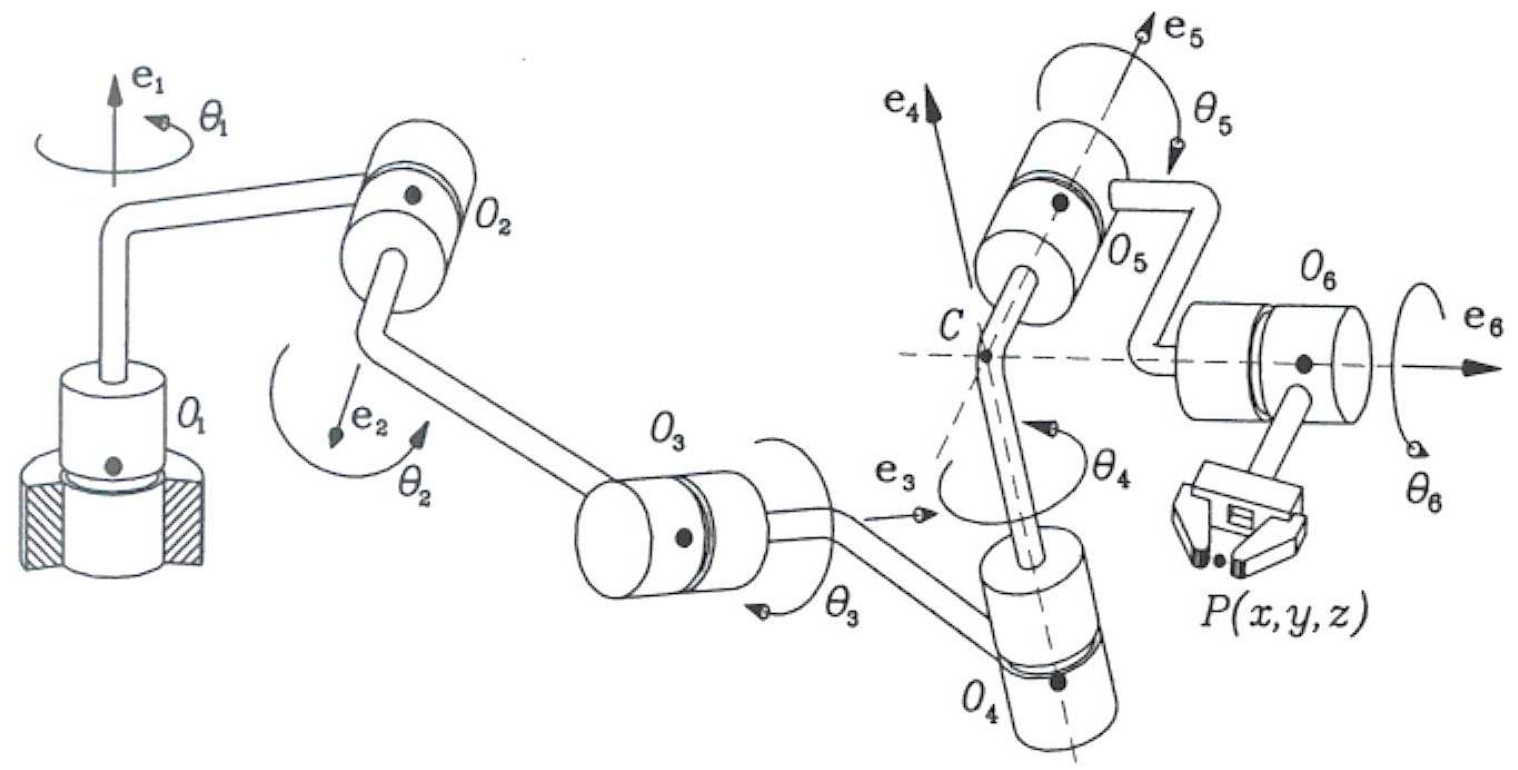

Figure 2.4: A six-axis wrist-partitioned robot.

\subsubsection{Joints}

A joint is a mechanical way of constraining the relative motion between one link of a robot and the next. A lower pair is a term used to describe a connection between two bodies which have surface contact relative to each other [3]. In order to accurately represent the movement of a manipulator, the structure of the manipulator needs to be fully described. The structure of a serial manipulator is a sequence of links connected by joints. There are a variety of different types of joints, with varying degrees of freedom. The two most common joint types are revolute and prismatic joints, each with a single degree of freedom. A revolute joint allows rotation about a single axis. A prismatic joint allows translation along a specific axis. Both of these are illustrated in Figure 2.6 (modified from [19]).

There are six types of lower pairs in total, with varying degrees of freedom, though the remaining four will not be discussed here. The robots used in this thesis have only revolute joints. 


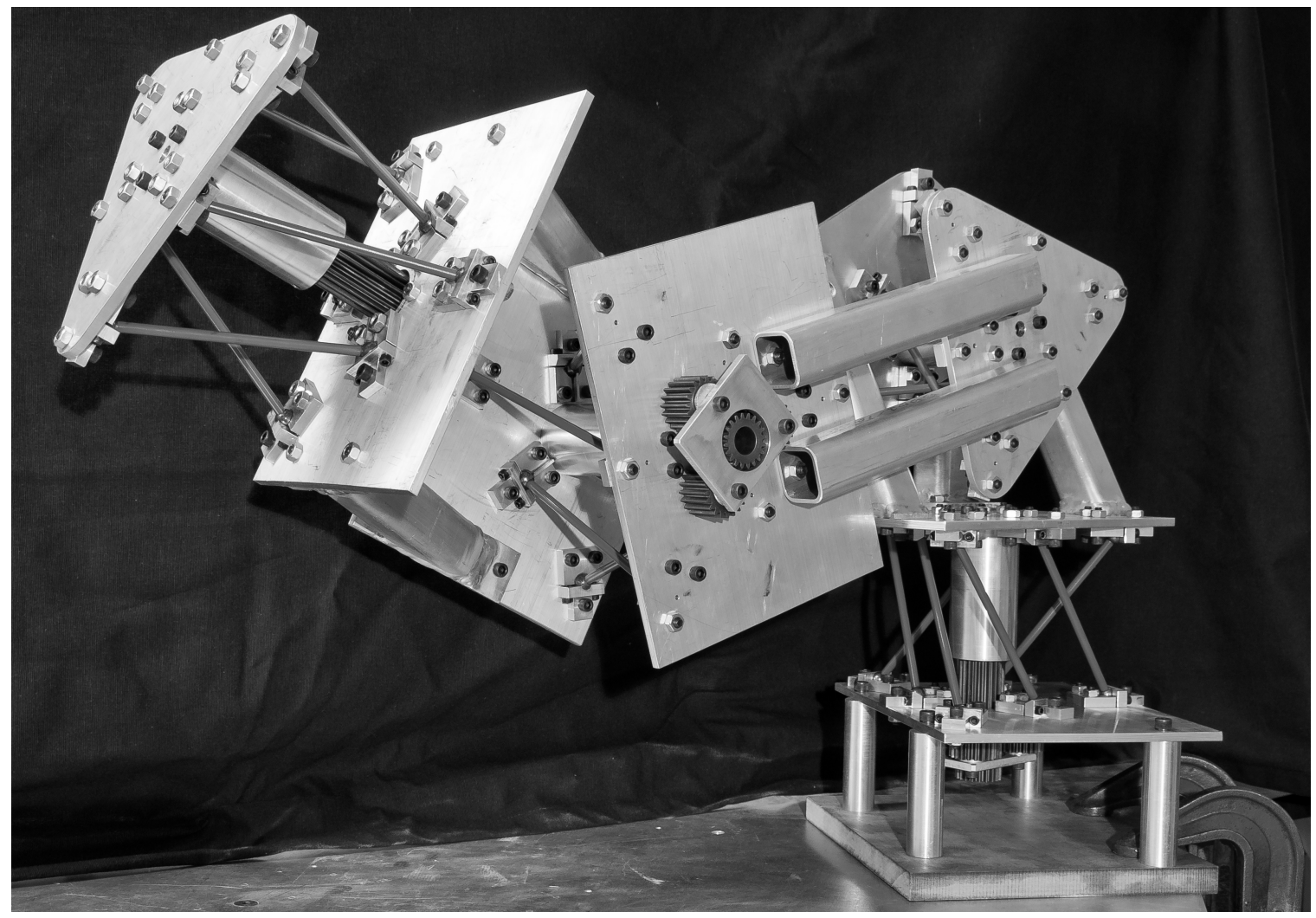

Figure 2.5: A hybrid robot comprised of four algebraic screw pairs.

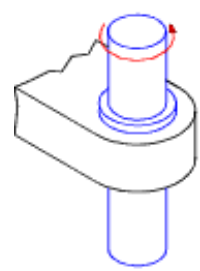

Revolute

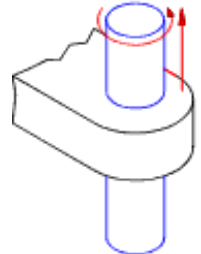

Cylindrical

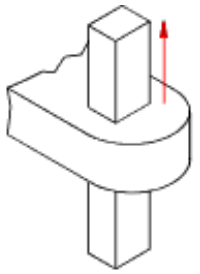

Prismatic

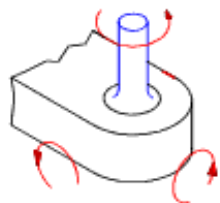

Spherical

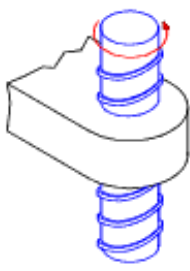

Screw

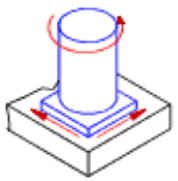

Planar

Figure 2.6: The six possible lower pairs. 


\subsubsection{Attachment of Reference Frames to Links}

The most commonly used representation of robot geometry, as created by Denavit \& Hartenberg [20] involves attaching a reference frame to each link of the robot and using certain parameters to describe the relative position of these reference frames. For each link on the robot, there is a reference frame, attached in a specific position to describe the movement of the joint between the link and the surrounding links. The modified method for attaching these frames for a given link (link $i$ ) is given below. This procedure is as described by Craig [3]. The difference between the original and modified methods is described in Subsection 2.1.6.

- The reference frame is attached rigidly to link $i$ (i.e. the reference frame will move with the link).

- $\hat{Z}_{i}$ is placed such that it corresponds with the axis of rotation of the joint between link $i$ and link $i-1$.

- The origin of the frame is placed at the intersection of $\hat{Z}_{i}$ and $\hat{X}_{i-1}$.

- $\hat{X}_{i}$ is placed along the common normal between $\hat{Z}_{i}$ and $\hat{Z}_{i+1}$ (or, normal to the plane created by $\hat{Z}_{i}$ and $\hat{Z}_{i+1}$, should they intersect).

- $\hat{Y}_{i}$ is placed following the right-hand rule, once $\hat{Z}_{i}$ and $\hat{X}_{i}$ have been placed.

The base frame is then assigned so that it corresponds exactly to the frame of link 1 , when the first joint angle is zero. The tool frame is assigned such that the tool $X$ axis $\left(\hat{X}_{n}\right)$ aligns with $\hat{X}_{n-1}$ when the last joint angle is zero. The frame origin is assigned such that $d_{n}$ is zero. A depiction of the frame attachment for an arbitrary link is shown in Figure 2.7.

\subsubsection{Denavit-Hartenberg Parameters}

Once the reference frames for each joint have been assigned, the structure and position of the robot can be described entirely through a set of parameters. These are known as Denavit-Hartenberg parameters (or simply DH parameters), of which there are 24 total for a six-axis serial arm [20]. For each link, there are four parameters. The definition of these parameters is given below: 


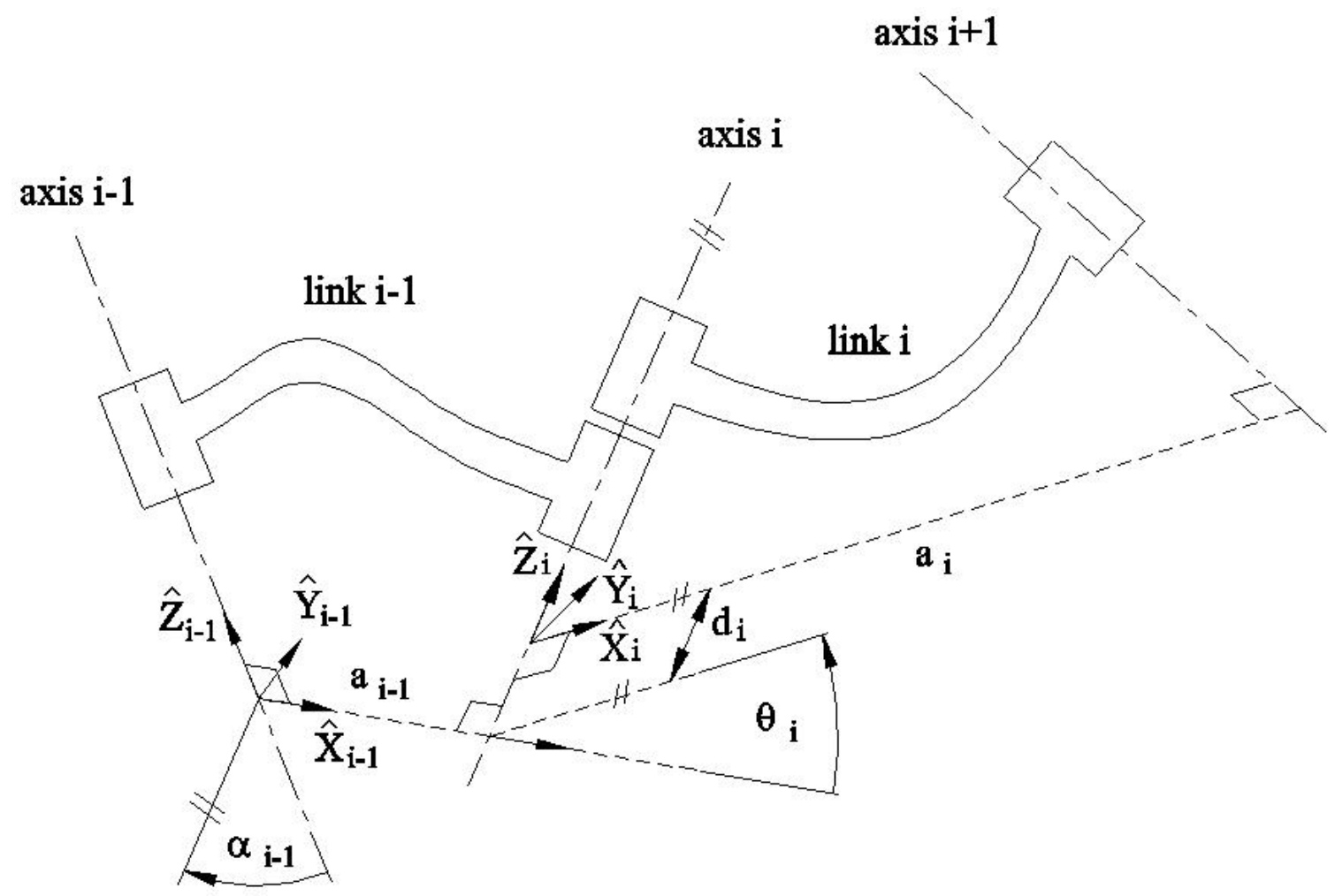

Figure 2.7: DH parameters and reference frame attachment on a link. 
- Link Length $\left(a_{i}\right)$ : the distance from $\hat{Z}_{i}$ to $\hat{Z}_{i+1}$, along $\hat{X}_{i}$.

- Link Offset $\left(d_{i}\right)$ : the distance from $\hat{X}_{i-1}$ to $\hat{X}_{i}$, along $\hat{Z}_{i}$.

- Link Twist $\left(\alpha_{i}\right)$ : the angle from $\hat{Z}_{i}$ to $\hat{Z}_{i+1}$, about $\hat{X}_{i}$.

- Joint Angle $\left(\theta_{i}\right)$ : the angle from $\hat{X}_{i-1}$ to $\hat{X}_{i}$, about $\hat{Z}_{i}$.

These parameters are shown applied to a Kuka KR-15/2 robot in Figure 2.8. The parameters are used to fully describe the position and orientation of each reference frame relative to the previous reference frame. See Figure 2.7 for example. During the operation of the robot, movement is made by rotating the joints, which changes the joint angles $(\theta)$. The other 3 parameters $(a, d$, and $\alpha)$ are inherent in the construction of the robot, and ideally would remain constant for each link. In practice, there can be some variation in these parameters, which will be discussed further in Subsection 2.2.4.

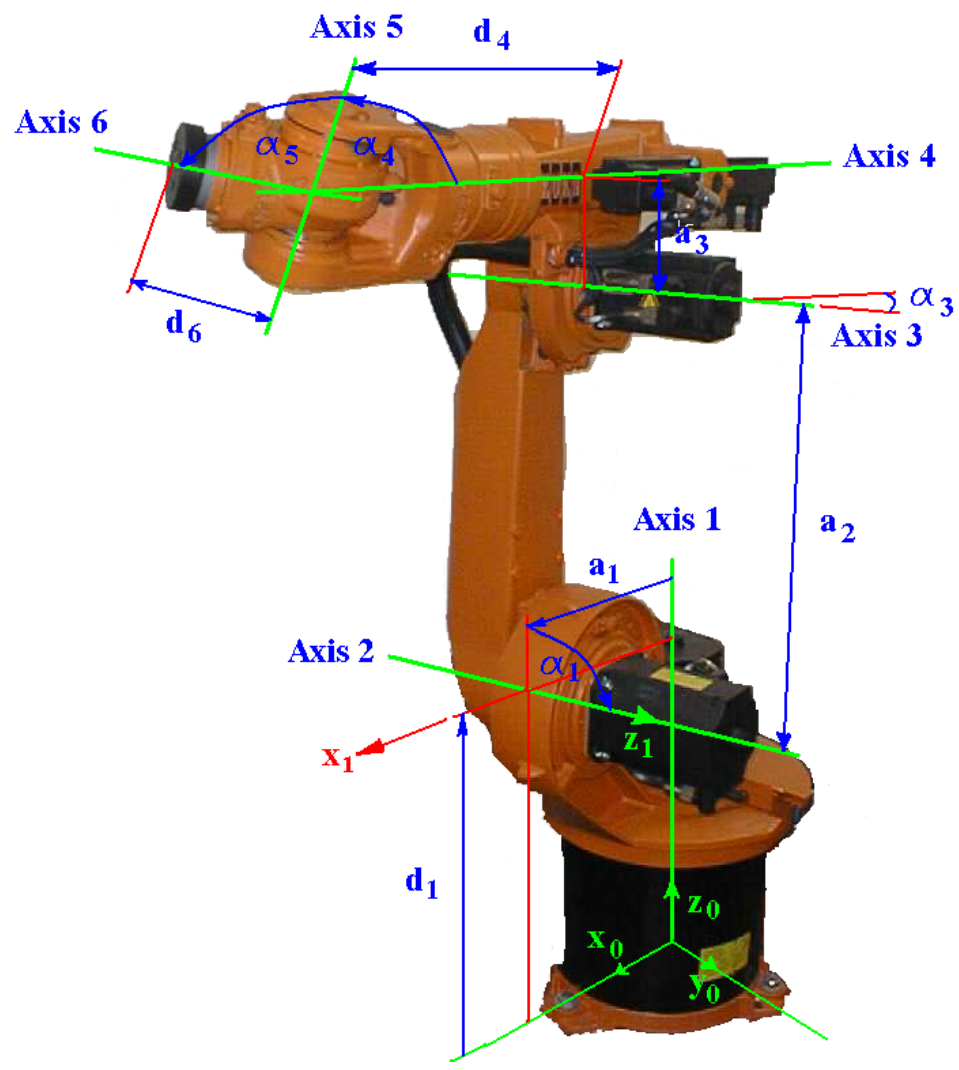

Figure 2.8: DH parameters shown on Kuka KR-15/2. 
The parameters are used to fully describe the position and orientation of each reference frame relative to the previous reference frame. See Figure 2.7 for example. During the operation of the robot, movement is made by rotating the joints. This changes the joint angles $(\theta)$. The other 3 parameters $(a, d$, and $\alpha$ ) are inherent in the construction of the robot, and ideally would remain constant for each link. In practice, there can be some variation in these parameters, which will be discussed further in Subsection 2.2.4.

\subsubsection{Variation in DH Parametrizations}

There are several variants of the DH parameter notation in use. The original notation given in [20] was developed primarily for use in single closed loop mechanisms (a closed loop mechanism is one such that the last link and the first link are connected to each other, forming a loop). Modified versions are better designed to work for an open loop mechanism such as a robot arm. These are referred to as modified DH parameters. The notations presented here use modified DH parameters. A comparison of the original DH notation and two forms of modified DH parameters can be found in 21], including distal and proximal variants. The proximal variant is the form used here. "Distal" and "proximal" here describe the placement of the reference frames on links. The distal variant places frame $i$ on the distal end of link $i$ (where link $i$ meets link $i+1$ ) and the proximal variant places frame $i$ at the proximal end of link $i$ (where link $i$ meets link $i-1)$. The primary advantage of the proximal variant is transparency. The parameters for link $i$ are measured along the axes of frame $i$. In the original $\mathrm{DH}$ notation and the distal variant, some parameters are measured along axes of the previous joint. A disadvantage of the proximal variant is the use of multiple indices in calculating the position of the next reference frame. The values used in determining the transformation from frame $i-1$ to frame $i$ are: $\theta_{i}, d_{i}, \alpha_{i-1}$, and $a_{i-1}$. In the original and distal variant, the required values would be $\theta_{i}, d_{i}, \alpha_{i}$, and $a_{i}$. These transformations are discussed in detail in Subsection 2.1.7.

\subsubsection{Forward Kinematics}

The method for determining a reference frame transformation, from the base frame to the end effector reference frame, is referred to as forward kinematics. Forward kinematics is the transformation from joint space to cartesian space, which implies 
transforming a set of joint angles to the position and orientation of the end effector in the base coordinate system. The process of forward kinematics takes the known DH parameters of a specific robot at a specific position, and uses them to calculate the position and orientation of the robot's end effector, usually in world frame coordinates. A set of transformation matrices is calculated, each one representing the transformation of a point from one reference frame to the next. The transformation from frame $i-1$ to frame $i$ is represented by ${ }_{i}^{i-1} A .{ }_{i}^{i-1} A$ can be calculated from $\mathrm{DH}$ parameters using Equation 2.1. (Note that here $\mathrm{c}$ and $\mathrm{s}$ are used as abbreviations for $\cos$ and $\sin )$ :

$$
{ }_{i}^{i-1} A=\left[\begin{array}{cccc}
c \theta_{i} & -s \theta_{i} & 0 & a_{i-1} \\
s \theta_{i} c \alpha_{i-1} & c \theta_{i} c \alpha_{i-1} & -s \alpha_{i-1} & -s \alpha_{i-1} d_{i} \\
s \theta_{i} s \alpha_{i-1} & c \theta_{i} s \alpha_{i-1} & c \alpha_{i-1} & c \alpha_{i-1} d_{i} \\
0 & 0 & 0 & 1
\end{array}\right] .
$$

For a robot with six joints, this will yield six matrices which, when multiplied together, form a transformation matrix from the base frame to the reference frame attached to the robot's end effector, see Equation 2.2. There may, in some cases, be an additional transformation matrix to account for a specific tool attached to the robot's arm $\left(A_{\text {tool }}\right)$. $A_{\text {tool }}$ is specific to the tool attached and remains constant throughout the process of moving the robot.

$$
\left[\begin{array}{cccc}
T_{11} & T_{12} & T_{13} & T_{14} \\
T_{21} & T_{22} & T_{23} & T_{24} \\
T_{31} & T_{32} & T_{33} & T_{34} \\
0 & 0 & 0 & 1
\end{array}\right]=T={ }_{1}^{0} A_{2}^{1} A_{3}^{2} A_{4}^{3} A_{5}^{4} A_{6}^{5} A A_{\text {tool }} .
$$

The position of the end effector reference point, typically the origin of the end 
effector frame, in base frame coordinates, is given by Equation 2.3 .

$$
\left[\begin{array}{l}
X \\
Y \\
Z
\end{array}\right]=\left[\begin{array}{l}
T_{14} \\
T_{24} \\
T_{34}
\end{array}\right]
$$

The orientation of the end effector is also embedded in T. The orientation is usually expressed using 3 angles, $W, P$, and $R$, relative to three linearly independent coordinate axes, where the top left $3 \times 3$ submatrix of $T$ is the product of 3 elemental rotation matrices, as expressed in Equation 2.4, for example:

$$
\left[\begin{array}{lll}
T_{11} & T_{12} & T_{13} \\
T_{21} & T_{22} & T_{23} \\
T_{31} & T_{32} & T_{33}
\end{array}\right]=R z(R) R y(P) R z(W) .
$$

Where $R x, R y$, and $R z$ are 3-dimensional rotation matrices about the $x, y$, and $z$ axes, respectively. Note that these equations use atan 2 , a function available in many programming languages, including MATLAB (which was used here). This function calculates the four-quadrant inverse tangent, which accounts for the sign of the inputs to determine the result.

To find the values of $W, P$, and $R$ from T, Equations 2.5, 2.6, and 2.7 can be used:

$$
\begin{aligned}
& P=\operatorname{atan} 2\left(-T_{31}, \sqrt{T_{11}^{2}+T_{21}^{2}}\right), \\
& W=\operatorname{atan} 2\left(\frac{T_{32}}{\cos (P)}, \frac{T_{33}}{\cos (P)}\right), \\
& R=\operatorname{atan} 2\left(\frac{T_{21}}{\cos (P)}, \frac{T_{11}}{\cos (P)}\right) .
\end{aligned}
$$

The values determined for $X, Y, Z, W, P$, and $R$ are the final result of the forward kinematics. Starting with 18 parameters describing the robot's structure $(a$, $d$, and $\alpha$ values), the tool transformation matrix $\left(A_{\text {tool }}\right)$, and the six joint angles $(\theta)$, the final position and orientation of the robot tool refence frame is determined $(X$, $Y, Z, W, P, R)$. 


\subsubsection{Inverse Kinematics}

Inverse kinematics is the process through which we determine the set of joint angles which will place the end effector at a specific position and orientation. This is the inverse of the forward kinematics. The known values are the 18 parameters describing the robot's structure ( $a, d$, and $\alpha$ values), the tool transformation matrix $\left(A_{\text {tool }}\right)$, and the desired end effector position and orientation $(X, Y, Z, W, P, R)$. The result of this computation will be the six joint angles $(\theta)$ required to place the end effector at this position and orientation.

The inverse kinematics for serial manipulators is substantially more mathematically complex than the forward kinematics. For any given end effector position, there will not necessarily be a single correct set of joint angles. For a robot with six revolute joints, there may be up to sixteen different solutions for a given pose [3]. There may also be no solution, for a pose that is not reachable by the robot.

The general solution to this problem for a robot with six revolute joints is quite complex. Various methods exist for solving the general inverse kinematics, such as 22, 23. However, certain special cases exist that allow for simplified solutions. The robots used here have a specific structure in which the last 3 joint axes intersect in a single point, referred to as the "wrist" point. For this special case, a simplified solution was created by Pieper 24]. The equations presented here are a modified version of those presented in [3].

The $T$ matrix for the whole system must be calculated from the position and orientation $(X, Y, Z, W, P, R)$, using Equations 2.3 and 2.4 in Subsection 2.1.7. In this case, the $T$ matrix is modified to exclude $A_{\text {tool }}$, as shown in Equation 2.8:

$$
T=T_{\text {actual }} A_{\text {tool }}^{-1} .
$$

From this new $T$ matrix, the end effector's XYZ position $\left(p_{e e}\right)$ can be computed using Equation 2.3. Following this, the wrist point $\left(p_{\text {wrist }}\right)$ is calculated using Equation 2.9:

$$
p_{\text {wrist }}=p_{e e}-d_{6} T_{z} .
$$

where $d_{6}$ is the offset of the last link, and $T_{z}$ is the $\mathrm{z}$ column of the rotation matrix in $T$ (the first 3 entries of the third column of $T$ ). 
The first angle, $\theta_{1}$, is found simply using Equation 2.10:

$$
\frac{p_{\text {wrist }, X}}{p_{\text {wrist }, Y}}=\frac{\cos \left(\theta_{1}\right)}{\sin \left(\theta_{1}\right)} .
$$

Next, the calculation for $\theta_{3}$ is performed. It is more complex and requires several equations. First, the squared magnitude of the wrist position is calculated in Equation 2.11. Following this, the possible values of $\theta_{3}$ are the solutions to Equation 2.12, which depends on $k$ values and $f$ values given in Equations 2.13 and 2.14

$$
\begin{gathered}
r=p_{\text {wrist }, X}^{2}+p_{\text {wrist }, Y}^{2}+p_{\text {wrist }, Z}^{2}, \\
\frac{\left(r-k_{3}\right)^{2}}{4 a_{1}^{2}}+\frac{\left(p_{\text {wrist }, Z}-k_{4}\right)^{2}}{\sin ^{2} \alpha_{1}}=k_{1}^{2}+k_{2}^{2}, \\
k_{1}=f_{1}+a_{2}, \\
k_{2}=\sin \left(\alpha_{2}\right) f_{3}-\cos \left(\alpha_{2}\right) f_{2}, \\
k_{3}=f_{1}^{2}+f_{2}^{2}+f_{3}^{2}+a_{1}^{2}+a_{2}^{2}+2 f_{1} a_{2}, \\
k_{4}=f_{2} \cos \left(\alpha_{1}\right) \sin \left(\alpha_{2}\right)+f_{3} \cos \left(\alpha_{1}\right) \cos \left(\alpha_{2}\right), \\
f_{1}=\sin \left(\theta_{3}\right) \sin \left(\alpha_{3}\right) d_{4}+a_{3} \cos \left(\theta_{3}\right), \\
f_{2}=a_{3} \sin \left(\theta_{3}\right)-\cos \left(\theta_{3}\right) \sin \left(\alpha_{3}\right) d_{4}, \\
f_{3}=\cos \left(\alpha_{3}\right) d_{4}+d_{3} .
\end{gathered}
$$

Combining these $f$ and $k$ values in Equation 2.12 produces a large equation which relies on $\theta_{3}$, but not on any of the other joint angles.

At this point, the calculation for $\theta_{2}$ is performed. Only Equation 2.15 is required here. This equation is solved using all possible values of $\theta_{1}$ and $\theta_{3}$, to get all possible 
sets of the first 3 joint angles.

$$
\begin{aligned}
P_{\text {wrist }, y}= & \left(\left(\sin \left(\theta_{1}\right) \cos \left(\theta_{2}\right)+\cos \left(\theta_{1}\right) \cos \left(\alpha_{1}\right) \sin \left(\theta_{2}\right)\right) \cos \left(\theta_{3}\right)+\left(-\sin \left(\theta_{1}\right) \sin \left(\theta_{2}\right) \cos \left(\alpha_{2}\right)\right.\right. \\
& \left.\left.+\cos \left(\theta_{1}\right) \cos \left(\alpha_{1}\right) \cos \left(\theta_{2}\right) \cos \left(\alpha_{2}\right)-\cos \left(\theta_{1}\right) \sin \left(\alpha_{1}\right) \sin \left(\alpha_{2}\right)\right) \sin \left(\theta_{3}\right)\right) a_{4} \\
& +\left(\left(\sin \left(\theta_{1}\right) \cos \left(\theta_{2}\right)+\cos \left(\theta_{1}\right) \cos \left(\alpha_{1}\right) \sin \left(\theta_{2}\right)\right) \sin \left(\theta_{3}\right) \sin \left(\alpha_{3}\right)\right. \\
& -\left(-\sin \left(\theta_{1}\right) \sin \left(\theta_{2}\right) \cos \left(\alpha_{2}\right)+\cos \left(\theta_{1}\right) \cos \left(\alpha_{1}\right) \cos \left(\theta_{2}\right) \cos \left(\alpha_{2}\right)\right. \\
& \left.-\cos \left(\theta_{1}\right) \sin \left(\alpha_{1}\right) \sin \left(\alpha_{2}\right)\right) \cos \left(\theta_{3}\right) \sin \left(\alpha_{3}\right)+\left(\sin \left(\theta_{1}\right) \sin \left(\theta_{2}\right) \sin \left(\alpha_{2}\right)\right. \\
& \left.\left.-\cos \left(\theta_{1}\right) \cos \left(\alpha_{1}\right) \cos \left(\theta_{2}\right) \sin \left(\alpha_{2}\right)-\cos \left(\theta_{1}\right) \sin \left(\alpha_{1}\right) \cos \left(\alpha_{2}\right)\right) \cos \left(\alpha_{3}\right)\right) d_{4} \\
& +\left(\sin \left(\theta_{1}\right) \cos \left(\theta_{2}\right)+\cos \left(\theta_{1}\right) \cos \left(\alpha_{1}\right) \sin \left(\theta_{2}\right)\right) a_{3} \cos \left(\theta_{3}\right) \\
& +\left(-\sin \left(\theta_{1}\right) \sin \left(\theta_{2}\right) \cos \left(\alpha_{2}\right)+\cos \left(\theta_{1}\right) \cos \left(\alpha_{1}\right) \cos \left(\theta_{2}\right) \cos \left(\alpha_{2}\right)\right. \\
& \left.-\cos \left(\theta_{1}\right) \sin \left(\alpha_{1}\right) \sin \left(\alpha_{2}\right)\right) a_{3} \sin \left(\theta_{3}\right)+\left(\sin \left(\theta_{1}\right) \sin \left(\theta_{2}\right) \sin \left(\alpha_{2}\right)\right. \\
& \left.-\cos \left(\theta_{1}\right) \cos \left(\alpha_{1}\right) \cos \left(\theta_{2}\right) \sin \left(\alpha_{2}\right)-\cos \left(\theta_{1}\right) \sin \left(\alpha_{1}\right) \cos \left(\alpha_{2}\right)\right) d_{3} \\
& +\sin \left(\theta_{1}\right) a_{2} \cos \left(\theta_{2}\right)+\cos \left(\theta_{1}\right) \cos \left(\alpha_{1}\right) a_{2} \sin \left(\theta_{2}\right)-\cos \left(\theta_{1}\right) \sin \left(\alpha_{1}\right) d_{2} \\
& +a_{1} \sin \left(\theta_{1}\right)
\end{aligned}
$$

This should result in a set of all possible values of $\theta_{1}, \theta_{2}$, and $\theta_{3}$. There may be no possible combinations, in which case the pose is not reachable by the robot. There may be a single set of joint angles, or there may be multiple sets, in which case it will be necessary to determine which one is the most well-suited to the task.

Once all possible sets of the first 3 joint angles have been determined, then the final 3 joint angles are relatively simple to determine. This is done by splitting the transformation matrix, $T$, into two parts. Equation 2.16 shows two transformation matrices, $T_{a}$, which accounts for the first 3 joints, and $T_{b}$, which accounts for the last 3 joints:

$$
\begin{aligned}
& T_{a}=A_{1} A_{2} A_{3}, \\
& T_{b}=A_{4} A_{5} A_{6} .
\end{aligned}
$$

The value of $T$ has been calculated initially (in Equation 2.8). For each set of $\theta_{1}$, $\theta_{2}$, and $\theta_{3}$, we are able to calculate $T_{a}$, using Equation 2.1 to calculate the $A$ matrices. 
This allows us to calculate $T_{b}$, as in Equation 2.17;

$$
T_{b}=\left(T_{a}\right)^{-1} * T .
$$

Given the nature of the wrist point, each of the last 3 joints contributes in a predictable way to the orientation of the tool frame. The values of $\theta_{4}, \theta_{5}$, and $\theta_{6}$ are then able to be determined using Equations 2.18, 2.19, and 2.20:

$$
\begin{gathered}
\theta_{5}=\operatorname{atan} 2\left(\sqrt{\left(T_{b, 31}\right)^{2}+\left(T_{b, 32}\right)^{2}},-T_{b, 33}\right), \\
\theta_{4}=\operatorname{atan} 2\left(\frac{T_{b, 32}}{\sin \left(\theta_{5}\right)}, \frac{T_{b, 31}}{\sin \left(\theta_{5}\right)}\right), \\
\theta_{4}=\operatorname{atan} 2\left(\frac{T_{b, 23}}{\sin \left(\theta_{5}\right)}, \frac{T_{b, 13}}{\sin \left(\theta_{5}\right)}\right) .
\end{gathered}
$$

The result of this calculation is all possible sets of six joint angles that will allow the robot to reach the given tool position and orientation. If multiple possibilities are found, one must be selected. In the case of small movements, a good choice may be the set of joint angles which are closest in value to the angles of the previous pose [3].

\subsection{Calibration}

Calibration is a process in which the robot is moved to known positions, and the robot controller's position information is compared with the known position in order to obtain an estimate of parameter offsets. With calibration, the accuracy of a robot's movement can be substantially enhanced. In this section, the use of calibration and the conventional methods for calibration of a conventional serial robot arm are described.

\subsubsection{Effect of Calibration}

The kinematic equations presented above in Section 2.1 are able to mathematically describe the movement of a robot arm. In practice, there are many factors which can affect the accuracy of the robot pose. Calibration is a process that can reduce some of these factors substantially, and increase the accuracy of the robot, using changes in software rather than physical changes to the robot. 
In order to control a robot arm, a mathematical model of the arm must be used. The accuracy of movements depends on how closely the mathematical model represents the actual motion of the robot arm. The model described in Section2.1 describes a "perfect" robot: joint lengths, offsets, and joint twist all manufactured exactly to specifications, and all joints able to be moved to exact angles, unaffected by any errors in movement. In practice, a robot arm will be affected by all of these things. The calibration process aims to determine how these errors affect robot movement and change the model to better reflect the physical structure of the robot.

In [25], three levels of calibration are identified. Level 1 focuses on joint angles, Level 2 on the entire kinematic model, and Level 3 on the kinematic model as well as other factors. In this thesis, the Level 1 calibration is performed. Part of the Level 2 calibration is also performed in simulation.

\subsubsection{Joint Angle Errors and Link Errors}

Most of the error in a robot's pose is usually found in the joint angle offset values [7]. The joint angle that a robot will record in its controller software is usually inaccurate, by a predictable amount. The error between the actual joint angle and the controllerrecorded joint angle will remain relatively constant, and this value is known as the joint angle offset, $\delta \theta$. Judd and Knasinski [7] found that joint angle offsets account for $90 \%$ of the root-mean-square (RMS) error of a robot's pose. As such, determining the values $\delta \theta$ for each joint can reduce error by approximately $90 \%$, at least in theory.

Link error is similar to joint error in that we are looking for offsets. These are offsets in the remaining DH parameter values: $\alpha, a$, and $d$. These values account for error in manufacturing of the links and deformation over time. This error accounts for about $5 \%$ of the RMS error of a robot's pose [7]. The remaining $5 \%$ is due to non-geometric error, discussed in Subsection 2.2.4. Non-geometric may increase drastically, however, depending on the situation of the robot, and may cause substantially more than the $5 \%$ predicted in $[7]$.

\subsubsection{Repeatability and Accuracy}

In order to describe how well a robot is able to perform tasks, two different measures are used, repeatability and accuracy [4]. The repeatability of a robot describes how closely a robot can return to a taught pose. Repeatability is expressed in units of 
length. A robot is moved to a given pose, this pose is recorded in the controller, and then after some additional task, the robot is returned to this recorded pose. The pose to which the robot returns will be within a certain distance of the initial pose. For example, a robot with a repeatability of $30 \mu \mathrm{m}$ will be able to return to within $30 \mu \mathrm{m}$ of the initial pose. Because the robot is moving to a known pose, repeatability does not depend on the offset errors discussed in Subsection 2.2.2. Many of the tasks that a robot arm is designed to perform are simple and repetitive, for instance, picking up an object in one location and placing it another, a "pick and place" task, or moving its tool along a known path. In these cases, the exact poses needed for the task are taught in advance. Thus, the repeatability is the only value that will affect the performance of these tasks.

The accuracy of a robot describes how closely the robot can move to a new pose in its workspace which has not been taught, but rather computed by the controller using the kinematic model of the robot. An example task would be to move a robot's end effector to a specific point or along a specific path defined in the world coordinate system, without accurately teaching these positions in advance. In order to perform this task, the exact set of joint angles required for each pose in this path must be calculated. These sets of joint angles will depend on accurately knowing the actual values of the DH parameters. As such, offset error in the DH parameters can have a substantial effect on these tasks. In this thesis, accuracy, like repeatability, is expressed in units of length. It is defined as the maximum distance between the desired end effector position and the actual end effector position. A low accuracy value is well-suited to tasks in which the robot moves more autonomously: it is able to determine where it should move to and then make that movement.

If the joint angle errors and link errors were determined perfectly, then the repeatability and accuracy of a robot arm would be equal. The only error remaining in the robot's movement would be nongeometric.

\subsubsection{Nongeometric Error}

As mentioned in Subsection 2.2.2, offsets in joints and link properties account for approximately $95 \%$ of the total RMS error in the system. The remaining $5 \%$ is caused by nongeometric factors. As nongeometric errors are not considered in this analysis, only a brief description of possible sources of error is given here. Nongeometric error can come from many different sources, with each source contributing only minute 
parts of the total error. Nongeometric errors will affect the repeatability of a robot as well as its accuracy. Modeling error that is nongeometric can be more difficult than modeling geometric errors, as it frequently depends on external factors.

Thermal error can have a substantial effect on the repeatability of a robot. Changes in temperature can cause expansion and contraction in links, changing the overall structure of the robot. A robot working in a typical environment will have a "warm up" period as the movement of the robot's motors warms up the nearby links. Leitner et al. [8] analyzed the repeatability of a robot and its warm up period. The warm up period was 500 minutes, and the fully warmed-up robot had a repeatability value of $0.02 \mathrm{~mm}$, a tenfold decrease from the repeatability value obtained when this warm-up period was neglected $(0.2 \mathrm{~mm})$. This is in an environment of overall constant temperature. Some robots must operate in environments with significantly varying temperatures. Reducing thermal error can be done with a temperature sensor and a predictive model, as in [26].

In addition to thermal error, link shape may change due to heavy loads on the robot arm. The structure of a serial robot has several beam-like links in order. A heavy load placed at the end of the arm can cause bending in the links. This deflection will change dynamically as the robot moves [27]. Joints frequently have some backlash. Given the same target angle, a specific joint may arrive at slightly different locations depending on what direction the joint is rotating from. This is caused by backlash, inherent in the construction of the joint. Complex methods exist to model joint backlash [28]. Age may cause wear on robotic components, which can introduce additional error [29].

\subsection{Methods of Calibration}

There are many existing calibration methods. These methods vary in cost, accuracy, complexity, measurement methods, and processing of calibration data. Some known methods are included in this section.

These methods are generally split into two types: measuring the robot's position accurately (Subsection 2.3.3, 2.3.4), or constraining the robot's position in some way (Subsection 2.3.7, 2.3.8, 2.3.9). These are called "open loop" and "closed loop" methods: methods in which the end effector can move freely, and methods in which the end effector is constrained, as if an additional joint were included between the 
end effector and the ground. Both methods require some way of accurately measuring the pose of the robot. These measurements can be difficult to collect and require a trained operator and specific equipment [30].

An open loop method will require collecting a set of pose data: moving the robot to a given pose, recording the controller's joint angles, and recording either complete or partial pose measurements for each pose. A closed loop method will require moving the robot to a set of various poses, all of which satisfy some constraint, and then recording the controller's joint angles at each pose.

\subsubsection{Manual Joint Mastering with Indicators}

This is the simplest method of calibration available. It is a closed loop method in which the joint angles of the robot are constrained. In this method, a certain position of the robot is known, in which some part of a link will align in some way with a part of the next link at a given joint angle. The operator of the robot will move that joint until these indicators are correctly aligned and then record that joint angle. The measured alignment can be dependent on the actual geometry of the link, or can be measured with specific indicator markings applied to the link [25]. The results of this process are very inaccurate compared with more complex calibration methods, as it relies on the operator judging the position of the robot simply by looking, and uses a single pose. The results may be further affected by additional problems, such as indicator marks being painted over, removed, or moved and incorrectly replaced. As such, this is considered a very rough calibration method for work that does not require high accuracy. This method is typically recommended in robot operation manuals [31].

\subsubsection{Manual Joint Mastering with Precise Measurement}

This is a closed loop method which involves moving the robot accurately to a single pose using a constructed jig with dial gauges. This method is also recommended in robot operation manuals [31]. In this method, a jig must be accurately manufactured and attached to the robot arm. Dial gauges on the jig are able to measure small differences in angle, allowing an operator to adjust the robot pose until it matches the desired reference pose. This process allows for greater precision than using indicator marks, as it involves precise measurement of angles rather than having the robot 
operator judge by sight. However, the results are only as precise as the jig itself, so this jig must be manufactured with very high precision. Only a single pose is measured in this method.

\subsubsection{Full Pose Measurement}

In an open loop method, the pose of the robot is measured either fully or partially. Ideally, the pose would be fully measured as this provides the maximum amount of information with which to perform calibration. However, in practice partial measurement methods may be substantially more efficient. Most open loop methods use some form of partial measurement. The full pose of the robot is typically expressed with six variables: three for position and three for orientation. The method described in [32] uses a coordinate measuring machine (CMM) to measure the full pose of the robot. The procedure for measuring both the position and orientation variables involved a precision-manufactured tool attached to the robot end effector with several targets for the CMM to measure. In this case, the targets were five balls attached in a known configuration. Depending on the position of the end effector in the CMM's workspace, three to four of these balls were able to be measured. By measuring the position of all possible balls, the pose of the robot was measured. The ability to precisely measure the entire pose of the robot simplifies the calibration process. Calibration is done by comparing the actual measured pose of the robot with the pose stored in the robot controller over a number of poses. The technique described in [33] uses a CMM to position a calibration board. Calibration is performed by observing this board using a CCD camera mounted on the robot's end effector. A similar technique is used in [34], which is discussed further in Subsection 2.3.4.

The technique described in 35] uses an ultrasonic range sensor. The sensor array used in this case has several sound emitters and several microphones. Distance between these is measured using the time-of-flight of an ultrasonic wave: by measuring the time difference between the emitter sending the ultrasonic wave and the microphone hearing it, distance can be measured accurately. This system must account for fluctuations in temperature and air pressure which can affect the local speed of sound. A complete sensor array is constructed using three microphones and several sound emitters. These sound emitters are placed in known locations in the base frame and also on specific links of the robot in order to measure the pose. 


\subsubsection{Relative Measurements}

Using relative measurements is also an open loop method of calibration. Like using absolute measurements, this technique may measure all or some of the robot's pose variables. However, these pose variables are not measured in the world coordinate system. Instead, each measurement is taken relative to the previous pose measurement. Relative position calibration measures the change in position and orientation between a pose and a reference pose. The technique in [36] uses a ruler to measure position differences. Relative measurements are taken by comparing photos taken of the ruler at different poses as the robot moves. A similar method is described in [37], in which a camera mounted on the robot's end effector measures the position along a precision-manufactured ruler or straight edge. Additionally, a stereo laser system is used to measure the distance from the straightedge to the tool. In [34], both relative position and orientation are measured. This is done using a camera mounted on the robot's end effector and a reference object that can be recognized by analyzing the images from the camera. In this case, the object is a chess board. Another system using relative measurements is described in [38]. This system uses a metallic grid and a laser distance sensor. The laser distance sensor is mounted on the robot and can sense the distance towards the grid plate with high precision. Additionally, the laser allows for the position on the grid plate to be measured, though not with the same high level of accuracy as the distance.

Once the relative pose measurements have been taken, the calibration can be performed using a Jacobian matrix and singular value decomposition (SVD). This process is well described in [39]. The system is represented with Equation 2.21

$$
\Delta x=J_{r e l} \Delta \zeta
$$

where $J_{r e l}$ is the relative Jacobian, described later, and $\Delta x$ is the measure of relative position and orientation. For each point other than Point 1, $\Delta x$ is the $i$ th relative pose (the difference between pose $\mathrm{i}$ and a refence pose), as is shown in Equation 2.22, 


$$
\Delta x=\left[\begin{array}{c}
\Delta x_{i} \\
\Delta y_{i} \\
\Delta z_{i} \\
\Delta w_{i} \\
\Delta p_{i} \\
\Delta r_{i}
\end{array}\right] .
$$

Results of this equation are stacked to produce a single matrix with a single column of length $6(n-1)$ (where $n$ is the total number of poses).

The Jacobian is calculated for all poses. It is composed of partial derivatives measuring the effect of changing any one parameter on the overall equation. There are 24 total partial derivatives for each point (six each for the joint angles, joint twists, link lengths, and link offsets). For any given parameters (for example, the joint angles) the Jacobian is calculated using Equation 2.23 .

$$
J_{\theta}=\left[\begin{array}{llllll}
\frac{\delta x}{\delta \theta_{1}} & \frac{\delta x}{\delta \theta_{2}} & \frac{\delta x}{\delta \theta_{3}} & \frac{\delta x}{\delta \theta_{4}} & \frac{\delta x}{\delta \theta_{5}} & \frac{\delta x}{\delta \theta_{6}} \\
\frac{\delta y}{\delta \theta_{1}} & \frac{\delta y}{\delta \theta_{2}} & \frac{\delta y}{\delta \theta_{3}} & \frac{\delta y}{\delta \theta_{4}} & \frac{\delta y}{\delta \theta_{5}} & \frac{\delta y}{\delta \theta_{6}} \\
\frac{\delta z}{\delta \theta_{1}} & \frac{\delta z}{\delta \theta_{2}} & \frac{\delta z}{\delta \theta_{3}} & \frac{\delta z}{\delta \theta_{4}} & \frac{\delta z}{\delta \theta_{5}} & \frac{\delta z}{\delta \theta_{6}} \\
\frac{\delta w}{\delta \theta_{1}} & \frac{\delta w}{\delta \theta_{2}} & \frac{\delta w}{\delta \theta_{3}} & \frac{\delta w}{\delta \theta_{4}} & \frac{\delta w}{\delta \theta_{5}} & \frac{\delta w}{\delta \theta_{6}} \\
\frac{\delta p}{\delta \theta_{1}} & \frac{\delta p}{\delta \theta_{2}} & \frac{\delta p}{\delta \theta_{3}} & \frac{\delta p}{\delta \theta_{4}} & \frac{\delta p}{\delta \theta_{5}} & \frac{\delta p}{\delta \theta_{6}} \\
\frac{\delta r}{\delta \theta_{1}} & \frac{\delta r}{\delta \theta_{2}} & \frac{\delta r}{\delta \theta_{3}} & \frac{\delta r}{\delta \theta_{4}} & \frac{\delta r}{\delta \theta_{5}} & \frac{\delta r}{\delta \theta_{6}}
\end{array}\right] .
$$

Each of these partial derivatives are solved using computer algebra software. This allows us to calculate the numerical value of the Jacobian directly using the parameter values at any pose, without performing a derivation each time.

The full Jacobian matrix of any pose is:

$$
J_{i}=\left[\begin{array}{llll}
J_{\theta} & J_{\alpha} & J_{a} & J_{d}
\end{array}\right] .
$$


The relative Jacobian of a pose other than Pose 1 is:

$$
J_{r e l, i}=J_{i}-J_{1} .
$$

The full Jacobian matrix is constructed by stacking the relative Jacobian of every pose other than Pose 1. The final size of this matrix is $6(n-1) \times 24$.

In this case, we have more equations than unknowns, and a least squares approximation can be performed with singular value decomposition. The results of this are estimates of all 24 error offsets.

SVD is a technique used to solve a linear least-squares problem [40]. In this case, by taking many pose measurements, we have an overdetermined system, and thus the solution will be a linear least-squares approximation. Functions to perform SVD are frequently included in mathematical software, (such as in Matlab's optimization toolbox). This will take $J_{r e l}$ and decompose into 3 matrices: $U, S$, and $V$. In this way, the pseudo-inverse of $J_{r e l}$ which gives a least-square approximation of this overdetermined system is given by:

$$
J^{+}=(V)\left(S^{-1}\right)\left(U^{T}\right) .
$$

Given that $S$ is a diagonal matrix, the inverse of $S$ can be calculated easily. Each diagonal element in $S^{-1}$ is simply the inverse of that element in $S$. However, values of $J^{+}$that correspond to elements of $S$ that are near zero must be eliminated as they contribute to substantial error [36]. The result of this is an approximation of the inverse of the Jacobian matrix given in Equation 2.21, which allows for a solution to that equation, identifying the errors for each parameter.

\subsubsection{Measurement using Theodolites}

One measuring technique frequently used to determine end-effector position is the use of theodolites. These were originally used as surveying tools as early as the 1500 s, and modern versions allow for high precision measurement [41]. A basic theodolite consists of a telescope mounted in such a way that it can be adjusted in a horizontal or vertical direction, and the angle of the telescope can be very accurately measured. When the telescope is pointed at a distant object, these angles allow one to accurately measure the distance to that object. While typical theodolites used for surveying are 
designed to measure distant objects, the concept can also be used to measure closerrange objects such as the poses of a robot in its workspace. These are automatic theodolites, which do not require manual adjustment. A method is described in [42] in which a combination theodolite and vision system is used to determine the line of sight to a target object on the robot's end effector to measure the pose. The rotation of the theodolite is low resolution, and so must be combined with the attached vision system to provide precise angle measurements. The target object is illuminated in order to be seen easily. In this way, a partial pose measurement is taken. This theodolite and vision system must itself be calibrated in order for precise measurements to be taken [43].

A different method using theodolites is described in [44]. This measurement uses triangulation combined with an interferometer to determine full position measurements. Two theodolites are used, and therefore the intersection of the two lines of sight must be the object. The accuracy of triangulation depends on precision knowledge of the position of each theodolite, thus calibration must be performed on the measurement system as well. The method described in [45] is similar to this, although it uses two automatic laser tracking interferometers.

\subsubsection{Cost of Calibration Systems}

The major driving factor of the cost of calibration is the sensors. The cost of various sensors can vary substantially. The CMM is the most expensive sensor mentioned in this section. An approximate cost for a CMM could range from $\$ 2000$ USD up to $\$ 20000$ USD or higher. These systems are able to very accurately determine position within a certain workspace.

Cameras and laser theodolites are more affordable. An example price for a laser theodolite is $\$ 1000$ USD or lower. Cameras have a very wide range of prices. The two cameras used in this thesis were approximately $\$ 600$ USD each. The measurements taken using these systems are not as accurate as those that are taken with a CMM. They depend highly on how they are used.

The ultrasonic range sensor is the most affordable sensor mentioned here. The cost of an ultrasonic range sensor is around $\$ 30$ USD. The accuracy of these devices can be high, but they are quite limited in what they measure and must be aimed at a specific target. 


\subsubsection{Fixed Pose Closed Loop Methods}

While measuring the position of the end effector in space is very useful for calibration, it is not always possible or efficient to have a full measurement system. Closed loop systems rely on constraining the motion of the robot in some predictable way in space. With this constraint applied, the robot, which on its own is an open kinematic chain, can be treated as a closed kinematic chain. In many cases, motion can be very precisely constrained with mininal sensing equipment when compared with the open loop methods discussed above. Calibration can be performed using many different types of constraints.

The simplest constraint is a completely fixed end point [46]. While this completely restricts the motion of a typical 6 degree-of-freedom robot, it is useful in the calibration of "redundant" serial robots - those with more active joints than there are degrees of freedom in the robot's task space. This can also be used to model two manipulators attached together at the end as a single closed kinematic chain. For a 6 degree-of-freedom robot a less restrictive constraint is required so that many different poses can be selected. One type of constraint is a peg attached to the end effector which must be inserted into various holes in known locations. The system in [47] uses a precision manufactured metal dime with several holes in it. The location of these holes relative to each other is well known because the dime is precision manufactured. This gives several possible positions for the robot, and additionally constrains the orientation, although not completely, as the robot is still able to rotate about the axis of the peg. A similar system is described in [46], in which the robot's motion is described as "opening a door", free to rotate in only one axis. These systems constrain 5 degrees of freedom, leaving the robot free to rotate in one axis only. The system in [48] uses a similar constructed object, a "calibration cube", with many possible holes. However, this system uses a tool with two pegs which must be simultaneously inserted into adjacent holes. This prevents rotation about the peg axis, resulting in a system of many possible poses, each fully constrained (fixed in position and orientation). The method described in [49] uses a much more complex end point constraint. This method uses a constructed hexapod device with six telescoping ballbars, which attaches to the robot's tool with magnetic contacts. This device can be taken apart and rearranged into several configurations, resulting in a total of 72 possible fixed poses of the robot. 


\subsubsection{Closed Loop Methods with Fewer Constraints}

Many closed loop methods have far less restrictive constraints on the robot's pose. These methods may constrain robot motion in as little as one degree of freedom. These constraints may still be used to perform calibration. In [50], a system is used in which the only constraint is point contact with a plane in a known location. This constrains only one degree of freedom. A probe is mounted on the robot's end effector, and the robot is constrained to move such that this probe is touching a precisionmanufactured plane. Another possible method uses multiple planes, as a single plane may not always be enough for a full calibration [51]. A point contact constraint restricts the motion of the robot such that some part of the robot's tool must remain at a given point. However, the orientation can vary. This can be expressed as a ball joint, which allows full rotation but no translation. As such, 3 degrees of freedom are constrained. In [52] and [53], a point contact constraint is used to calibrate a robot.

\subsubsection{Closed Loop Methods with Laser Line}

A common method for constraining the end effector motion is by attaching a laser pointer to the robot, which must always be pointing at one given location. The calibration method used in this thesis features the use of a laser line. The laser dot must be either measured by some device or judged visually by the operator [54]. While judging by eye produces less precise measurements, pointing the laser at a distant object magnifies the effect of orientation errors, so this is more precise that visually judging the robot's joint angles as in Subsection 2.3.1. Using a camera or

other sensor provides much more precise measurements. In [55], a system is proposed that uses a position sensitive detector (PSD) to detect the laser dot.

The advantages of a PSD over other camera types are speed and accuracy compared to cameras of similar cost. However, PSDs can only sense a single dot of light. With the use of a simple laser pointer, use of a PSD is advantageous, however in a situation where a more complex shape must be observed, such as in this thesis, PSDs cannot be used. This is discussed in Chapter 3. In [9], an assembly of 2 PSDs is used to further constrain the motion of the robot. By reflecting the laser line from one PSD to the other and measuring both, a system can be set up in which the laser line orientation must remain constant, and the robot must move only along the laser line. The calibration method performed in this thesis is a similar laser-line based method 
with an assembly of two cameras and a beam splitter to read the laser line, rather than two PSDs. This assembly is able to provide the same constraint on the robot's pose as above, and additionally constrain rotation about the laser line axis. 


\section{Chapter 3}

\section{Experimental Method}

This chapter describes the basic experimental method used for robot calibration in this thesis. A laser is used to provide a linear constraint, similar to methods discussed in Subsection 2.3.9. The laser is attached to the robot's end effector, which is then programmed to point the laser into a box containing an assembly of two cameras, which can read the position and direction of the laser line. These methods are modified in order to fully constrain the end effector orientation. To constrain rotation about the laser axis, line optics are attached to the laser such that it projects a cross shape, rather than simply a dot. By keeping the orientation of this cross constant, rotation will be fully constrained. However, the use of PSDs is no longer possible when sensing a cross shape rather than a dot, as these devices can only sense a single light spot. Hence, an assembly of two cameras is used.

The laser pointer is attached in robot's Tool $\mathrm{Y}$ axis. The line optics are attached to this laser. The position of the laser will follow the position of the robot's end effector.

\subsection{Robots}

This experiment was performed with 3 different robots: two of the same model, FANUC S-420iF [14, shown in Figure 3.1, and one Yaskawa Motoman MH180 [15, shown in Figure 3.2. The properties of these robots are described in Table 3.1. 
Table 3.1: Table of robot properties.

\begin{tabular}{|c|c|c|}
\hline Robot & FANUC S-420iF & Motoman MH180 \\
\hline Maximum Reach (horizontal) & $2.852 \mathrm{~m}$ & $2.702 \mathrm{~m}$ \\
\hline Maximum Reach (vertical, above base) & $3.034 \mathrm{~m}$ & $3.061 \mathrm{~m}$ \\
\hline Maximum Payload & $120 \mathrm{~kg}$ & $180 \mathrm{~kg}$ \\
\hline Repeatability & $\pm 0.4 \mathrm{~mm}$ & $\pm 0.2 \mathrm{~mm}$ \\
\hline
\end{tabular}

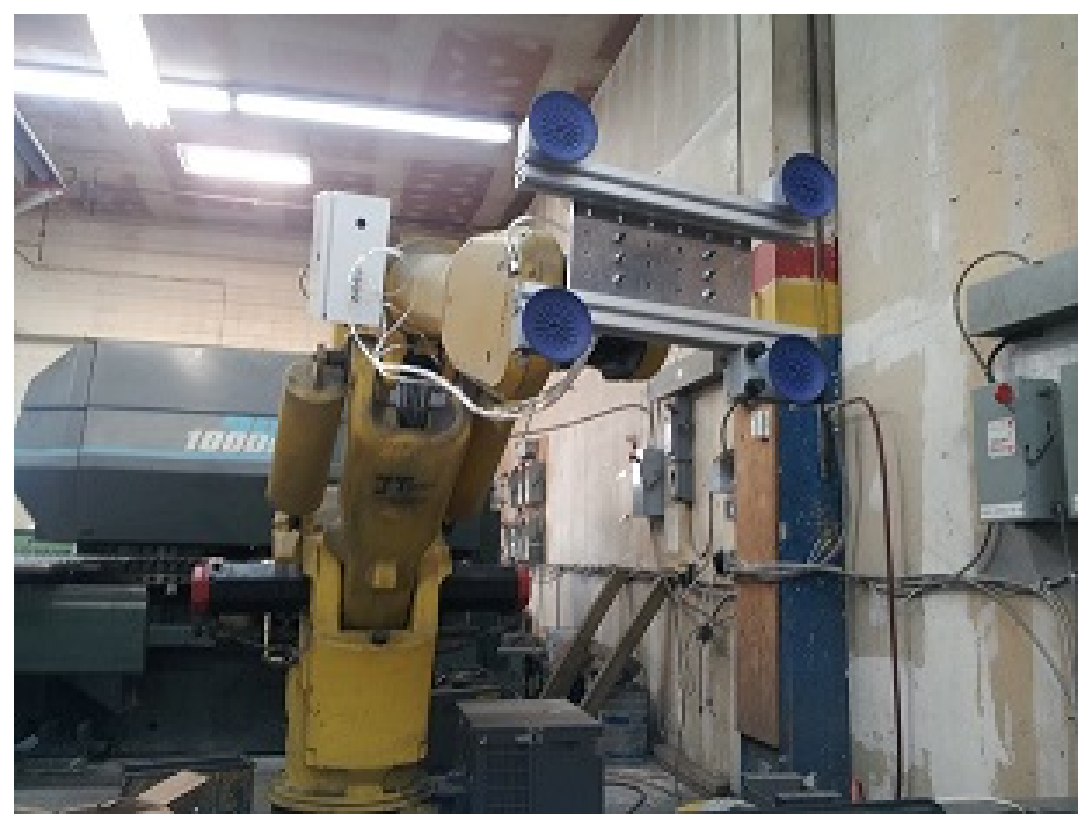

Figure 3.1: Fanuc S-420iF robot arm. 


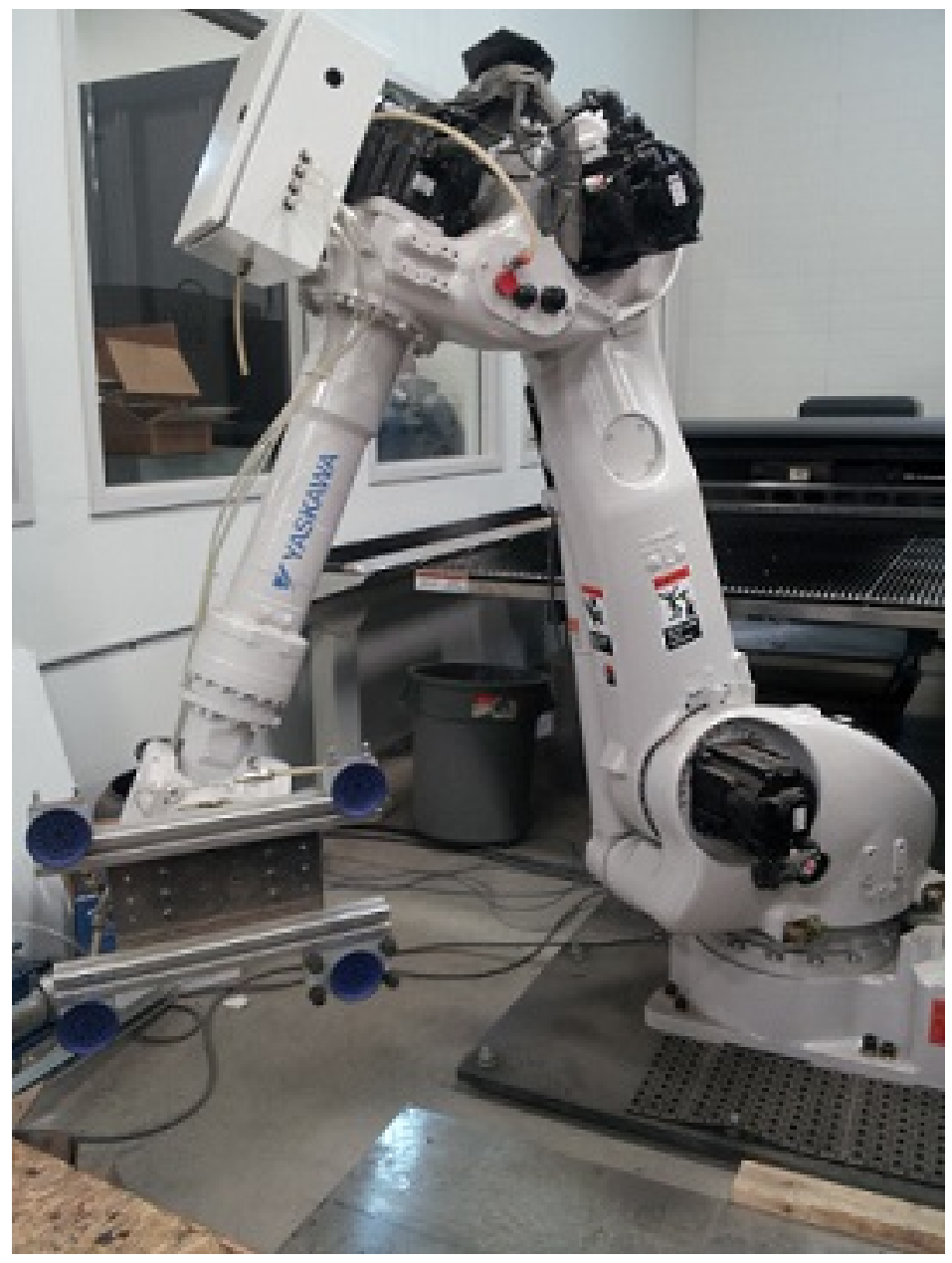

Figure 3.2: Motoman MH-180 robot arm. 


\subsection{Movement of Robot}

During the course of a single calibration, the robot's end effector will be moved along a single straight line. All data points collected will be along this line. The line must be selected such that the parameter errors are observable in the data. The ideal line was selected such that:

- The line is long, crossing a large portion of the robot's workspace.

- There is a significant amount of movement in each joint as the robot progresses along the line.

- The line avoids singularities in the robot's workspace.

Additionally, during the process of simulation the observability of the parameters was verified.

The process of obtaining data points along this line is summarized in Figure 3.3 . which mentions the adjustment process. A sample of this adjustment process is described in Figure 3.4 which lists the steps of a translation adjustment along the Tool $\mathrm{Z}$ axis. 


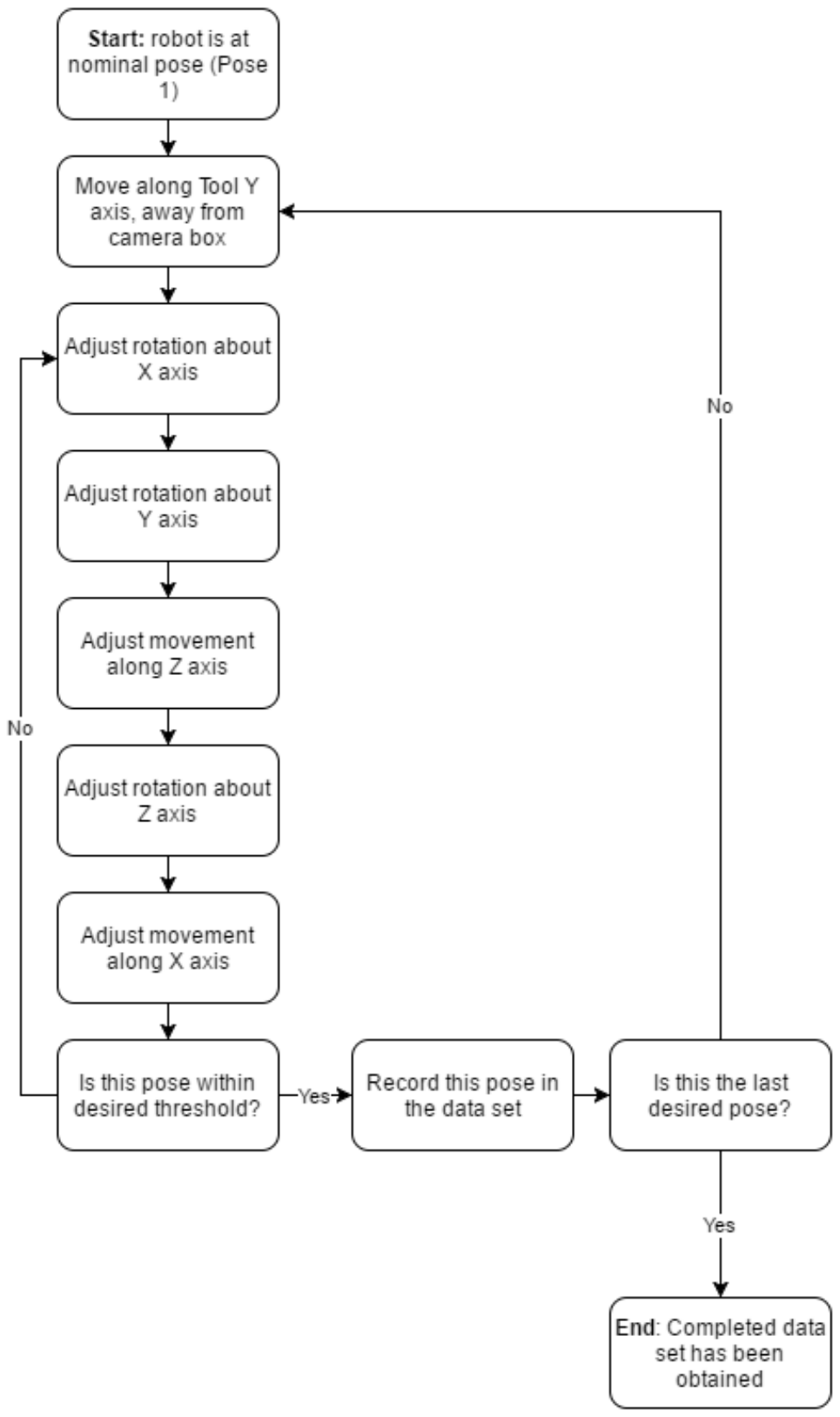

Figure 3.3: Flowchart describing calibration process. 


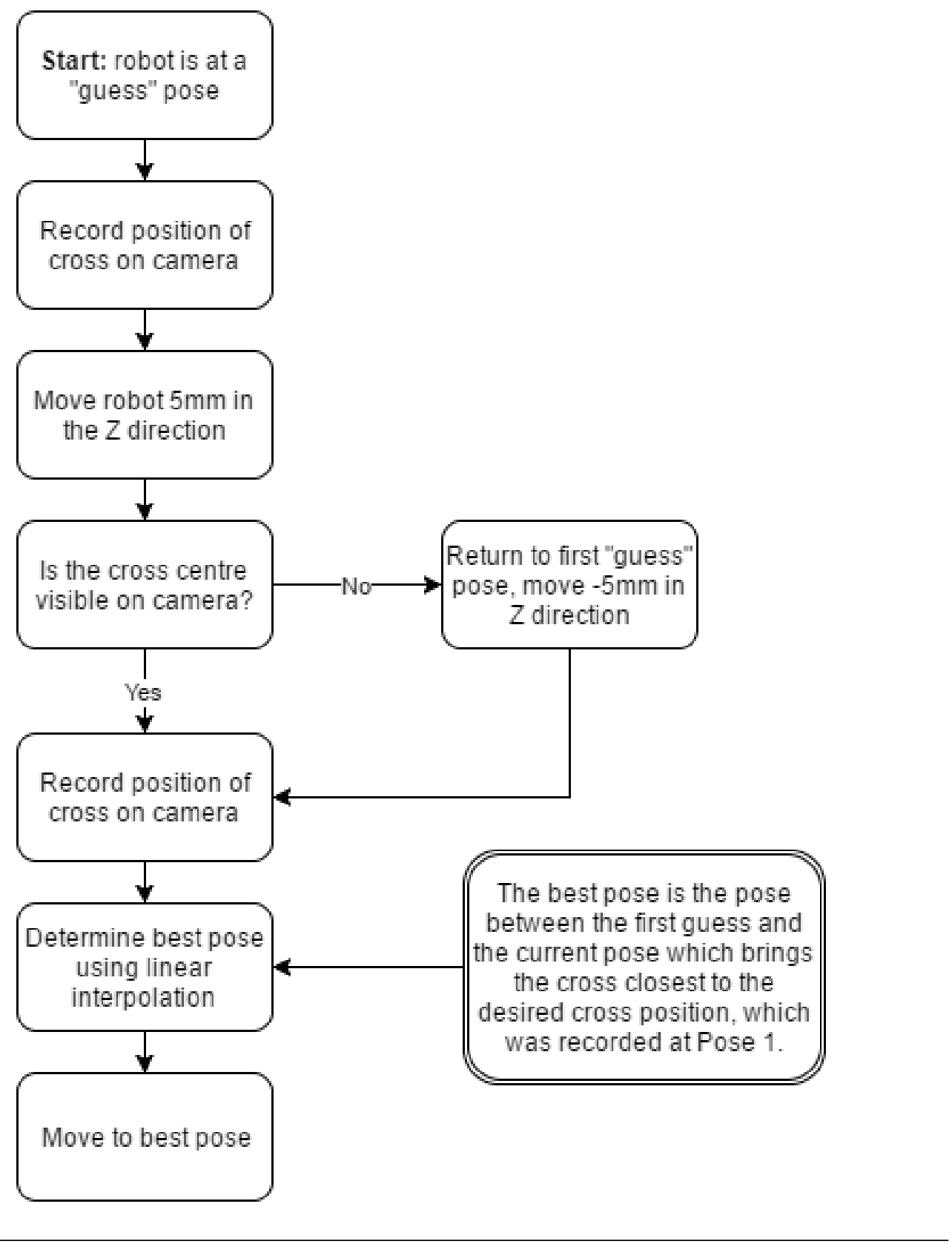

Figure 3.4: Flowchart describing calibration process. 


\subsection{Camera Assembly}

The ultimate goals of the camera assembly are:

1. to measure the location of a point on the laser line;

2. to determine the angle of the laser line in space;

3. to measure the rotation of the robot's end effector about the axis of the laser line.

The goal of the calibration routine will be to adjust robot pose to keep these values as consistent as possible. This will simulate an end-effector constraint in which the end effector orientation must remain completely consistent and the movement of the robot must be along one specific axis: that of the laser line.

\subsubsection{Geometry of Camera Assembly}

Two cameras must be set up in such a way that both can sense the laser line. A beam splitter is used for this purpose. The beam splitter will divide the laser line into two: one beam is transmitted directly in its original direction and the other is reflected. By putting a beam splitter at a 45 degree angle, the original laser cross will be projected in two directions, at 90 degrees from each other.

By placing the two cameras at different distances from the beam splitter, this system will effectively measure two different points on the laser line, which allows us to determine the angle of the laser line in space. The function of this setup is shown in Figure 3.5. The nominal (or desired) laser line is represented as a thicker line. This is a representation of a laser line perfectly orthogonal to the first camera plane. Upon reaching the beam splitter, it splits into two lines, each of which intersects a camera plane directly in the centre. One other line is provided to demonstrate how this camera setup reads different laser lines. With a single camera setup, only a single point on the laser line would be seen. It would be impossible to distinguish two lines at different orientations if they intersected the camera plane at the same point. In this way, the two lines shown in Figure 3.5 would be indistinguishable with only one camera, as they both intersect Camera Plane 1 at the same point. With the addition of a second camera, these lines can be differentiated, as they intersect Camera Plane 2 at different locations. 


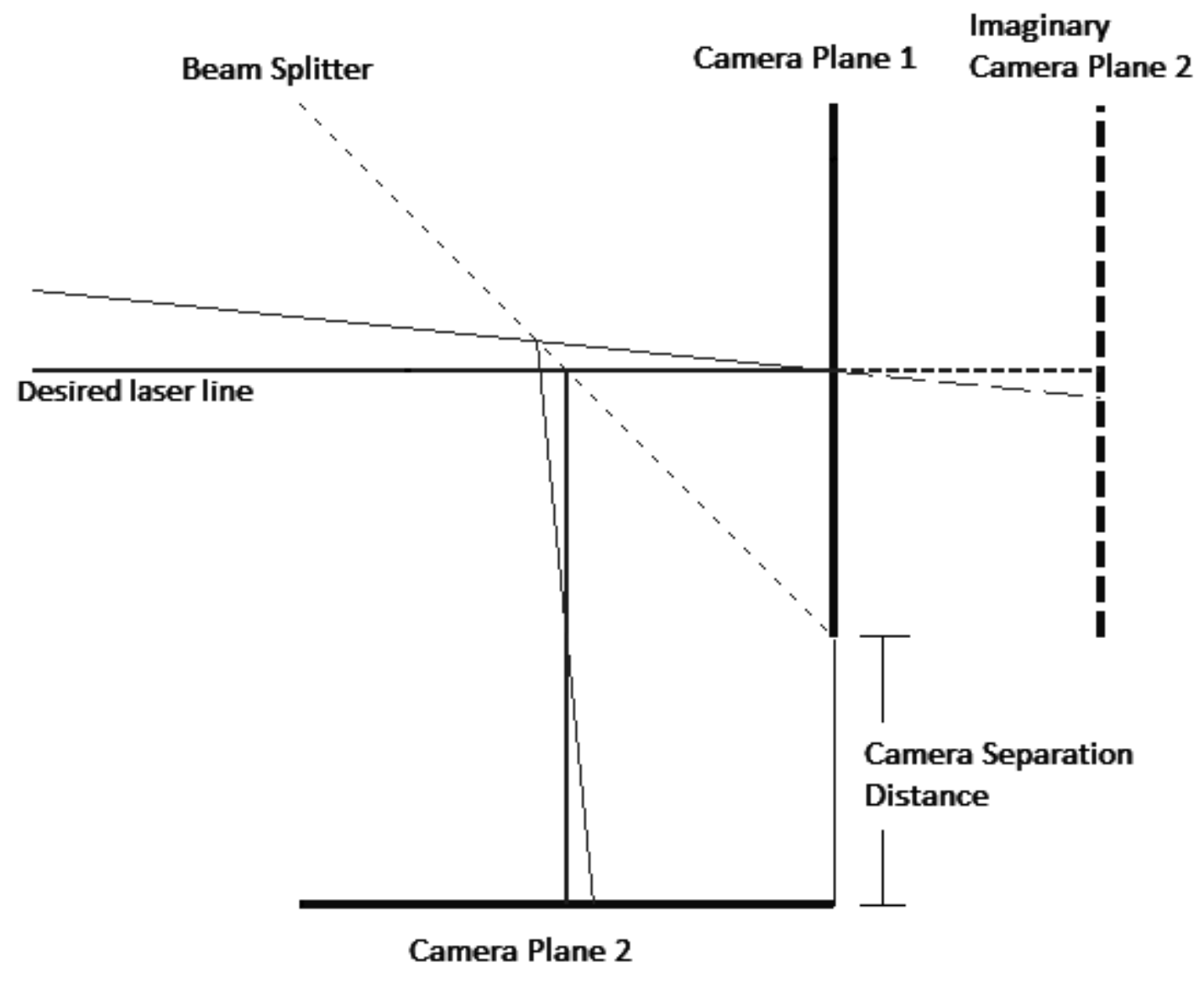

Figure 3.5: Use of beam splitter and two cameras.

As the image reaching the second camera is a direct reflection, this image will be reversed in processing. This reversed image will serve the same function as if an image was taken at the location of the imaginary camera plane. This reflected setup allows us to effectively measure two points along the line. The camera separation distance must be non-zero in this case, so that the two cameras are not measuring the same image. The rotation about the laser axis can be determined simply using the orientation of the cross on the image taken from either camera.

\subsubsection{Physical Setup of Camera Assembly}

The final assembly was constructed to fit inside a sealed metal box, to reduce dust on the sensitive optical components and to reduce ambient light in the images. Figure 


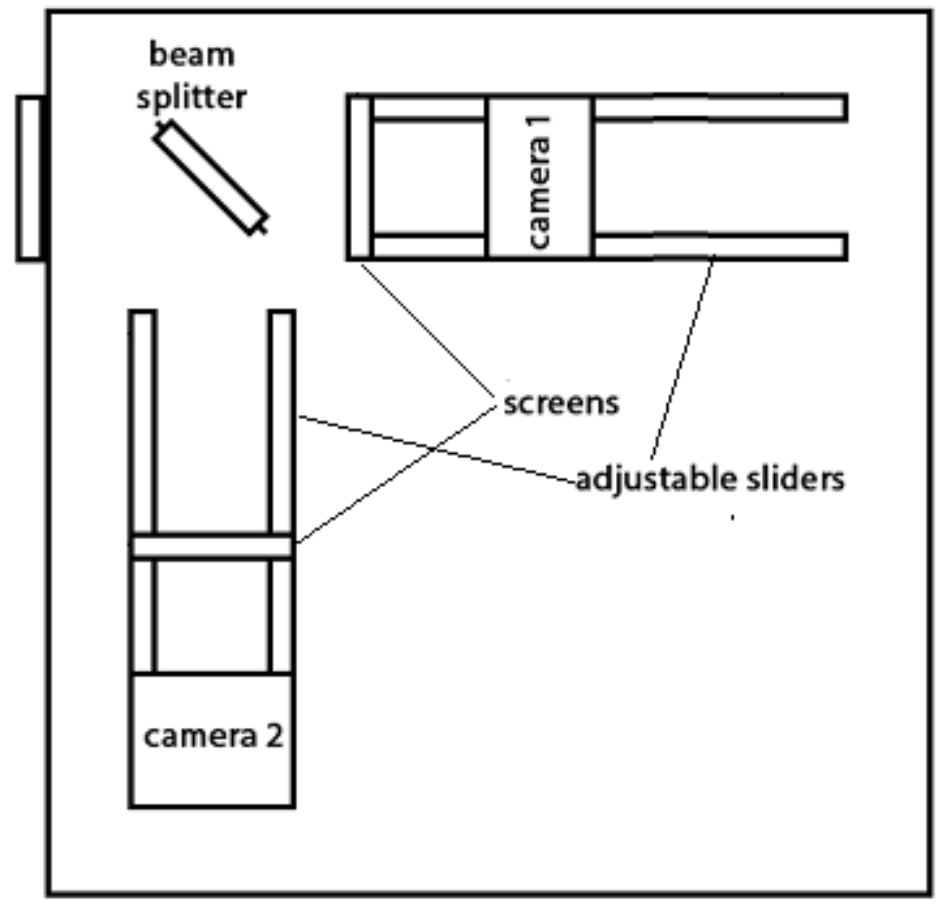

Figure 3.6: Constructed camera assembly.

3.6 gives a top view of the assembled box. Figure 3.7 shows a photo of the completed box.

On the left side of the box is a window through which the beam enters. The beam splitter is placed directly inside this window, at a 45 degree angle. The two cameras are placed in the direction of the laser line. Each camera is mounted such that its distance from the beam splitter can be adjusted through the use of sliders. This allows us to change the camera separation distance (as described previously in Subsection 3.3.1). Allowing the laser to enter the cameras directly does not provide an acceptable image, as too much light enters the camera. To account for this, a screen is mounted to the camera assembly. It is attached such that the distance between the camera and the screen remains constant regardless of the position of the camera. These mylar screens prevent the laser light from entering the camera 


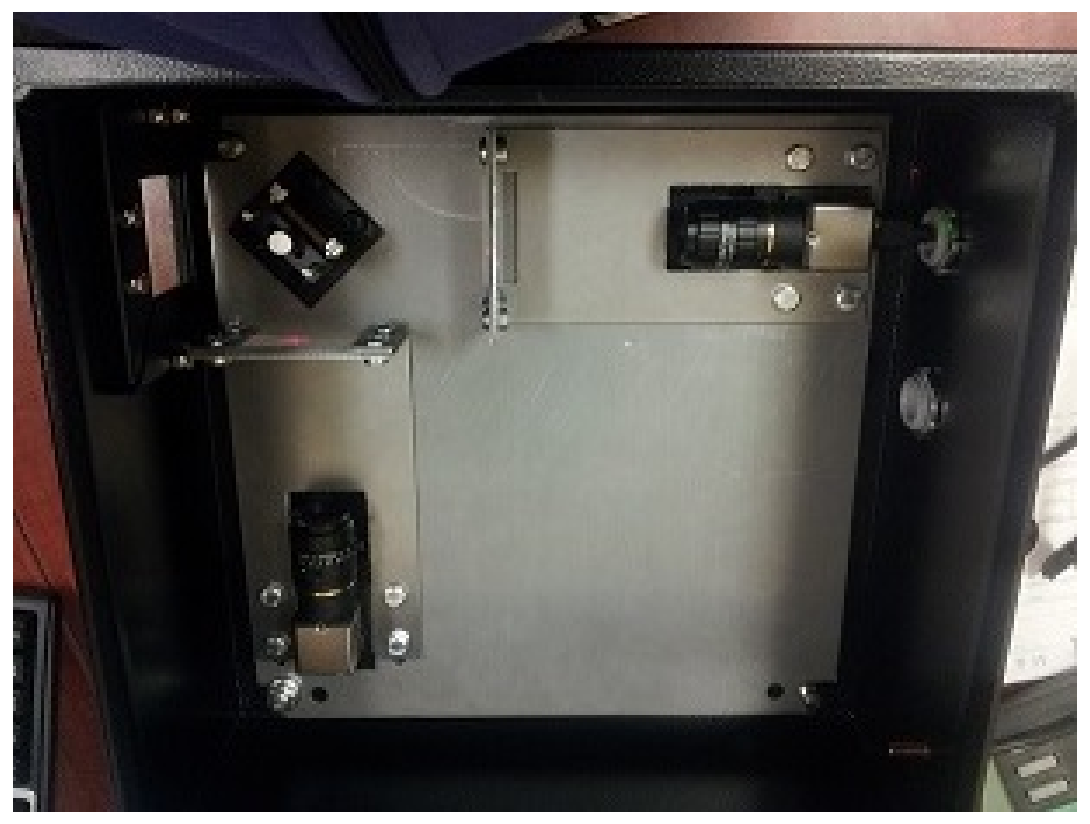

Figure 3.7: Photo of camera assembly.

directly. Instead, the cross is projected on the screen and the camera is taking an image of this projected cross. Mylar is used instead of plain paper to avoid some of the texture of the paper showing up in the images.

The full manufacturer specifications for the cameras are available in [56]. CMOS cameras were chosen over CCD cameras as they provide greater speed for a simple image such as the cross-shape image [57]. No colour is required in the image, so a monochrome camera was selected. A red filter is attached to the camera for ease of sensing the red-coloured laser line. The cameras provide a high resolution image, with a 5 megapixel resolution (1920 pixels vertically and 2560 pixels horizontally). The cameras communicate with the computer over USB.

The focus and aperture settings of the cameras were manually adjusted. The aperture was set such that the cross appears large in the image, but does not bleed outwards into the black space in the rest of the image. The focus settings were set such that the cross appeared somewhat blurred. The cross was visible but the cameras were not focussed perfectly on the mylar sheet. This is because the mylar screen had a visible texture if the camera was focussed on it. This removed the need to filter out this texture in the image processing stage. The cameras were connected with the computer over USB. 


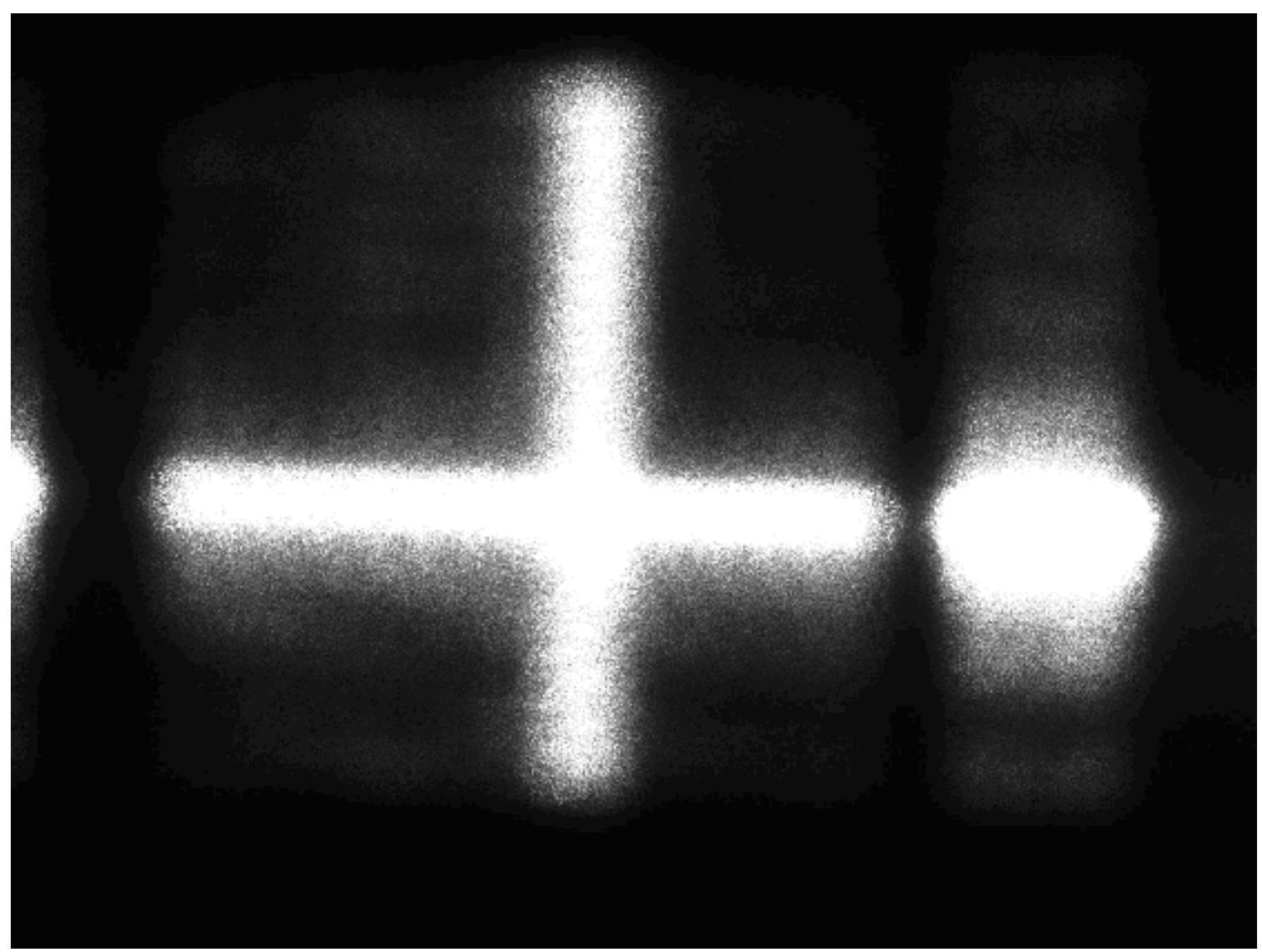

Figure 3.8: Input image.

\subsection{Image Analysis}

This section describes the process of obtaining an image from the cameras and extracting the necessary information from that image. An example of an input image used in this process can be seen in Figure 3.8.

The accuracy of the calibration depends heavily on the accuracy of the image analysis. Because of this, the image analysis is a multi-stage process meant to increase the overall accuracy. The image is given some initial processing, followed by an estimation of the cross centre and angle using Hough transform. Next, this approximation is used to generate regions of interest (ROI), in which a moment algorithm is performed to find the cross centre location and angle. 


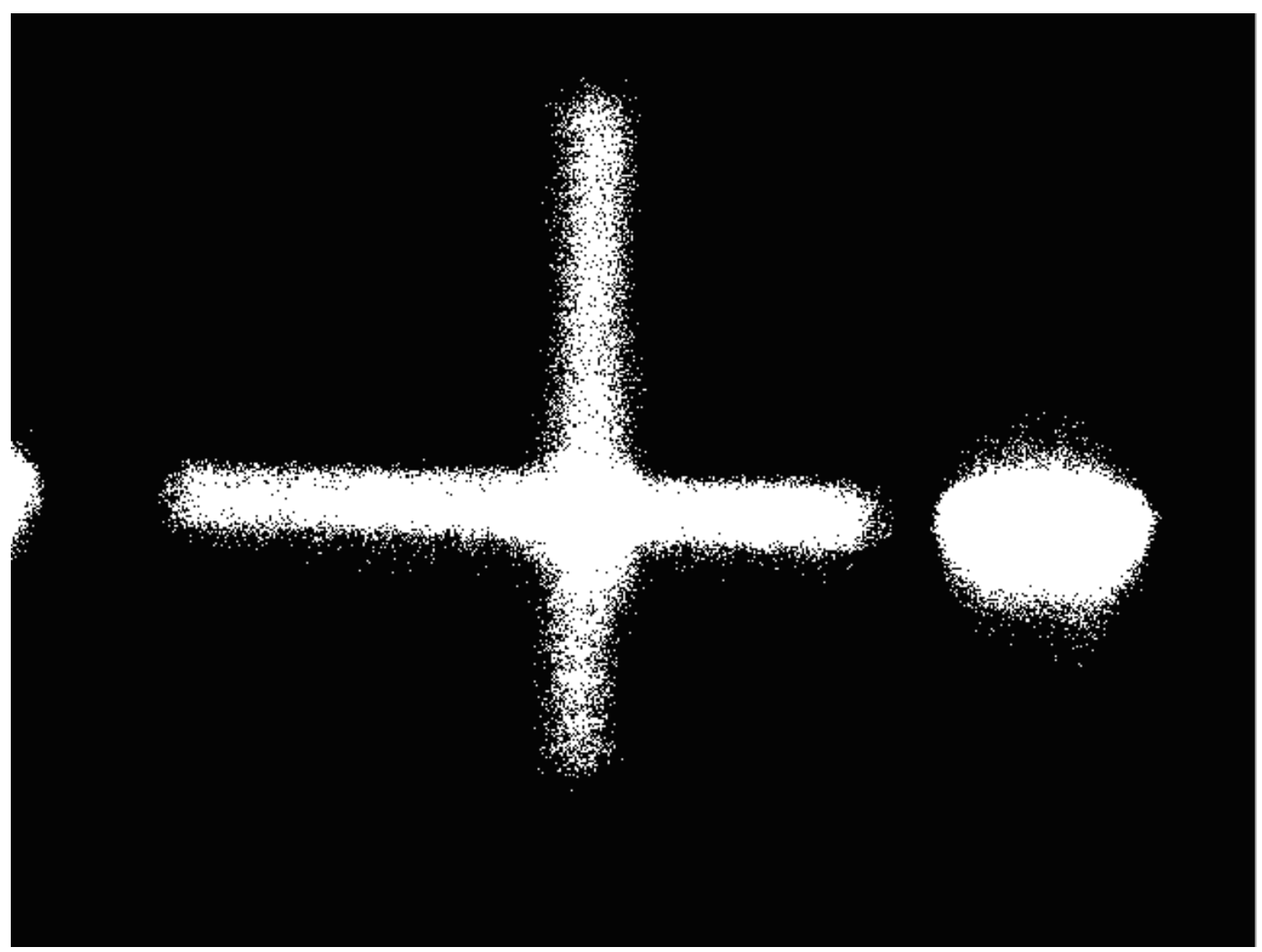

Figure 3.9: Input image converted to black and white.

\subsubsection{Initial Processing}

There are some preparatory steps that must be taken before analyzing the cross positions. First, the image is converted to black and white. The result of this is seen in Figure 3.9 In Figure 3.8, two shapes can be seen on either side that are detached from the main cross shape. These appear only on the images taken with Camera 1, and occur due to the mounting of the beam splitter. The beam splitter is small enough that the edges of the cross can be seen around it on Camera 1. These edges must be removed for analysis. These are simply removed by determining the horizontal location of the outer edges of the central cross. While analyzing the image, everything outside of this horizontal area is ignored (treated as a simple black pixel). 


\subsubsection{Initial Estimate Using Hough Matrix}

An estimate of the cross centre and angles is done using the Hough matrix. This technique was originally created in the 1960s by Paul Hough [58] as a method of identifying straight lines in images. The computational power and memory required to calculate the Hough matrix were restrictive at the time [59], but with today's computers this is trivial. The Hough transform allows us to determine the likeliness of a line in an image at any given location and angle. Later, a different parametrization was created [60], which uses $r$ and $\theta$ rather than $x$ and $y$, which allows for the detection of vertical lines. The value of $\theta$ represents the angle of the line, counterclockwise from the vertical axis. The value of $r$ represents the perpendicular distance between the line and the origin. A more general version of this technique [61] can also be used to detect other shapes. The Hough transform allows to generate a matrix and determine which values of $r$ and $\theta$ are likeliest to contain a line. It results in a two dimensional matrix of values (the Hough matrix), where the highest values represent the most likely places for a line to be, in the image. Figure 3.10 shows the Hough matrix of a black and white cross image similar to Figure 3.9. The white areas of Figure 3.10 represent high values.

Two peaks are located on this graph at approximately 90 degrees apart. These represent an approximation of the two lines making up the cross. They are represented in Figure 3.10 as small circles. By taking these line locations and finding their intersection, an approximation of the cross centre is made. The angle of the nearvertical line is used as an approximation of the cross angle. Because of the thickness of the lines of the cross on the image, further analysis is used to refine the cross centre location detection. The centre and angle values determined using the Hough transform allow us to locate regions of interest for the moment algorithm.

\subsubsection{Finding Regions of Interest (ROI)}

The selection of the regions of interest is shown in Figure 3.11. It shows four ROI, each one enclosing one "arm" of the cross. The centre area is not part of any ROI. 


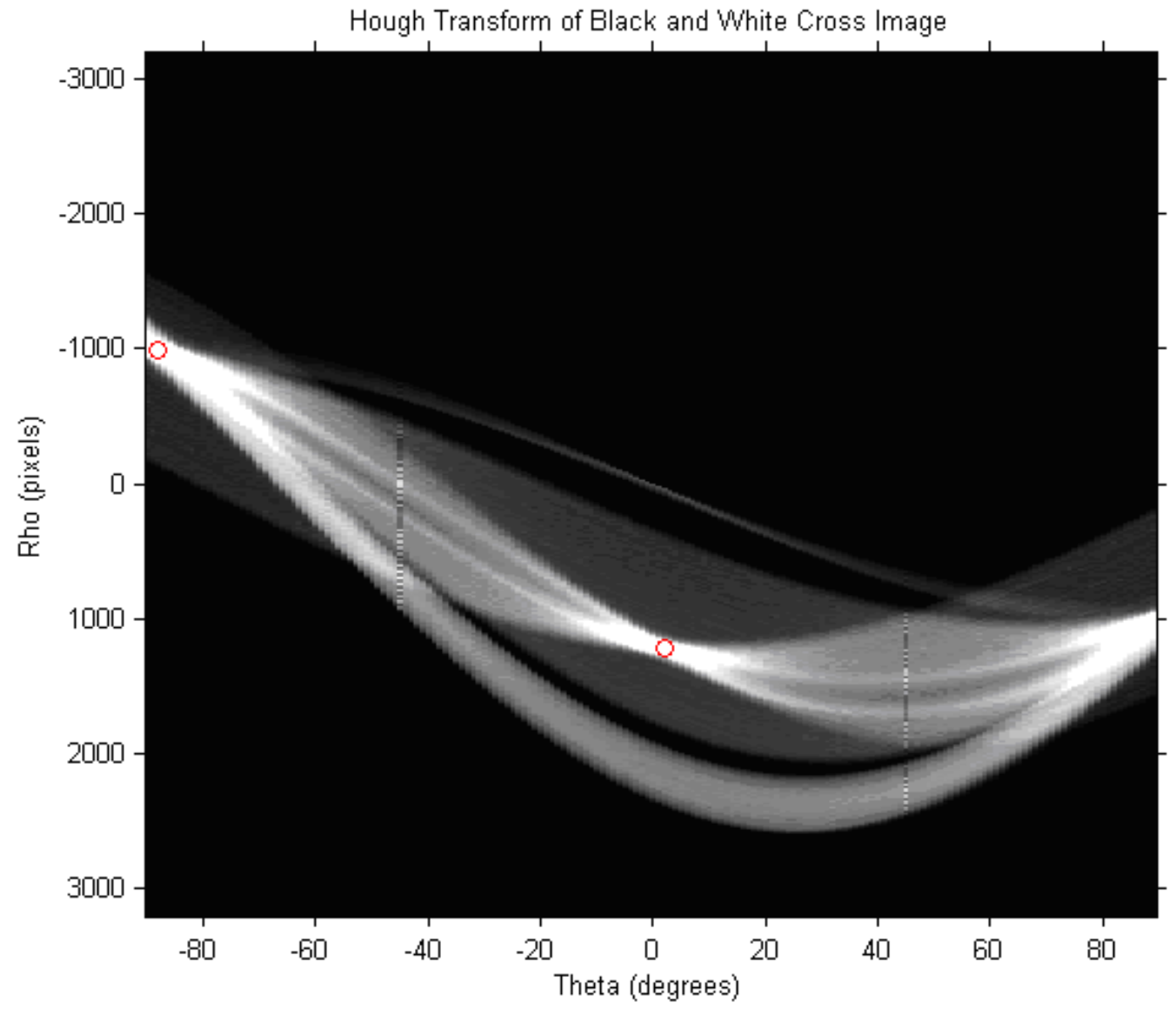

Figure 3.10: Result of hough transform on cross image. 


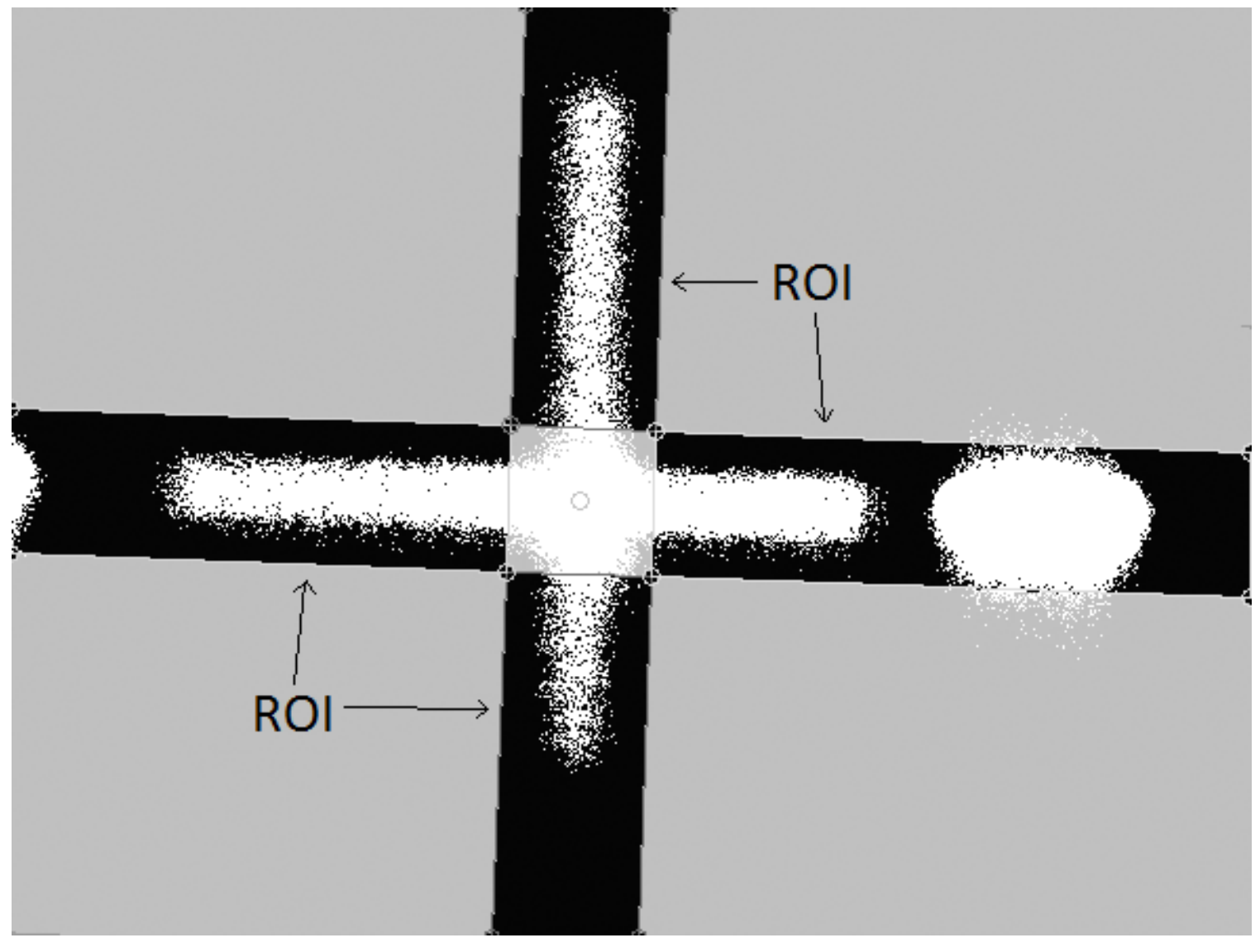

Figure 3.11: Regions of interest on cross image. 
The shape of these ROI is specifically chosen to fulfill the following criteria.

1. None of the centre area of the cross will be included.

2. Each ROI will fully enclose one "arm" of the cross, so that all white pixels of that arm will be included in the analysis.

3. The width of the ROI will not be excessively large, as this increases the time required to perform the moment algorithm.

4. Each ROI is designated as horizontal or vertical, depending on the orientation of the line. In all image analysis cases, the cross orientation only changes minimally, thus during analysis there will always be two approximately horizontal ROI and two approximately vertical ROI.

Note that, as mentioned in Subsection 3.4.1, the white edges detached from the main cross shape will not be included in the analysis. Though they fall within the ROI, they are treated as black pixels.

\subsubsection{Using the Moment Algorithm to Find the Centre and Angle to Sub-Pixel Precision}

The moment algorithm found in 62$]$ is used here. This algorithm works by calculating a $y$ value for each column of pixels at a specific $x$ value. This $y$ value is found using Equation 3.1 .

$$
\frac{\sum_{y=1}^{y_{\max }} y \cdot I^{p}(x, y)}{\sum_{y=1}^{y_{\max }} I^{p}(x, y)} .
$$

Where $I(x, y)$ represents the intensity at the location $(x, y)$. The variable $p$ is used as a power factor which allows for some control over the function depending on the use. Here, the value of $p$ remains at 1 .

This is performed over the two horizontal ROI. The vertical ROI are evaluated with the same equation, with the $x$ and $y$ switched. This equation was found to be slightly inaccurate at the far edges of the ROI, so a total of 50 points are removed from each end. The remainder of the two horizontal ROI are merged into one. The vertical ROI are similarly merged. This results in two data sets: one set of points representing the horizontal portion of the cross, and one representing the vertical. 
This can be seen in Figure 3.12. In this, the thicker lines represent the points used to calculate the best fit line. The darker portions of these data sets are the 50 discarded points.

The 50 points removed from the edge did not have reliably accurate results. Points near to the cross centre could be affected by light from the centre square. This effect was not noticeable with a perfectly vertical and horizontal cross, but problematic when the cross was more diagonal. Near the outer edge of the cross, there were very few pixels in each row, so the moment algorithm did not reliably obtain points along the centre of the line.

The final line locations are determined using a simple best-fit calculation on each. (The vertical line is rotated 90 degrees before the best fit calculation is performed as best fit does not work well with near vertical lines). This gives two straight lines on the image. These are also shown over the data sets in Figure 3.12. The final cross centre location is given by the intersection of these lines. 


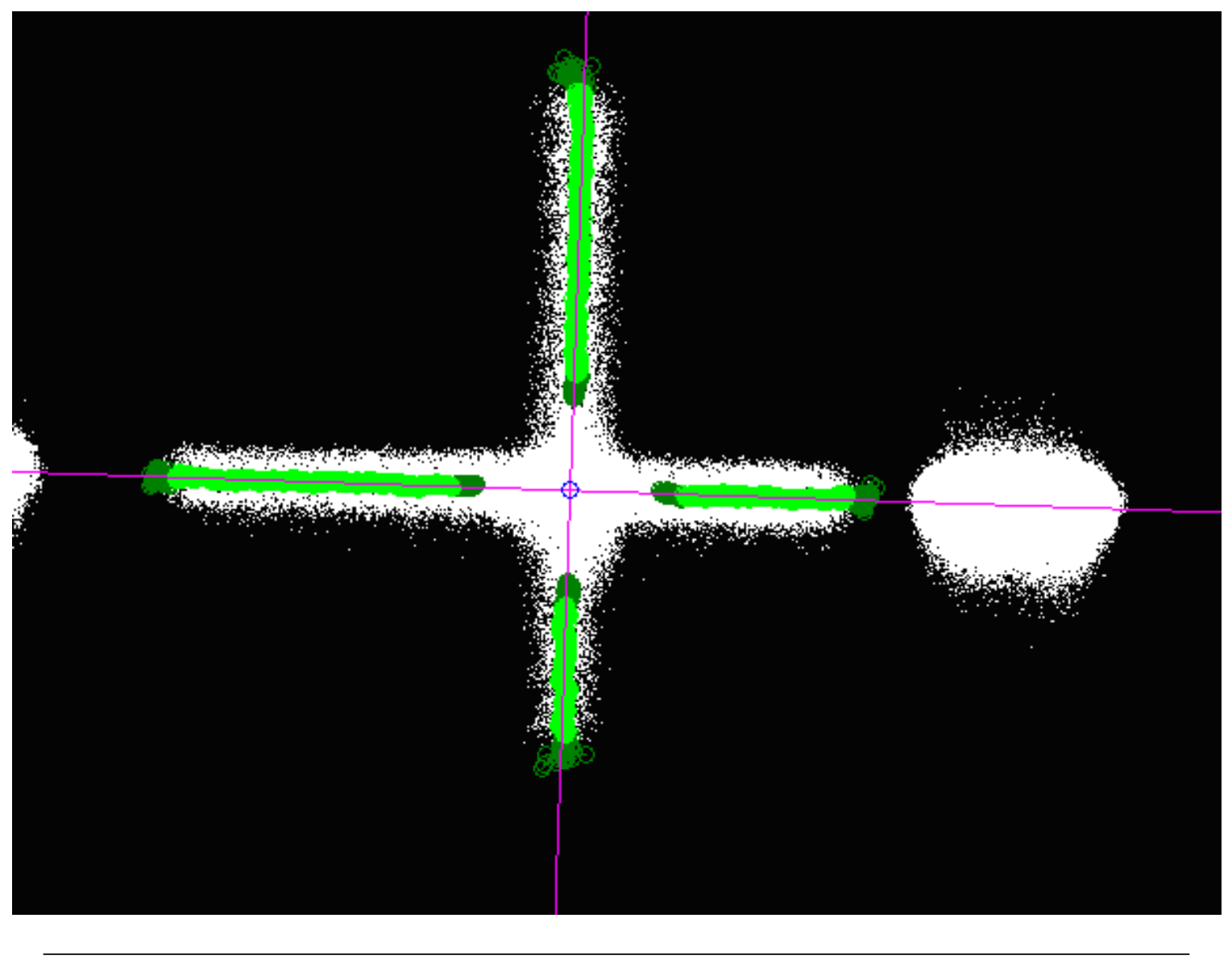

Figure 3.12: Final cross centre determination. 


\section{Chapter 4}

\section{Simulation of Calibration Procedure}

\subsection{Purpose of Simulation}

Two simulations were created before the experiment was performed. The basic simulation was made in order to verify the implementation of the calibration algorithm. The calibration algorithm was tested on a virtually generated data set in which the parameter offset errors were known.

The second simulation, the complex simulation, was made to design the movement process of the robot. During the actual experiment, the robot would follow a specific movement pattern in order to obtain valid data points. The second simulation fully models the positions of the robot as it follows this process.

\subsection{Basic Simulation}

In this simulation, measurement error and noise are not considered. The sample data set which is generated and analyzed is an ideal example. The only errors in this model are the offsets in the $24 \mathrm{DH}$ parameters.

\subsubsection{Creating a Simulated Data Set}

The system we are simulating here is able to measure and constrain the motion of the robot such that the following conditions are met.

1. The orientation of the end effector remains constant.

2. The movement of the robot remains along a single line (in this case the Tool Y axis). 
In this simulation, the process of moving the robot arm to positions that meet these conditions is omitted. Since the simulation relies on synthetic parameter errors, a set of robot joint angle data that is known to be correct is generated first.

The process for determining this data set starts by choosing a single line in space which will be the nominal position of the laser line. First, a set of points along this line in space is determined. Then, using the robot's inverse kinematics, each point can then be represented by a set of joint angles. This line is generated using the "real" values of the robot's DH parameters. The selected line for this simulation spans a large amount of the workspace. The properties of this line are given in Table 4.1.

Table 4.1: Properties of simulation line.

\begin{tabular}{|l|l|}
\hline Length: & $4030.4 \mathrm{~mm}$ \\
\hline Start Point: & {$[2500,-510,-330]$} \\
\hline End Point: & {$[-62.9,2288.9,1027.3]$} \\
\hline Orientation: & {$[0.3665,-0.3491,0.6109](\mathrm{W}, \mathrm{P}, \mathrm{R}$ in $\mathrm{rad})$} \\
\hline Total Points: & 70 \\
\hline
\end{tabular}

The final simulated error data is generated by adding synthetic error values to all of the robot's actual DH parameters. This includes each set of joint angles for each point, as well as the nominal $a, d$, and $\alpha$ values. These synthetic error values were selected roughly using Subsection 2.2.2, in which it is mentioned that joint angle offset errors account for approximately $90 \%$ of total error and link errors for $5 \%$. The remaining $5 \%$ is due to non-geometric error, which is discussed in Subsection 2.2.4. The synthetic error values are given in Table 4.2

Table 4.2: Synthetic error added to DH parameters.

\begin{tabular}{|l|l|l|l|l|l|l|}
\hline Link & 1 & 2 & 3 & 4 & 5 & 6 \\
\hline$\delta \theta$ (degrees) & 0.2 & 0.35 & 0.8 & -0.7 & 0.2 & 0.1 \\
\hline$\delta \alpha$ (degrees) & 0.01 & 0.02 & -0.01 & 0.01 & -0.02 & 0.03 \\
\hline$\delta a(\mathrm{~mm})$ & 0.02 & 0.06 & -0.04 & 0.02 & 0.01 & -0.01 \\
\hline$\delta d(\mathrm{~mm})$ & 0.01 & 0.01 & 0.02 & -0.04 & 0.01 & -0.01 \\
\hline
\end{tabular}


The values of these synthetic errors were assigned such that the error in joint angle was between -1 and 1 degree. This approximate value was chosed based on the value of several similar experiments such as [34]. The error in link twist was assigned to be less than $10 \%$ of this value. The error in link length and offset were set to be quite low, as these depend on the manufacturing of the robot.

\subsubsection{Determining Joint Angle offsets}

The initial correction of joint angle offsets is made using the technique described in [9]. At each point other than the first point, the transformation matrix which represents this position can be written as in Equation 4.1.

$$
T=\left[\begin{array}{cccc}
T_{11} & T_{12} & T_{13} & T_{14} \\
T_{21} & T_{22} & T_{23} & T_{24} \\
T_{31} & T_{32} & T_{33} & T_{34} \\
0 & 0 & 0 & 1
\end{array}\right]
$$

At each point $j$ other than the first point, $\psi$ values are given in Equations 4.2 to 4.5. These equations are modified from those in [9] in order to measure a laser line in the Tool $\mathrm{Y}$ axis. These equations compare values for pose $j$ against the reference pose, pose 1 .

$$
\begin{gathered}
\psi_{j 1}=\left(T_{12}\right)_{j}-\left(T_{12}\right)_{1}, \\
\psi_{j 2}=\left(T_{22}\right)_{j}-\left(T_{22}\right)_{1}, \\
\psi_{j 3}=\frac{\left(T_{12}\right)_{1}}{\left(T_{22}\right)_{1}}-\frac{\left(T_{14}\right)_{j}-\left(T_{14}\right)_{1}}{\left(T_{24}\right)_{j}-\left(T_{24}\right)_{1}}, \\
\psi_{j 4}=\frac{\left(T_{22}\right)_{1}}{\left(T_{32}\right)_{1}}-\frac{\left(T_{24}\right)_{j}-\left(T_{24}\right)_{1}}{\left(T_{34}\right)_{j}-\left(T_{34}\right)_{1}} .
\end{gathered}
$$

A function is created that solves for all of these values given joint angle offset errors. These four $\psi$ values are essentially a measure of how much the offset "guesses" which are given to the function differ from the actual offsets of the system (in this case, these are the synthetic offsets that were specified earlier). Hence, the offset 
values we are looking for are the values that minimize the output of this function.

This five-parameter optimization is performed using the function "lsqnonlin.m", a part of MATLAB's optimization toolbox. The result of this is the five offset angles for

joints two through six. Once the initial estimates of joint angle offset are determined, the nominal angles are modified to include these offset errors.

\subsubsection{Determining Link Twist offsets}

The link twist offsets can be determined using a modified form of the technique used to determine joint offsets. The modifications to the technique are as follows.

1. The rotation about the laser line axis is assumed to be zero. The position data is generated in this simulation such that this is true, but the technique from $[9$ uses data in which this rotation is not controlled.

2. Direction of movement along the line is not considered.

By considering only orientation in the determination of these factors, dependence on errors in link length and link offset is eliminated. The offset errors in angular DH parameters can now be determined with greater accuracy. Equations 4.2 to 4.5 are replaced with Equations 4.6 to 4.8 to meet these conditions:

$$
\begin{aligned}
& \psi_{j 1}=\left(T_{11}\right)_{j}-\left(T_{11}\right)_{1}, \\
& \psi_{j 2}=\left(T_{22}\right)_{j}-\left(T_{22}\right)_{1}, \\
& \psi_{j 3}=\left(T_{33}\right)_{j}-\left(T_{33}\right)_{1} .
\end{aligned}
$$

Optimization is performed as above using these modified equations. The result of this optimization will be an offset estimate of ten parameters: The joint twist values for joints one through five and further refinement of the joint angle offsets for joints two through six. The offset errors determined here are added to the nominal DH parameters to determine the estimate of the actual DH parameters. 
Table 4.3: Joint angle offset determination.

\begin{tabular}{|l|l|l|l|}
\hline & Simulated Error $(\mathrm{deg})$ & Identified Error $(\mathrm{deg})$ & Error $(\%)$ \\
\hline$\delta \theta_{2}$ & 0.35 & 0.33 & -4.50 \\
\hline$\delta \theta_{3}$ & 0.8 & 0.84 & 5.42 \\
\hline$\delta \theta_{4}$ & -0.7 & -0.77 & 10.25 \\
\hline$\delta \theta_{5}$ & 0.2 & 0.16 & -21.77 \\
\hline$\delta \theta_{6}$ & 0.1 & 0.09 & -10.62 \\
\hline
\end{tabular}

\subsubsection{Results of the Simulated Calibration Procedure}

The result of the initial determination of joint angle offsets (Subsection 4.2.2) is listed in Table 4.3. The result of the second calibration procedure (Subsection 4.2.3) for the Link Twist offsets is listed in Table 4.4. The second calibration procedure also refines the estimates of Joint Angle offset. These refined results are listed in Table 4.5. The identified error is listed to only four decimal places, so the identified error in Table 4.4 appears identical to the simulated error due to the fact that the differences are insignificantly small.

Table 4.4: Joint twist offset determination.

\begin{tabular}{|l|l|l|l|}
\hline & Simulated Error (deg) & Identified Error (deg) & Error $(\%)$ \\
\hline$\delta \alpha_{1}$ & 0.01 & 0.01 & $1.11 \times 10^{-7}$ \\
\hline$\delta \alpha_{2}$ & 0.02 & 0.02 & $-9.36 \times 10^{-9}$ \\
\hline$\delta \alpha_{3}$ & -0.01 & -0.01 & $-2.82 \times 10^{-8}$ \\
\hline$\delta \alpha_{4}$ & 0.01 & 0.01 & $2.92 \times 10^{-7}$ \\
\hline$\delta \alpha_{5}$ & -0.02 & -0.02 & $-1.03 \times 10^{-7}$ \\
\hline
\end{tabular}

\subsection{Simulation of Data Acquisition Process}

The purpose of this simulation is to mimic the process of actually obtaining data from a real robot. This includes the movement of the robot, the placement of the CMOS 
Table 4.5: Refined joint angle offset determination.

\begin{tabular}{|l|l|l|l|}
\hline & Simulated Error $(\mathrm{deg})$ & Identified Error $(\mathrm{deg})$ & Error $(\%)$ \\
\hline$\delta \theta_{2}$ & 0.35 & 0.33 & -4.50 \\
\hline$\delta \theta_{3}$ & 0.8 & 0.82 & 1.97 \\
\hline$\delta \theta_{4}$ & -0.7 & -0.70 & $4.43 \times 10^{-9}$ \\
\hline$\delta \theta_{5}$ & 0.2 & 0.20 & $-2.95 \times 10^{-8}$ \\
\hline$\delta \theta_{6}$ & 0.1 & 0.08 & -28.55 \\
\hline
\end{tabular}

cameras, and the feedback they would give from the laser line. This simulation method provides several advantages: it allows for a complete simulation of the required robot movement functions (to facilitate the use of this process in a real-world experiment) and it allows us to analyze the effect of errors in movement and image acquisition on the final process. During this process, an initial "nominal" position of the robot is selected. From this position, the position of the camera planes in space are designated. Following this, each additional point in the simulated data set is calculated by moving to an estimated position (determined using the robot controller) and then performing the adjustment steps described in Subsections 4.3 .2 and 4.3 .3 .

\subsubsection{Position of Camera Plane and Obtaining Camera Feed- back}

In the physical environment in which the experiment is conducted, an assembly with two cameras is placed near the robot. In this simulation, the virtual location and orientation of the camera assembly is recorded in world frame coordinates. The feedback of the cameras is then determined computationally. The camera assembly is represented as two parallel camera planes, with a designated separation distance. This is representative of the actual camera plane orientation with the second camera plane mirrored, as seen in Figure 3.5. The position and orientation of the first camera plane gives a new coordinate reference frame (referred to as the "camera frame" $(x, y, z)$.

The origin of the camera frame is where the centre of the camera's sensor would be. The position of the camera plane can be arbitrary in space (although it must be positioned such that the robot's tool could point the laser cross into it). In this simulation, the camera plane is selected based on the desired robot trajectory. A 
nominal position of the robot is used to designate the camera plane. The nominal position is the start of the calibration process. At this nominal position, the laser line intersects with the origin of both camera planes and is normal to them. The camera frame orientation is the same as the robot's nominal orientation. The camera frame origin is also along the tool Y-axis in the nominal position. Thus, the transformation matrix from the tool frame in the nominal pose to the camera frame is a simple $\mathrm{Y}$-axis translation, as given in Equation 4.9, where $T$ is the transformation from world frame to tool frame in the nominal position and $d$ is an arbitrary distance along the Y-axis, representing the distance from the tool frame origin to the camera plane:

$$
T_{\text {camera }}=T\left[\begin{array}{llll}
1 & 0 & 0 & 0 \\
0 & 1 & 0 & d \\
0 & 0 & 1 & 0 \\
0 & 0 & 0 & 1
\end{array}\right] .
$$

Once this frame is defined, the camera feedback for any given robot position can be obtained by finding the intersection of the tool $\mathrm{Y}$ axis at that position with the camera frame $x-z$ plane, and expressing this point in camera frame coordinates. This will give the distance of the laser dot from the centre of the camera plane in camera $x$ and $z$ coordinates.

\subsubsection{Adjusting Pose Using Translation in the Tool Frame}

The result of this process will effectively "move" the simulated robot along a given Tool axis to get the camera's feedback to be as close to the nominal camera feedback as possible. Nominal camera feedback is defined as the camera reading at an initial, nominal pose. The Tool X-Z plane is approximately parallel to the camera plane. Only feedback from the first camera is used here. The current simulated robot position is a "guess" position, calculated by determining a point that would be along the Tool Y-axis according to the nominal DH parameters. The algorithm steps are:

1. the laser position on Camera 1 is determined;

2. a second pose is found, representing a tool movement of a certain small distance along whichever axis is currently being adjusted; 
3. the laser position on Camera 1 is determined for this pose;

4. using linear interpolation, the point between these two poses at which the laser spot on the camera is closest to its nominal position is determined;

5. this pose is recorded as the final adjusted pose.

For all further adjustment steps, this adjusted pose becomes the new "guess" pose.

\subsubsection{Adjusting Pose Using Rotation in the Tool Frame}

While the positional changes are measured using the feedback of one camera, the rotational differences must be measured using both cameras. In order to find a point that is closest to the nominal orientation, differences in feedback from the two cameras are used. Similar to the translation adjustment, the current "guess" pose will be modified in order to reduce the error in orientation. The following steps are used:

1. the current laser position is found for both cameras;

2. the difference between these two positions is calculated;

3. a second pose is found, representing a small tool axis rotation about whichever axis is being adjusted;

4. the laser positions are found for both cameras at this position;

5. the desired point is the point at which the difference between the laser positions on both cameras is the smallest. Using the differences determined at each adjustment pose, and linear interpolation, the pose that is closest in orientation to the nominal pose is found;

6. this pose is recorded as the final adjusted pose.

For all further adjustment steps, this pose becomes the new "guess" pose.

\subsubsection{Full Adjustment at any Pose}

The full adjustment at any pose is done here using the processes described in Subsections 4.3 .2 and 4.3 .3 repeatedly, in both the Tool X- and Z-axes. First, the orientation 
of the tool is adjusted, by performing a Tool $\mathrm{X}$ rotation adjustment, then Tool Z rotation adjustment, and repeatedly performing these two until the difference between camera feedback between each camera is within a specified threshold of the nominal difference.

Following this, the pose of the tool is adjusted, by performing a Tool X translation adjustment, followed by a Tool $\mathrm{Z}$ translation adjustment. This is repeated until the feedback of the first camera is within a certain threshold from the nominal feedback of the first camera.

If, after a certain number of attempts, no acceptable pose was reached, the pose is not recorded. However, the simulation never encountered a pose for which this was necessary. Once an acceptable set of poses is determined using this method, the calibration is performed with this data set in the same way as in Subsection 4.2.2.

\subsubsection{Results of Calibration Using Data Generated with Sim- ulation}

The purpose of this simulation is to model the actual movement of the robot in space in order to prove that the adjustment pattern calibrates the robot. Only the joint angle offset errors are calculated here. The results of the joint angle offset error calculation are listed in Table 4.6. A discussion of these results is given in Section 6.2 .

Table 4.6: Joint angle offset determination for adjustment simulation.

\begin{tabular}{|l|l|l|l|}
\hline & Input $(\mathrm{deg})$ & Calculated $(\mathrm{deg})$ & Error $(\%)$ \\
\hline$\delta \theta_{2}$ & 0.35 & 0.3486 & -0.4094 \\
\hline$\delta \theta_{3}$ & 0.8 & 0.7988 & -0.1548 \\
\hline$\delta \theta_{4}$ & -0.7 & -0.7001 & 0.0212 \\
\hline$\delta \theta_{5}$ & 0.2 & 0.2011 & 0.5265 \\
\hline$\delta \theta_{6}$ & 0.1 & 0.1012 & 1.2178 \\
\hline
\end{tabular}




\section{Chapter 5}

\section{Experiment Method Refinement}

\subsection{Physical Setup and Experiment}

While the method described in Section 4.3 is functional in the simulated environment, some modifications were made to refine it to improve the precision for an actual physical experiment. These mainly include modifications to make image analysis more precise with the movement of the robot.

\subsubsection{Adjustment Process}

The experimental adjustment process is similar to the method described in Subsection 4.3 , which was applied to the simulation. The difference is the order in which each adjustment is performed.

The order of adjustment in the experiment is:

1. rotation adjustment about $\mathrm{X}$ axis (Subsection 4.3.3);

2. rotation adjustment about $\mathrm{Y}$ axis;

3. translation adjustment along $\mathrm{Z}$ axis (Subsection 4.3.2);

4. rotation adjustment about $\mathrm{Z}$ axis (Subsection 4.3.3);

5. translation adjustment along $\mathrm{X}$ axis (Subsection 4.3.2);

6. if measurements from cameras are within a specified threshold of 3 or 5 pixels (Subsection 5.1.2), accept the pose as correct. Otherwise, repeat this process. 
This change in order was made to make sure the laser was still reaching the cameras in approximately the centre of the camera sensor, as the image analysis is not capable of interpreting images in which the cross is near the edge of the image. The order of adjustments in the simulation (fully adjusting orientation before attempting any adjustment of position) was likely to move the cross to an un-readable edge of the image. In practice, by putting the Z-axis translation adjustment immediately after the $\mathrm{X}$-axis rotation adjustment, these would approximately counteract each other and return the cross to the centre of the image. The same is true of Z-axis rotation with $\mathrm{X}$-axis translation.

\subsubsection{Choosing Adjustment Threshold by Analyzing Re- peatability}

The requirements for choosing the threshold at which to consider a pose acceptable were chosen to be as small as possible while remaining feasible in the context of the experiment. In order to find the smallest feasible range of acceptable poses, an experiment was performed that analyses the overall repeatability of the robot, camera, and image processing. This experiment was performed simply by taking an image at a certain robot pose, moving the robot away from this pose and then back, and then taking another image. This was performed 10 times to generate an estimated point spread.

The results of this analysis are shown for Camera 1 in Figure 5.1 and for Camera 2 in Figure 5.2. In these figures, each circle represents a determined cross centre location. The average of all images is represented with an cross symbol. A circle is used to represent a distance of one half pixel from the average location.

It can be seen in Figures 5.1 and Figure 5.2 that Camera 1 (directly in line with the laser) has a substantially greater repeatability than Camera 2 (viewing the reflected light from the beam splitter). From this, the most restrictive threshold would seem to be 1 to 2 pixels when remaining at the same location. However, some inherent additional imaging inaccuracies do occur when moving back and forth along the line. Two different threshold levels were selected based on this: a high precision method using a 3 pixel threshold on each camera, and an average method using a 5 pixel threshold on each camera. In order for the high precision method to be successful, 


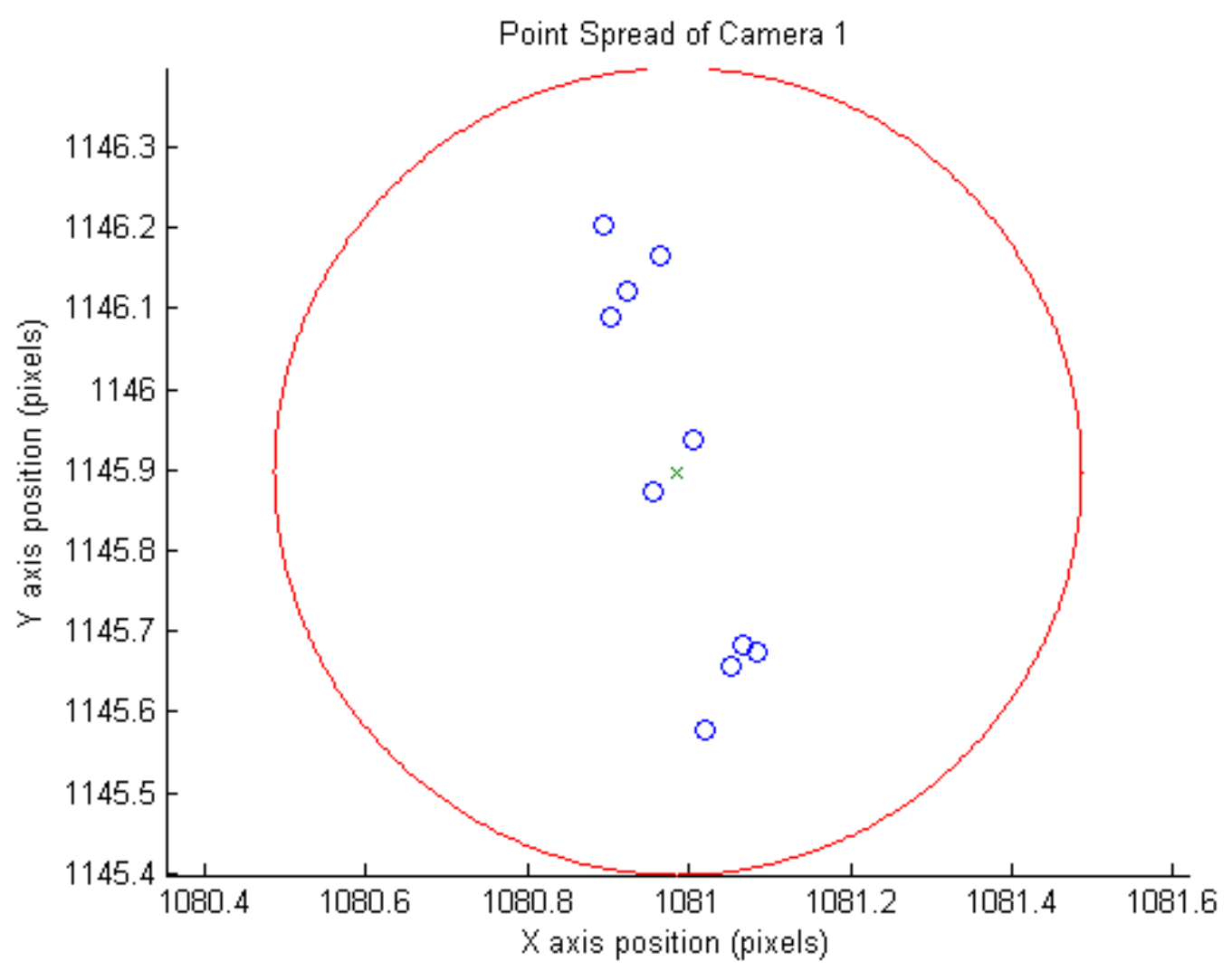

Figure 5.1: Point spread on Camera 1 of repeatability test. 


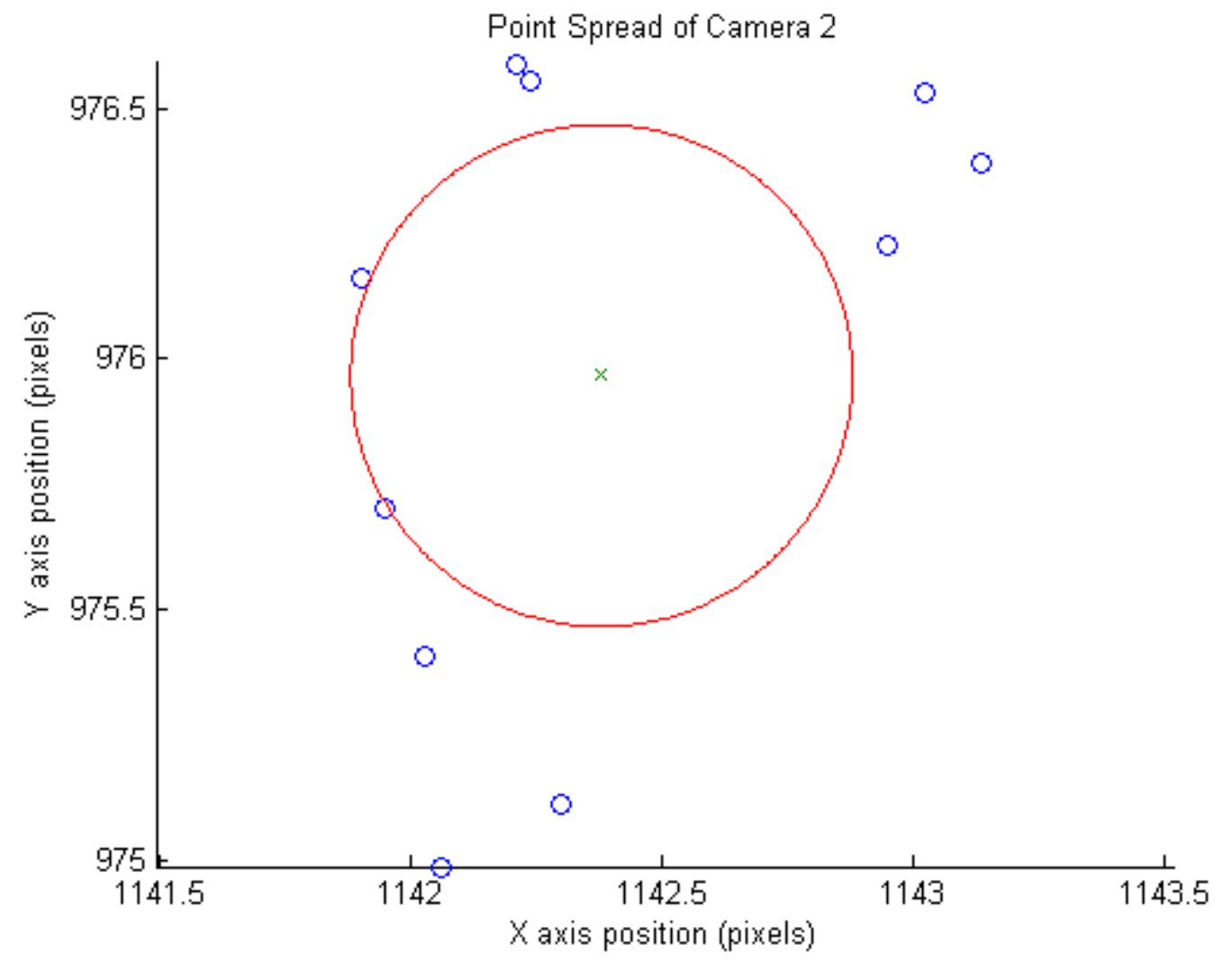

Figure 5.2: Point spread on Camera 2 of repeatability test. 
the overall time taken to perform a calibration increases (see Subsection 5.1.4), and an environment free from large magnitude vibrations is required.

\subsubsection{Backlash Adjustment}

Backlash, mentioned in Subsection 2.2.4, is difficult to model. In this case, no actual

modeling is done to predict the effect of backlash on the robot pose. Instead, the movement pattern of the robot is modified such that every pose is approached from the same rotational direction in each joint. The necessity of this modification was determined with a brief analysis, which showed that the direction of approach had a noticeable effect on the cross location in images, far more than could be explained by camera and image analysis error. When approaching a certain pose from a different direction, the cross centre location was up to 30 pixels away from the initial location. This is six times the threshold used to determine even a rough equivalence.

Each movement instruction sent to the robot was replaced with a three-step process. Starting from the desired pose in world frame coordinates, the following was performed.

1. A position was identified that was equivalent to a movement of a small amount (one quarter of a degree) in each joint, from the target pose. This is the "back" position from which the target pose will be approached.

2. The robot is instructed to move to this back position.

3. The robot is instructed to move to the target pose.

Using this method, each joint will always move in the same sense when approaching target poses.

\subsubsection{Calibration Time}

When all steps are considered, the calibration process becomes quite lengthy. Many factors contribute to this. The backlash adjustment in Subsection 5.1.3 modifies each movement to be two full movements. To enhance precision, the images were only taken a full second after the robot finished a motion. Because of the nature of the adjustments, for each individual adjustment, several movements and images were required, and several adjustments were required for each data point. Depending 
on the number of adjustments required before the imaging threshold was reached, a single data point could sometimes take up to 20 minutes.

In order to improve the calibration time, some modifications were made. The image threshold was set to be larger, as mentioned in Subsection 5.1.2. The total number of data points per set was between 10 and 15, unlike in the simulations in which 30 or more data points could be used. These resulted in a smaller data set, and lower precision in the pose measurement.

\subsubsection{Laser Offset Parameters}

The calibration routine relies on the laser line being oriented perfectly in the tool Y-axis. In practice, it is impossible to place the laser exactly along this line. In order to compensate for this, four additional unknown parameters are introduced that represent the differences in position and orientation between the actual laser axis and the tool Y-axis. The five-parameter optimization shown in Subsection 4.2.2 a nine-parameter optimization. The values determined for laser offset are shown in Subsections 6.3.1 and 6.3.2. 


\section{Chapter 6}

\section{Results}

\subsection{Simulation Results}

This section gives the results of a full simulated calibration to find the joint angle and link twist offsets. This is a two-stage process. The results of the initial determination of joint angle offsets are given in Table 6.1. The results of the modified determination of joint angle offsets are given in Table 6.2. The results of the determination of the link twist offsets are given in Table 6.3.

Table 6.1: Result of the initial determination of joint angle offsets.

\begin{tabular}{|r|r|r|r|}
\hline Joint & Identified angle (degrees) & Simulated angle (degrees) & Percent error \\
\hline 2 & 0.33 & 0.35 & -4.50 \\
\hline 3 & 0.84 & 0.80 & 5.42 \\
\hline 4 & -0.77 & -0.70 & 10.25 \\
\hline 5 & 0.16 & 0.20 & -21.77 \\
\hline 6 & 0.09 & 0.10 & -10.62 \\
\hline
\end{tabular}

\subsection{Discussion of Simulation Results}

The simulation process correctly determined joint angle offsets for Joints 4 and 5 with a high degree of precision. For these two joints, the determined values were 
Table 6.2: Result of the modified determination of joint angle offsets.

\begin{tabular}{|r|r|r|r|}
\hline Joint & Identified angle (degrees) & Simulated angle (degrees) & Percent error \\
\hline 2 & 0.33 & 0.35 & -4.50 \\
\hline 3 & 0.82 & 0.80 & 1.97 \\
\hline 4 & -0.70 & -0.70 & $4.43 \times 10^{-9}$ \\
\hline 5 & 0.20 & 0.20 & $-2.95 \times 10^{-8}$ \\
\hline 6 & 0.07 & 0.10 & -28.54 \\
\hline
\end{tabular}

Table 6.3: Result of the determination of link twist offsets.

\begin{tabular}{|r|r|r|l|}
\hline Joint & Identified angle (degrees) & Simulated angle (degrees) & Percent error \\
\hline 2 & 0.01 & 0.01 & $1.14 \times 10^{-7}$ \\
\hline 3 & 0.02 & 0.02 & $-9.36 \times 10^{-9}$ \\
\hline 4 & -0.01 & -0.01 & $-2.82 \times 10^{-8}$ \\
\hline 5 & 0.01 & 0.01 & $2.92 \times 10^{-7}$ \\
\hline 6 & -0.02 & -0.02 & $-1.03 \times 10^{-7}$ \\
\hline
\end{tabular}

within a very small fraction of a degree $\left(10^{-11}\right)$. The remaining joint angle offsets were determined with reasonable precision. The least accurate was Joint 6 , with $25 \%$ error, representing a difference of 0.03 degrees. The values of link twist offsets were determined with far greater precision in the simulation. This is likely due to the substantially lower values of these offsets. While simulated joint angle offsets ranged from 0.1 degrees to 0.8 degrees, the link twist offsets were all 0.02 degrees or smaller.

\subsection{Experimental Results}

This section gives the results of each experiment using both robots. All processed data sets are included, though not all data sets are considered in the analysis. The nature of the experiment is such that the data acquisition may occasionally fail completely. Vibration disturbance during image acquisition at any point in the calibration process may cause small errors, but if this vibration disturbance occurs during the acquisition of the nominal "target" image, the entire data set will be affected. These data sets are considered outliers and are excluded from the analysis. For each robot, outlying 
data sets will be identified.

\subsubsection{Experiment Using FANUC S-420iF Robot Arm}

A total of five complete data sets were collected with this robot. The results of these data sets are given in Tables 6.4 and 6.5 .

Table 6.4: Joint angle offset result of calibration process using FANUC s-420iF robot.

\begin{tabular}{|r|r|r|r|r|r|}
\hline & \multicolumn{5}{|c|}{ Joint angle offset (degrees) } \\
\hline Set & Joint 2 & Joint 3 & Joint 4 & Joint 5 & Joint 6 \\
\hline 1 & 0.13 & 0.09 & 0.20 & -0.01 & 45.41 \\
\hline 2 & 0.07 & 0.08 & 0.07 & 0.03 & 79.14 \\
\hline 3 & 6.69 & 9.04 & 23.48 & 10.76 & 158.13 \\
\hline 4 & 0.25 & 0.11 & -0.03 & 0.16 & 101.97 \\
\hline 5 & 0.18 & 0.12 & 0.18 & -0.01 & 45.41 \\
\hline
\end{tabular}

Table 6.5: Laser offset result of calibration process using FANUC s-420iF robot.

\begin{tabular}{|r|r|r|r|}
\hline \multicolumn{4}{|c|}{ Laser offset (degrees and mm) } \\
\hline X angle & Y angle & X offset & Y offset \\
\hline 0.01 & 44.87 & 0.00 & 0.00 \\
\hline 2.70 & 78.32 & 1.24 & -0.38 \\
\hline 31.02 & 96.73 & 0.94 & -0.09 \\
\hline 168.96 & 101.13 & -1.67 & -1.88 \\
\hline 0.01 & 44.87 & 0.00 & 0.00 \\
\hline
\end{tabular}

Of the identified joint angles, Joints 2 and 3 were identified with a lower variation in results. These two joints angles were analyzed to determine which data sets are excluded as outliers. Of these five sets, Data Set 3 was identified as an outlier. This is because the values identified for Joints 2 and 3 were over 3 standard deviations from the mean value given in Table 6.6. Excluding this outlier, the mean value 
of all data sets is presented in Table 6.6. Additionally, the standard deviation is presented to demonstrate how consistent the measurements are. While joints 2 and 3 are identified with consistent results, in joints 4,5 and 6 the standard deviation is larger than the identified joint offset. The values obtained for these joint angles are much less reliably identified. The standard deviations for the laser offset parameters are also high, although this is to be expected, as the tool holding the laser was frequently adjusted between gathering each data set.

Table 6.6: Average of results of calibration of FANUC s-420iF robot.

\begin{tabular}{|l|l|r|r|}
\hline & & Average & Standard deviation \\
\hline Joint Angle Offset (degrees) & Joint 2 & 0.16 & 0.07 \\
\hline & Joint 3 & 0.10 & 0.02 \\
\hline & Joint 4 & 0.10 & 0.11 \\
\hline & Joint 5 & 0.04 & 0.08 \\
\hline Laser Offset & Joint 6 & 67.98 & 27.68 \\
\hline (degrees and $\mathrm{mm})$ & X angle & 42.92 & 84.04 \\
\hline & Y angle & 67.30 & 27.52 \\
\hline & X offset & -0.11 & 1.19 \\
\hline & Y offset & -0.56 & 0.89 \\
\hline
\end{tabular}

\subsubsection{Experiment Using Motoman MH-180 Robot}

A total of eleven complete data sets were collected with this robot. The results of these data sets are given in Tables 6.7 and 6.8 .

Of these eleven sets, Data Sets 2, 5, 10, and 11 were identified as outliers. In this case, the values of joints 2 and 4 were considered to make this judgement. Excluding these outliers, the mean value of all data sets is presented in Table 6.9. Additionally, the standard deviation is presented to demonstrate how consistent the measurements are. 
Table 6.7: Joint angle result of calibration process using Motoman MH-180 robot.

\begin{tabular}{|r|r|r|r|r|r|}
\hline \multicolumn{5}{|c|}{ Joint angle offset (degrees) } \\
\hline Set & Joint 2 & Joint 3 & Joint 4 & Joint 5 & Joint 6 \\
\hline 1 & -0.03 & -0.31 & 0.15 & -0.39 & 63.93 \\
\hline 2 & -3.87 & -1.10 & -0.55 & -0.14 & 88.69 \\
\hline 3 & -0.02 & -0.34 & 0.17 & -0.42 & -32.55 \\
\hline 4 & -0.02 & -0.29 & 0.15 & -0.37 & 52.64 \\
\hline 5 & -2.56 & -3.26 & -5.25 & -1.86 & -11.26 \\
\hline 6 & -0.05 & -0.33 & 0.18 & -0.45 & -47.22 \\
\hline 7 & -0.02 & -0.29 & 0.15 & -0.37 & 52.64 \\
\hline 8 & -0.03 & -0.32 & 0.18 & -0.41 & 64.01 \\
\hline 9 & 0.08 & 1.28 & 0.18 & -1.25 & -90.36 \\
\hline 10 & -2.28 & 0.48 & 0.50 & -0.80 & 62.92 \\
\hline 11 & -4.81 & 12.95 & 5.22 & 11.85 & 268.95 \\
\hline
\end{tabular}

Table 6.8: Laser offset result of calibration process using Motoman MH-180 robot.

\begin{tabular}{|r|r|r|r|}
\hline \multicolumn{4}{|c|}{ Laser offset (degrees and mm) } \\
\hline X angle & Y angle & X offset & Y offset \\
\hline-0.56 & 64.15 & -0.01 & -0.21 \\
\hline 3.06 & 88.64 & -0.08 & -0.26 \\
\hline-0.20 & -32.28 & -0.04 & -0.19 \\
\hline-0.39 & 52.84 & -0.01 & -0.02 \\
\hline 1.30 & -10.90 & 0.00 & 0.00 \\
\hline-0.20 & -46.84 & -0.72 & -0.72 \\
\hline-0.39 & 52.84 & -0.01 & -0.02 \\
\hline-0.61 & 64.25 & 0.00 & -0.01 \\
\hline 0.01 & -90.16 & 0.00 & 0.00 \\
\hline 0.29 & 62.51 & -0.13 & 0.21 \\
\hline 0.07 & 4.89 & 0.16 & -0.24 \\
\hline
\end{tabular}


Table 6.9: Average of results of calibration of Motoman MH-180 robot.

\begin{tabular}{|l|l|r|r|}
\hline & & Average & Standard Deviation \\
\hline Joint Angle Offset (degrees) & Joint 2 & -0.01 & 0.04 \\
\hline & Joint 3 & -0.09 & 0.60 \\
\hline & Joint 4 & 0.16 & 0.02 \\
\hline & Joint 5 & -0.52 & 0.32 \\
\hline & Joint 6 & 9.01 & 64.05 \\
\hline Laser Offset & X angle & -0.34 & 0.22 \\
\hline (degrees and mm) & Y angle & 9.26 & 64.02 \\
\hline & X offset & -0.11 & 0.27 \\
\hline & Y offset & -0.17 & 0.26 \\
\hline
\end{tabular}

\subsubsection{Discussion of Experimental Results}

The precision of the calibration routine is determined here using consistency, because the actual joint offsets are not known. Multiple calibration routines are performed and the results are compared with each other. The standard deviation gives us a measure of the consistency of results.

The goal of the calibration procedure is to determine the value of several calibration parameters which remain constant throughout the process. Ideally, each calibration procedure would return the exact same result. However, in practice many different factors can affect the calibration procedure. The standard deviation is included with results to measure the degree to which the results remain consistent. A more consistent result will have a lower standard deviation. A consistent result indicates that the calibration procedure is able to successfully identify the parameter in question.

Some data sets obtained experimentally were deemed to be outliers. Certain factors can affect the precision of the data gathering method to the point that the analysis no longer produces reasonable results. These data sets are easily identifiable in the results because any small problem in data gathering tends to produce large errors in the analysis. Outliers were identified by examining the joint angles which had the most consistent results. The data gathered using the FANUC s-420iF gave reasonable results for some joint offsets, but not all. Consistent results were produced 
for Joint 4. Joints 2, 3, and 5 have reasonable results but the consistency is not high. The results for Joint 6 were not at all consistent or reasonable, but this may be due to the laser offset process in calibration, discussed further in Subsection 6.3.4. The results of the laser offset process were not consistent at all. However, this is to be expected because of changes in the laser's placement between gathering each data set. The data gathered using the Motoman MH-180 robot shows a similar lack of consistency in Joint 6 offsets, likely for the reason discussed in Subsection 6.3.4. The results were slightly less consistent than those from the FANUC s-420iF robot, but were larger overall. A larger offset is more difficult to correctly identify. The comparison may not be as apt here as a substantially larger number of data sets were obtained using the Motoman MH-180.

\subsubsection{Relationship Between Joint 6 Angle Offset and Laser Y Angle Offset}

The Joint 6 angle offset results were not consistent at all when compared with each other. However, the exact same inconsistency was seen in the results for the laser's Y angle offset. The difference between these two remained very consistent. An analysis of the difference between the Laser $\mathrm{Y}$ offset and Joint 6 is given for each robot, in Tables 6.10 and Table 6.11. The relatively high consistency of this difference implies that these two values are related. In fact, a each of these movements (Laser Y angle rotation and Joint 6 rotation) would move the laser in the same direction. Being able to accurately place the laser would aid in determining the actual Joint 6 offset. 
Table 6.10: Difference between Joint 6 angle offset and laser $\mathrm{Y}$ angle offset for FANUC s-420iF.

\begin{tabular}{|c|c|}
\hline Data Set & Difference \\
\hline 1 & 0.54 \\
\hline 2 & 0.82 \\
\hline 3 (outlier) & 61.40 \\
\hline 4 & 0.84 \\
\hline 5 & 0.54 \\
\hline Mean & 0.69 \\
\hline Standard Deviation & 0.17 \\
\hline
\end{tabular}

Table 6.11: Difference between joint 6 angle offset and laser $Y$ angle offset for Motoman MH-180.

\begin{tabular}{|c|c|}
\hline Data Set & Difference \\
\hline 1 & -0.22 \\
\hline 2 (outlier) & 0.05 \\
\hline 3 & -0.27 \\
\hline 4 & -0.20 \\
\hline 5 (outlier) & -0.36 \\
\hline 6 & -0.37 \\
\hline 7 & -0.20 \\
\hline 8 & -0.23 \\
\hline 9 & -0.21 \\
\hline 10 (outlier) & 0.41 \\
\hline 11 (outlier) & 264.06 \\
\hline Mean & -0.24 \\
\hline Standard Deviation & 0.06 \\
\hline
\end{tabular}




\section{Chapter 7}

\section{Concluding Remarks}

\subsection{Summary and Conclusions}

Chapter 1 provided an introduction to the use of robots in and the necessity of calibration. The motivation for this thesis was discussed: a novel method for calibration using simple, low-cost sensors. The main objectives of this thesis were listed and described. A statement of originality was included to identify the main original work described in this thesis.

Chapter 2 described the theoretical background needed for the calibration routine development. The mathematical representation of general robot motion was discussed, along with DH parameter conventions used in this thesis. A representative collection of calibration methods were summarized and discussed, including the methods from which the work in this thesis drew inspiration.

Chapter 3 described the experimental method. The physical experimental tools were discussed, including the cameras, robots, and the laser. The camera assembly was described, along with the mathematical interpretation of the camera images. The method of image analysis was fully disclosed.

Chapter 4 detailed the methods used for the simulation. The mathematical method used to perform the actual calibration once data is obtained was outlined. Two simulations were reported: a simple simulation was used to establish feasability wherein data sets were fully generated without considering robot motion, and a more realistic simulation in which the process of robot motion and image results were all considered.

Chapter 5 described modifications to Chapter 4s method that were applied to the experimental data collection. These included the sequence of movements used 
to collect a data point, an analysis of the repeatability of the imaging capture and analysis, modifications to avoid joint backlash, and a discussion of methods used to reduce the total time required for the calibration.

Chapter 6 provided the results of each calibration process. The simulation was able to identify 5 joint angle offsets and 5 link twist offsets. The experimental results identified 2 joint angle offsets with reasonable confidence and 2 joint angle offsets with lower confidence.

In this thesis, a method for calibration of six axis serial wrist partitioned robots was discussed, simulated, and implemented experimentally. Calibration was performed by constraining the end-effector motion to a single line in space and retaining constant end-effector orientation. This allows the calibration routine to be performed with simple and low cost sensing equipment. The linear constraint was enforced using a laser diode mounted to the robot end-effector, with an optical attachment producing a cross shape. This created a "virtual closed kinematic chain," with the laser line considered an additional link to ground, constraining the robot motion.

The simulated calibration was able to identify 10 of the 12 angular DH parameters (joint angle offsets and link twist errors). The experimental calibration routine was able to identify 5 of the 6 joint angle offsets, though only 2 were identified with a measure of statistical confidence. The reasonable precision of the simulation implies that this method is viable. The experimental precision was significantly less, and as such the link twist offsets were not able to be identified. This is because the identification of the link twist errors depends on the precision of the identified joint angle offsets. As joint angle offsets are much larger than link twist errors, the influence of the joint angle offsets that were not precisely identified will affect the link twist error identification and any results will simply be a reflection of this remaining joint angle error.

\subsection{Recommendations}

The experimental procedure should be redesigned to improve precision and confidence level, which might then lead to experimental identification of the link twist offsets. In order to increase the experimental precision and confidence level:

1. The experiment should be performed in an environment free from machine vibration. This experiment was performed on a factory floor during operating 
hours. While every effort was made to time the experiment to avoid disturbing vibrations, this was not fully possible. In a calm environment the imaging would be substantially more accurate. The major source of vibration during this experiment was a sheet metal punch press frequently running, only a few metres from the experiment. Additionally, a press brake and a welding robot were both operating very close by.

2. Environmental factors affecting the optical equipment, such as dust and ambient light, could be reduced. The experiment operated in an incredibly dusty environment. The cameras and beam splitter were contained in a metal enclosure to keep out some dust and ambient light, but the window leading into this enclosure had to be frequently cleaned and a layer of dust can have substantial negative effects on camera sensing.

3. The process could be modified to run autonomously, and therefore run overnight without oversight. This would allow a very long calibration procedure to run.

4. A different imaging method or sensor might provide for increased accuracy. In this experiment, cameras were chosen in order to view a laser cross rather than a laser point. However, these cameras provide decreased accuracy when compared with the PSDs used in other experiments reported in the literature.

Additionally, a method used to estimate errors in the remaining DH paramenter offsets: link length $(a)$ and link offset $(d)$ was attempted but was not included in this thesis, as simulated results were not obtained successfully. This method is described in Appendix A. If the precision of the measurement acquisition and analysis were substantially increased, this method to obtain the remaining DH parameter offsets might be viable. 


\section{List of References}

[1] J. Ruurda, T. J. van Vroonhoven, and I. Broeders, "Robot-assisted surgical systems: a new era in laparoscopic surgery.," Annals of the Royal College of Surgeons of England, vol. 84, no. 4, p. 223, 2002.

[2] Y. S. Kwoh, J. Hou, E. Jonckheere, S. Hayati, et al., "A robot with improved absolute positioning accuracy for ct guided stereotactic brain surgery," Biomedical Engineering, IEEE Transactions on, vol. 35, no. 2, pp. 153-160, 1988.

[3] J. J. Craig, Introduction to Robotics. Pearson ; Upper Saddle River, NJ, 2005.

[4] P. Shiakolas, K. Conrad, and T. Yih, "On the accuracy, repeatability, and degree of influence of kinematics parameters for industrial robots," International journal of modelling and simulation, vol. 22, no. 4, pp. 245-254, 2002.

[5] Kuka, "Kuka KR 15/2 specification." Datasheet, 2011.

[6] ABB, "IRB 120 industrial robot." http://new.abb.com/products/robotics/ industrial-robots/irb-120, 2012.

[7] R. P. Judd and A. B. Knasinski, "A technique to calibrate industrial robots with experimental verification," Robotics and Automation, IEEE Transactions on, vol. 6, no. 1, pp. 20-30, 1990.

[8] M. Leitner, M. Hayes, R. Ofner, C. Sallinger, and P. OLeary, "Thermal effects and consequences for repeatability of an industrial robot," in Proc. 18th Canadian Congress of Applied Mechanics, St. Johns, Canada, June, 2001.

[9] B. Du, N. Xi, and E. Nieves, "Industrial robot calibration using a virtual linear constraint," International Journal on Smart Sensing and Intelligent Systems, vol. 5, no. 4, pp. 987-1000, 2012.

[10] J. P. Merlet, Parallel Robots. Dordrecht ; Boston, MA : Kluwer Academic Publishers, 2006.

[11] D. Stewart, "A platform with six degrees of freedom," in Proc. Inst. Mech. Eng. London, 1965.

[12] V. Gough, "Contribution to discussion of papers on research in automobile stability, control and tyre performance," in Proc. Auto Div. Inst. Mech. Eng, 1956.

[13] S. Briot and I. Bonev, "Are parallel robots more accurate than serial robots?," CSME Transactions, vol. 31, no. 4, pp. 445-456, 2007. 
[14] FANUC Robotics, "S-420i." Datasheet, 1998.

[15] Yaskawa Motoman Robotics, "MH180 MH225." http://www.motoman.com/ datasheets/MH180_MH225.pdf, 2014.

[16] T. K. Tanev, "Kinematics of a hybrid (parallel-serial) robot manipulator," Mechanism and Machine Theory, vol. 35, no. 9, pp. 1183-1196, 2000.

[17] L. Romdhane, "Design and analysis of a hybrid serial-parallel manipulator," Mechanism and Machine Theory, vol. 34, no. 7, pp. 1037-1055, 1999.

[18] J. D. Robinson and M. J. D. Hayes, "The dynamics of a single algebraic screw pair," Transactions of the Canadian Society for Mechanical Engineering, vol. 35, no. 4, pp. 491-504, 2011.

[19] R. Beardmore, "Simple linkages." http://www.roymech.co.uk/Useful Tables/Mechanics/Linkages.html, 2011. [Online; accessed 20-November-2015].

[20] J. Denavit and R. S. Hartenberg, "A kinematic notation for lower-pair mechanisms based on matrices," Trans. ASME, Journal of Applied Mechanics, vol. 22, pp. 215-221, June 1955.

[21] H. Lipkin, "A note on denavit-hartenberg notation in robotics," in Proceedings of IDETC/CIE, 2005.

[22] D. Kohli and M. Osvatic, "Inverse kinematics of general 6R and 5R, P serial manipulators," Journal of Mechanical Design, vol. 115, no. 4, pp. 922-931, 1993.

[23] D. Manocha and J. F. Canny, "Efficient inverse kinematics for general 6R manipulators," Robotics and Automation, IEEE Transactions on, vol. 10, no. 5, pp. 648-657, 1994.

[24] D. L. Pieper, "The kinematics of manipulators under computer control," tech. rep., DTIC Document, 1968.

[25] Z. Roth, B. Mooring, and B. Ravani, "An overview of robot calibration," IEEE Journal on Robotics and Automation, vol. 5, no. 3, pp. 377-385, 1987.

[26] C. Gong, J. Yuan, and J. Ni, "Nongeometric error identification and compensation for robotic system by inverse calibration," International Journal of Machine Tools and Manufacture, vol. 40, no. 14, pp. 2119-2137, 2000.

[27] A. Zalucky and D. Hardt, "Active control of robot structure deflections," Journal of dynamic systems, measurement, and control, vol. 106, no. 1, pp. 63-69, 1984.

[28] M. Ruderman, F. Hoffmann, and T. Bertram, "Modeling and identification of elastic robot joints with hysteresis and backlash," Industrial Electronics, IEEE Transactions on, vol. 56, no. 10, pp. 3840-3847, 2009.

[29] S. Takata, A. Yamada, T. Kohda, and H. Asama, "Life cycle simulation applied to a robot manipulator-an example of aging simulation of manufacturing facilities," CIRP Annals-Manufacturing Technology, vol. 47, no. 1, pp. 397-400, 1998. 
[30] A. Elatta, L. P. Gen, F. L. Zhi, Y. Daoyuan, and L. Fei, "An overview of robot calibration," Information Technology Journal, vol. 3, no. 1, pp. 74-78, 2004.

[31] FANUC, "FANUC robot ARC mate 100i model B and M-6i model B maintenance manual," 2008.

[32] M. R. Driels, L. W. Swayze, and L. S. Potter, "Full-pose calibration of a robot manipulator using a coordinate-measuring machine," The International Journal of Advanced Manufacturing Technology, vol. 8, no. 1, pp. 34-41, 1993.

[33] H. Zhuang, K. Wang, and Z. S. Roth, "Simultaneous calibration of a robot and a hand-mounted camera," IEEE Transactions on Robotics and Automation, vol. 11, no. 5, pp. 649-660, 1995.

[34] C.-C. D. Lu and M. J. D. Hayes, "Kinematic calibration of 6 R serial manipulators using relative measurements," in CCToMM Mechanisms, Machines, and Mechatronics (M3) Symposium, 2013.

[35] H. W. Stone and A. C. Sanderson, "A prototype arm signature identification system," in Robotics and Automation. Proceedings. 1987 IEEE International Conference on, vol. 4, pp. 175-182, IEEE, 1987.

[36] N. Simpson and M. J. D. Hayes, "Simulation of a kinematic calibration procedure that employs the relative measurement concept," in Proc. CSME Forum, 2004.

[37] K. English, M. J. D. Hayes, M. Leitner, and C. Sallinger, "Kinematic calibration of six-axis robots," in Proceedings of the CSME Forum, Kingston (Canada), 2002.

[38] I.-C. Ha, "Kinematic parameter calibration method for industrial robot manipulator using the relative position," Journal of mechanical science and technology, vol. 22 , no. 6 , pp. 1084-1090, 2008.

[39] M. J. D. Hayes and P. O'Leary, "Kinematic calibration procedure for serial robots with six revolute axes," Technical Report, Institute for Automation, University of Leoben, 2001.

[40] W. H. Press, S. A. Teukolsky, W. T. Vetterling, and B. P. Flannery, Numerical recipes in $C$, vol. 2. Citeseer, 1996.

[41] F. Deumlich, Surveying Instruments. W. de Gruyter, 1982.

[42] M. R. Driels and U. S. Pathre, "Robot calibration using an automatic theodolite," The International Journal of Advanced Manufacturing Technology, vol. 9, no. 2, pp. 114-125, 1994.

[43] M. R. Driels and U. S. Pathre, "Vision-based automatic theodolite for robot calibration," Robotics and Automation, IEEE Transactions on, vol. 7, no. 3, pp. 351-360, 1991.

[44] J. F. Jarvis, "Microsurveying: towards robot accuracy," in Robotics and Automation. Proceedings. 1987 IEEE International Conference on, vol. 4, pp. 1660-1665, IEEE, 1987. 
[45] K. Lau, R. Hocken, and L. Haynes, "Robot performance measurements using automatic laser tracking techniques," Robotics and computer-integrated manufacturing, vol. 2, no. 3, pp. 227-236, 1985.

[46] D. J. Bennett and J. M. Hollerbach, "Autonomous calibration of single-loop closed kinematic chains formed by manipulators with passive endpoint constraints," Robotics and Automation, IEEE Transactions on, vol. 7, no. 5, pp. 597606, 1991.

[47] A. Omodei, G. Legnani, and R. Adamini, "Calibration of a measuring robot: Experimental results on a 5 dof structure," Journal of Robotic Systems, vol. 18, no. 5, pp. 237-250, 2001.

[48] L. P. Foulloy and R. B. Kelley, "Improving the precision of a robot," in Robotics and Automation. Proceedings. 1984 IEEE International Conference on, vol. 1, pp. 62-67, IEEE, 1984.

[49] A. Nubiola and I. A. Bonev, "Absolute robot calibration with a single telescoping ballbar," Precision Engineering, vol. 38, no. 3, pp. 472-480, 2014.

[50] M. Ikits and J. M. Hollerbach, "Kinematic calibration using a plane constraint," in Robotics and Automation, 1997. Proceedings., 1997 IEEE International Conference on, vol. 4, pp. 3191-3196, IEEE, 1997.

[51] H. Zhuang, S. H. Motaghedi, and Z. S. Roth, "Robot calibration with planar constraints," in Robotics and Automation, 1999. Proceedings. 1999 IEEE International Conference on, vol. 1, pp. 805-810, IEEE, 1999.

[52] M. A. Meggiolaro, G. Scriffignano, and S. Dubowsky, "Manipulator calibration using a single endpoint contact constraint," in Proceedings of ASME Design Engineering Technical Conference, Baltimore, USA, 2000.

[53] J. M. Hollerbach, A. Nahvi, and V. Hayward, "Calibration of a parallel robot using multiple kinematic closed loops," Citeseer, 1994.

[54] C. S. Gatla, R. Lumia, J. Wood, and G. Starr, "An automated method to calibrate industrial robots using a virtual closed kinematic chain," Robotics, IEEE Transactions on, vol. 23, no. 6, pp. 1105-1116, 2007.

[55] C. Wang, W. Chen, and M. Tomizuka, "Robot end-effector sensing with position sensitive detector and inertial sensors," in Robotics and Automation (ICRA), 2012 IEEE International Conference on, pp. 5252-5257, IEEE, 2012.

[56] Edmund Optics, EO-5012 Monochrome USB 3.0 Camera, 2014.

[57] D. Litwiller, "Ccd vs. cmos," Photonics Spectra, vol. 35, no. 1, pp. 154-158, 2001.

[58] H. P. VC, "Method and means for recognizing complex patterns," Dec. 181962. US Patent 3,069,654.

[59] J. Illingworth and J. Kittler, "A survey of the hough transform," Computer vision, graphics, and image processing, vol. 44, no. 1, pp. 87-116, 1988. 
[60] R. O. Duda and P. E. Hart, "Use of the hough transformation to detect lines and curves in pictures," Communications of the $A C M$, vol. 15, no. 1, pp. 11-15, 1972.

[61] D. H. Ballard, "Generalizing the Hough transform to detect arbitrary shapes," Pattern recognition, vol. 13, no. 2, pp. 111-122, 1981.

[62] R. Ofner, P. O'Leary, and M. Leitner, "A collection of algorithms for the determination of construction points in the measurement of $3 \mathrm{~d}$ geometries via light-sectioning," in 2nd Workshop on European Scientific and Industrial Collaboration Promoting: Advanced Technologies in Manufacturing, pp. 505-512, 1999 


\section{Appendix A}

\section{Possible Method for Determining Error in Link Length and Link Offset}

The following method was considered to determine errors in link length and link offset using a simple distance measurement on the end effector. This method requires very precise determination of the joint angle and link twist parameters. The joint angle and link twist parameters determined in this thesis were not precise enough to allow this method to work. However, if a higher precision results were obtained, this method may become viable.

Precise estimates of the joint offsets for all angular DH parameters allows a very accurate estimation of the end effector orientation at any given position of the robot. Using measurements of the distance between points along the line, and the known line orientation, these distance measurements are converted into measurements of relative position in the $\mathrm{X}, \mathrm{Y}$, and $\mathrm{Z}$ directions.

Following this, the relative measurement technique described in [34] can be used to determine the other DH parameter offsets.

This is done by solving:

$$
\Delta x=J_{r e l} \Delta \zeta
$$

Where $J_{r e l}$ is the relative Jacobian, and $\Delta x$ is the measure of relative position and orientation. For each point other than point $1, \Delta x$ is the relative $\mathrm{x}, \mathrm{y}$, and $\mathrm{z}$ positions calculated earlier, and the known orientation changes, which remain zero as 
the orientation is known to remain constant:

$$
\Delta x_{i}=\left[\begin{array}{c}
\Delta x_{i} \\
\Delta y_{i} \\
\Delta z_{i} \\
0 \\
0 \\
0
\end{array}\right] .
$$

At this point, the process is identical to that described in Subsection 2.3.4. 


\section{Appendix B}

\section{MATLAB Code used}

This appendix provides the code used to obtain and analyze data sets. The code written simply to communicate and provide commands to the robot is omitted here.

\section{B.0.1 Data Analysis}

\%This function puts all experimental data into variable "ThController": ThController $=$ runset 1 ;

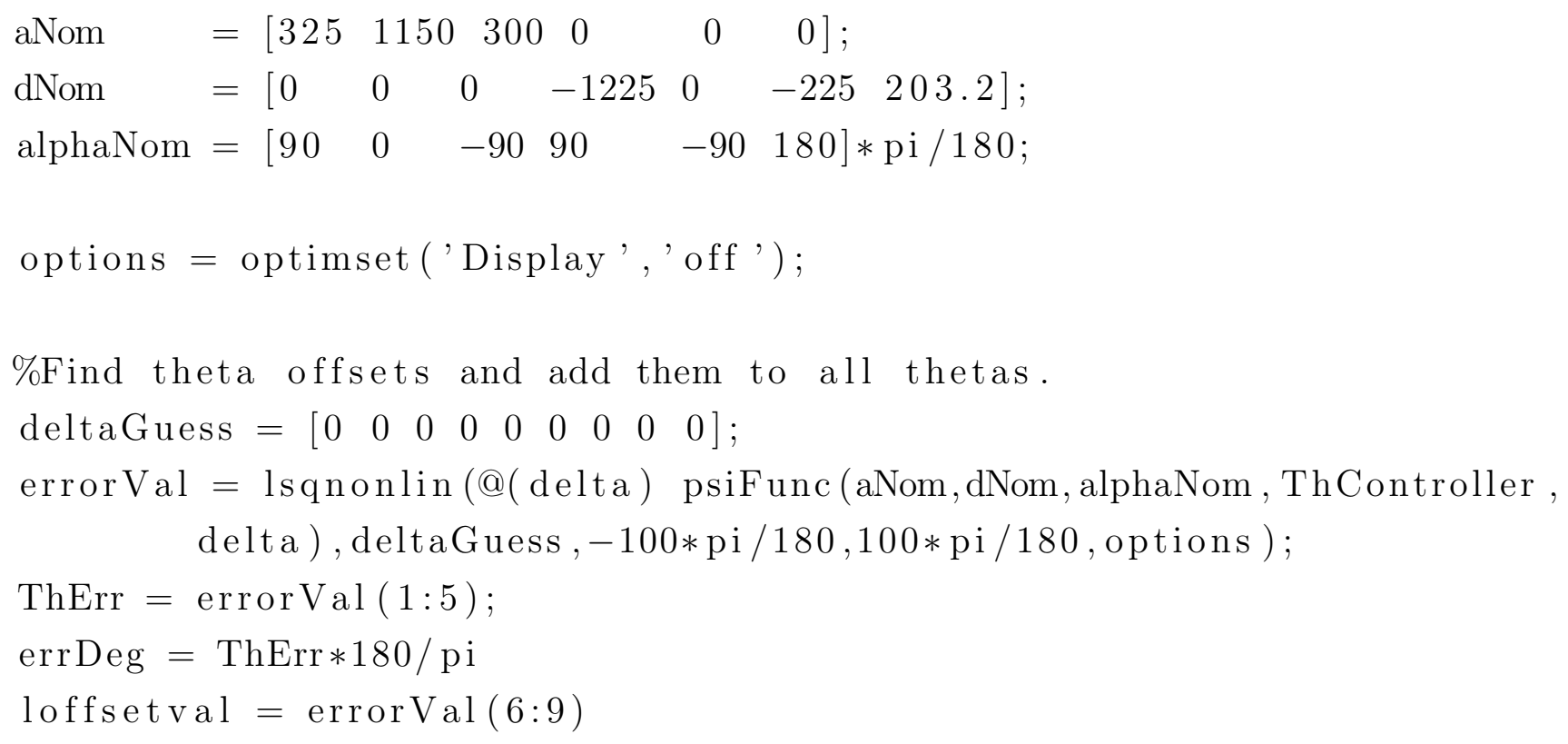




\section{B.0.2 Main Adjustment Process to Determine Data}

This process depends on separate scripts which adjust in transation or rotation, referred to as "MovAdjust" and "RotAdjust" respecitvely, along with the axis in which the adjustment is to take place.

$\%$ Parameters :

numPoses $=15$;

endPose $=[1312.813$

495.623

$-0.56834727$

$-0.13706071$

$0.029619983]$

startPose $=\operatorname{getPos}(\mathrm{t})$;

distthresh $=5$;

angthresh $=0.1 * \mathrm{pi} / 180$;

$\%$ Recording:

recidjnt $=$ fopen(' adjustrecordjnt.txt', 'w');

$\% \%$ Wind back and return to first point:

movetoWorldFB(t, startPose); pause (1);

$\%$ Nominal camera position at first pose:

$\mathrm{I}=\operatorname{getcam}(\operatorname{cam} 1, \operatorname{img} 1) ; \mathrm{J}=\operatorname{getcam}(\operatorname{cam} 2, \operatorname{img} 2) ; \mathrm{J}=\mathrm{fliplr}(\mathrm{J})$;

nomcam1 $=\operatorname{imAn}(\mathrm{I}) ; \operatorname{nomcam} 2=\operatorname{imAn}(\mathrm{J})$;

startJoint $=$ getJoint $(t)$;

fprintf(recidjnt,' -- HOME - \%15.8f \%15.8f \%15.8f \%15.8f \%15.8f $\% 15.8 \mathrm{f} \backslash \mathrm{r} \backslash \mathrm{n}^{\prime}$, startJoint );

$\%$ Go through next poses:

figure ;

for $\mathrm{i}=1$ : numPoses

startpointPose $=\operatorname{getPos}(\mathrm{t})$;

$\%$ Next pose as tool frame movement:

moveTool (t, $\left.\left[\begin{array}{llllll}0 & -50 & 0 & 0 & 0 & 0\end{array}\right], 2\right)$ 


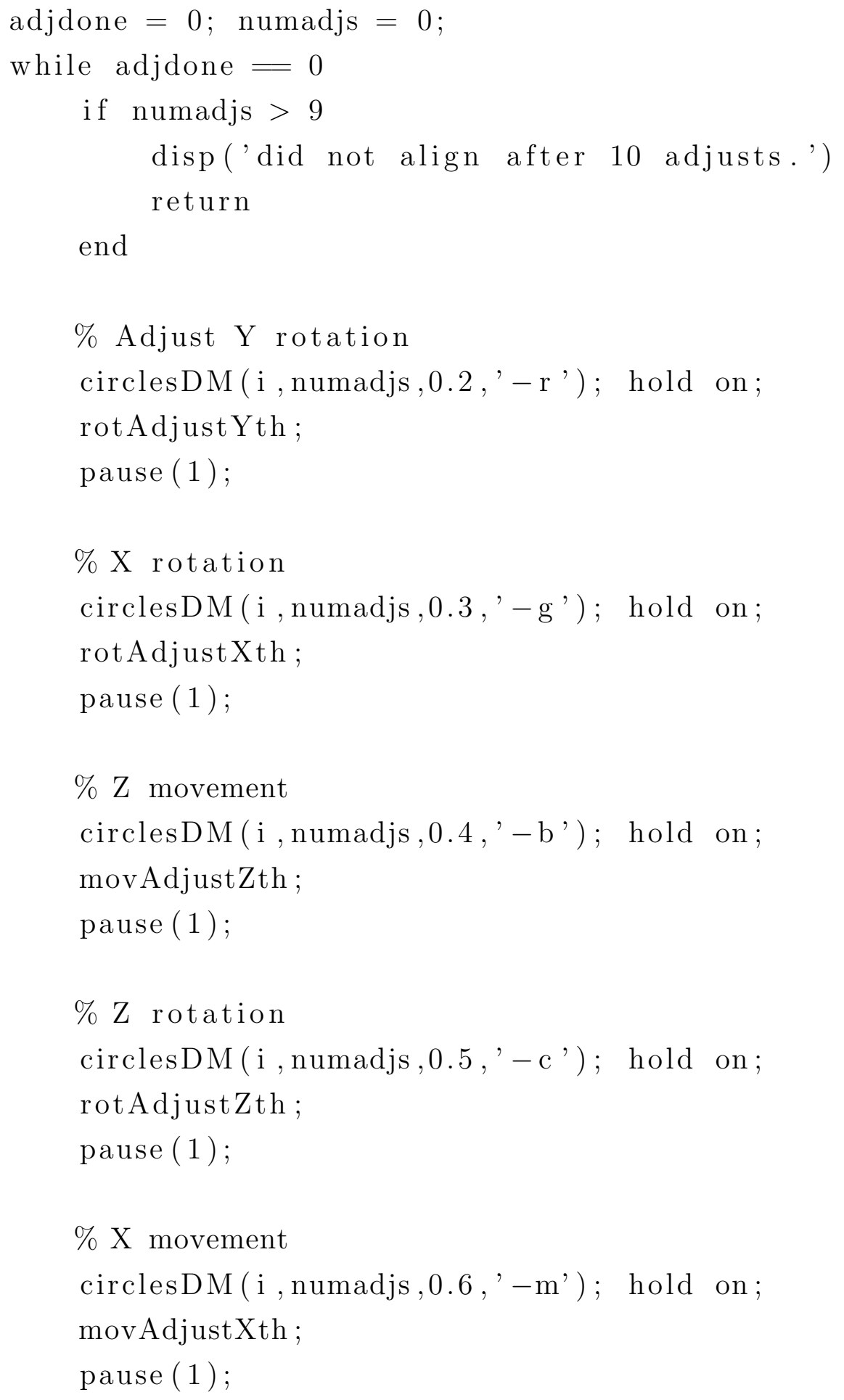




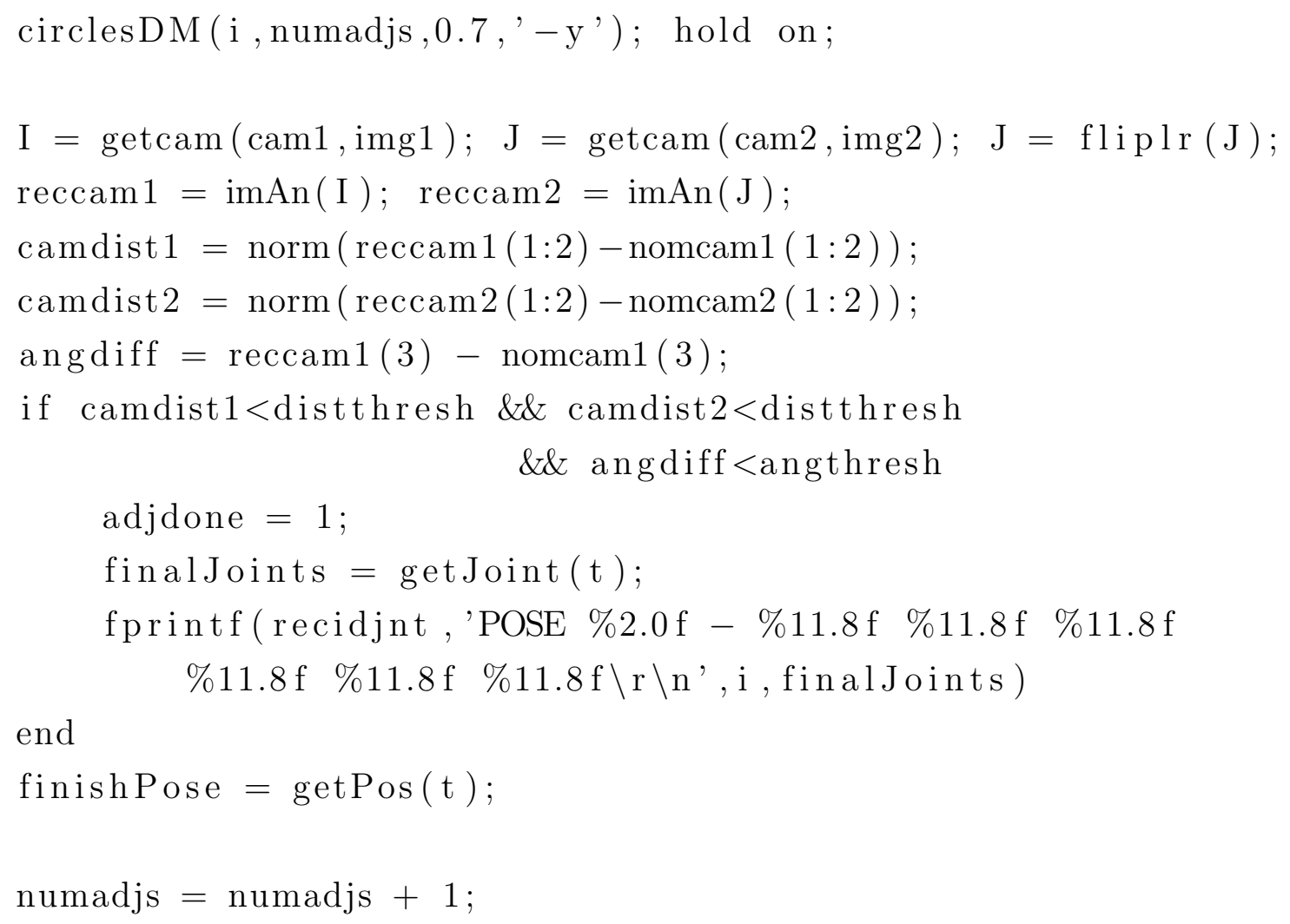

end

finishPose $=\operatorname{getPos}(\mathrm{t})$;

numadjs $=$ numadjs +1

end

end

fclose (recidjnt);

\section{B.0.3 Rotation Adjustment Code (Y Rotation)}

pause(1); \% Pause sometimes necessary if robot stops very suddenly. $\mathrm{I}=\operatorname{getcam}(\operatorname{cam} 1, \operatorname{img} 1)$;

vcam1 $=\operatorname{imAn}(\mathrm{I})$;

$\%$ Get angle:

$\operatorname{angcam} 1=\operatorname{vcam} 1(3)$;

angnomcam1 $=$ nomcam1 $(3)$; 
\%\% Step 2: Calculate angle difference.

movAmt $=-($ angnomcam $1-$ angcam 1$)$;

$\%$ Step 2: Move by difference

$\%$ (\& compensate for dist between laser and $\mathrm{x}$ )

moveTool(t, $\left.\left[\begin{array}{llllll}0 & 0 & 0 & 0 & \text { movAmt } & 0\end{array}\right], 2\right)$;

ldist $=188.2$;

\section{B.0.4 Translation Adjustment Code (Z Translation)}

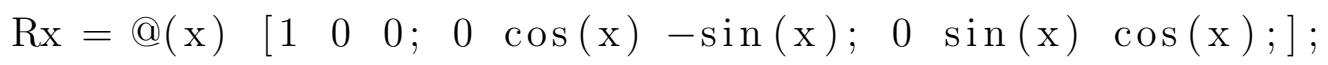

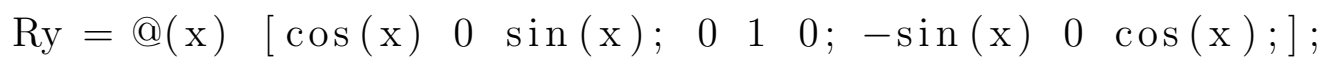

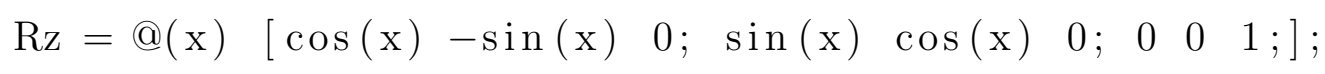

movAmt $=2$; \%how much to move by for test.

startadjPos $=\operatorname{getPos}(t)$;

pause(1); \% Pause sometimes necessary if robot stops very suddenly.

$\mathrm{I}=\operatorname{getcam}(\operatorname{cam} 1, \operatorname{img} 1)$;

fcam $1=\operatorname{imAn}(\mathrm{I})$;

camDiff1 = fcam1 - nomcam1;

$\%$ Move by amount:

moveTool(t, $\left.\left[\begin{array}{llllll}0 & 0 & \text { movAmt } & 0 & 0 & 0\end{array}\right], 2\right)$;

\% Figure out which position along this line is best, record:

pause(1); \% Pause sometimes necessary if robot stops very suddenly.

$\mathrm{I}=\operatorname{getcam}(\operatorname{cam} 1, \operatorname{img} 1)$;

$\% \%$ If this movement is off the grid, do the same in the other direction clear err;

try

$$
\text { fcam } 1=\operatorname{imAn}(I)
$$




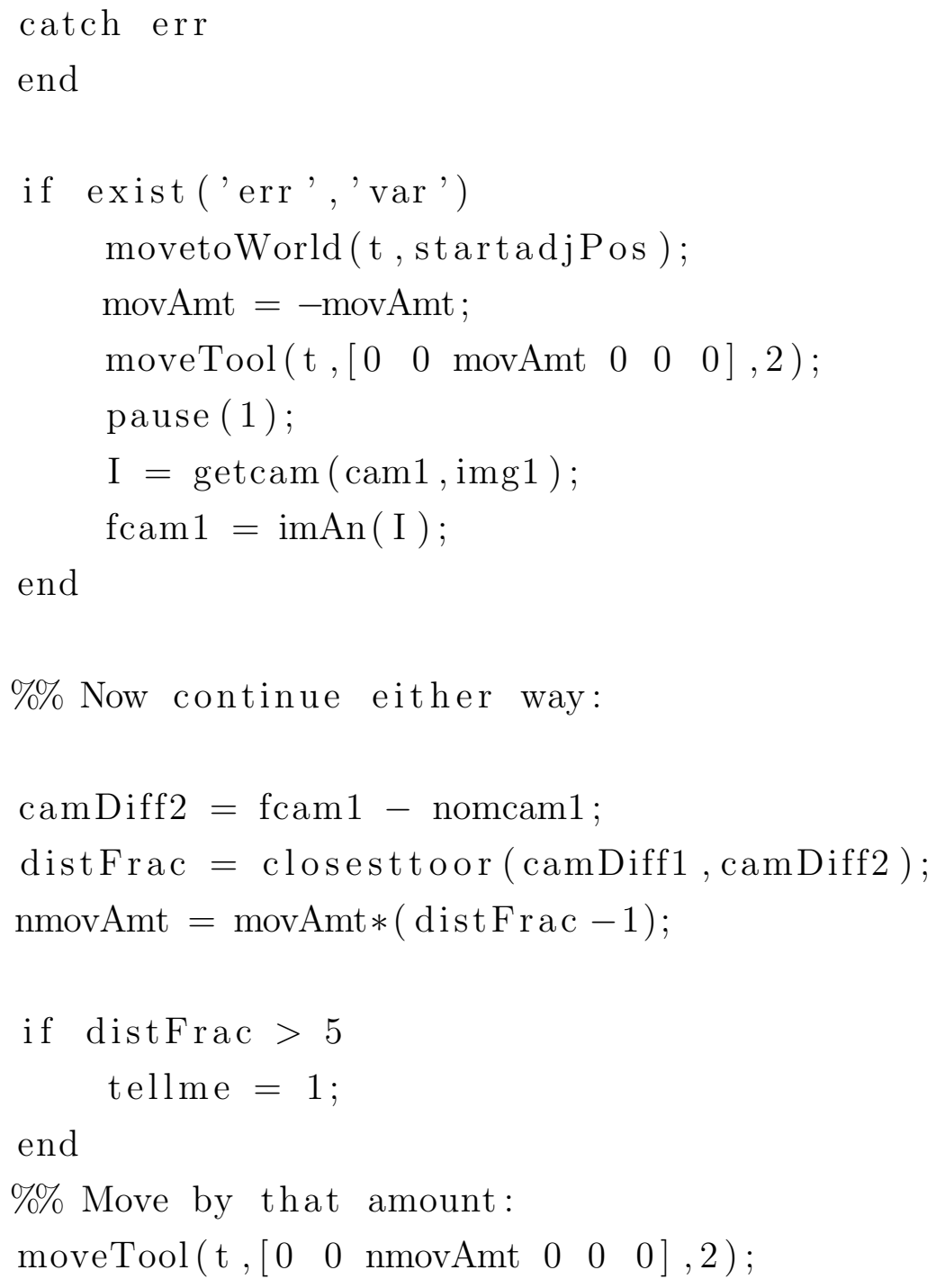

